\title{
Predicción de coeficientes efectivos de dispersión y difusión en medios porosos
}

Helen Denise Lugo Méndez 



\section{Universidad Autónoma Metropolitana}

\section{Iztapalapa}

Casa abierta al tiempo

Predicción de coeficientes efectivos de dispersión y difusión en medios porosos

TESIS PARA OBTENER EL GRADO DE MAESTRO EN CIENCIAS (INGENIERÍA QUIMICA)

Presenta: I. Q. Helen D. Lugo Méndez Asesor: Dr. J. Alberto Ochoa Tapia 



\section{Agradecimientos}

En primer lugar agradezco al Consejo Nacional de Ciencia y Tecnología (CONACyT) por su apoyo y patrocinio para la realización de este trabajo de investigación.

Al Dr. Jesús Alberto Ocho Tapia, mi asesor de tesis, por su confianza, orientación y paciencia para que esta tesis llegara a buen término.

Muy especialmente al Dr. Francisco J. Valdés Parada por su invaluable apoyo, interés, tiempo y motivación que me ha brindado en la realización de este proyecto.

Al Dr. Mario Vizcarra Mendoza por su confianza y apoyo para poder realizar el estudio de la Maestría en Ciencias en la Universidad Autónoma Metropolitana, Iztapalapa.

A los Doctores J. Obet Marroquín de la Rosa, Hugo Jiménez Islas, Gilberto Espinosa Paredes, por sus valiosas observaciones para mejorar el trabajo.

A mis papás y a mi hermano por su apoyo incondicional, por su amor, comprensión y motivación en todo momento. A mis abuelos, tíos y primos por apoyarme siempre.

A Gustavo por amarme y motivarme a superarme siempre y finalmente un infinito agradecimiento a mi hija Sabina que en su amor y sonrisa encontré las fuerzas necesarias para llegar hasta el final. 


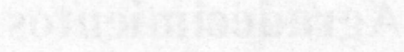




\section{Resumen}

En la ecuación de medio efectivo para el proceso de dispersión pasiva en medios porosos homogéneos, contiene un tensor efectivo de dispersión total. De acuerdo al método del promedio volumétrico, las componentes del tensor efectivo de dispersión se predicen a partir del problema de cerradura, definido en una celda periódica. Una simplificación de la celda periódica representativa del medio poroso espacialmente periódico, es una celda unitaria en la que su frontera externa presenta la misma geometría que la interfase, esta celda es llamada celda de Chang. En este trabajo, se usa la teoría de las funciones de Green para encontrar la solución analítica al problema de cerradura y el método de deferencias finitas ADI (Alternating Direction Implicit Method) para obtener la solución numérica a dicho problema, en la celda de Chang con geometría esférica y para las componentes del campo de velocidad se usan las expresiones algebraicas obtenidas bajo la condición de flujo potencial. Los valores de los coeficientes efectivos de transporte obtenidos se compararan con resultados teóricos y experimentales previos publicados en la literatura. $\mathrm{La}$ comparación es muy buena para números de Péclet menores que $10^{3}$, y para valores hasta $10^{5}$ la tendencia es análoga. 



\section{CONTENIDO}

1 Antecedentes 4

1.1 Dispersión 4

1.2 Algunos resultados teóricos y experimentales $\quad 7$

2 Problema de investigación 9

2.1 Objetivos del problema 9

$\begin{array}{ll}2.2 \text { Justificación del problema } & 10\end{array}$

3 Ecuación y coeficientes de medio efectivo del proceso de dispersión $\begin{array}{ll}\text { pasiva } & 11\end{array}$

3.1 Ecuaciones espacio-temporales y de medio efectivo del proceso de dispersión pasiva

3.2 Adimensionalización

4 Solución analítica al problema de cerradura en la celda unitaria de Chang

4.1 La celda de Chang y el problema de cerradura 22

4.2 Solución al problema de cerradura usando la función de Green 24

4.3 Equivalencia del problema de cerradura con la ecuación de Poisson en la celda de Chang

4.4 Unicidad de los problemas equivalentes en la celda de Chang 35

4.4.1 Problema de cerradura 35

$\begin{array}{lll}\text { 4.4.2 Ecuación de Poisson } & 37\end{array}$

4.5 Coeficientes de dispersión total 38

4.6 Función de Green y coeficiente de dispersión total en la celda de Chang con geometría esférica $\quad 39$

4.6.1 Celda de Chang con geometría esférica 39

4.6.2 Solución a la ecuación de Poisson con condiciones de frontera no-homogéneas 
usando la función de Green en la celda de Chang con geometría esférica

4.7 Coeficiente de dispersión total en la celda esférica de Chang

5 Condiciones de flujo y perfil de velocidad

5.1 Flujo reptante y perfil de velocidad alrededor de una esfera bajo condiciones de flujo reptante

5.2 Flujo potencial (no-viscoso) y perfil de velocidad alrededor de una esfera bajo condiciones de flujo potencial

5.3 Campo de velocidad, campo de velocidad promedio y campo de desviación espacial de la velocidad adimensionales

5.3.1 Flujo reptante

5.3.2 Flujo potencial

6 Solución numérica al problema de cerradura en la celda de Chang con geometría esférica

6.1 Diferencias finitas

6.2 ADI-2D

6.3 Algoritmo numérico

7 Resultados

7.1 Coeficiente longitudinal de dispersión total

7.2 Coeficiente transversal de dispersión total

8 Conclusiones

Nomenclatura

Bibliografía

Apéndices

A.1 Perfil de velocidad alrededor de una esfera de radio $\boldsymbol{R}$ bajo condiciones de flujo reptante

A.2 Solución a la ecuación de Poisson con condiciones de frontera no-homogéneas usando la función de Green en la celda de Chang con geometría esférica 
A.3 Solución a la ecuación diferencial ordinaria de segundo orden con condiciones de frontera de tercer tipo usando la función de Green

A.4 Construcción de la función de Green para la ecuación diferencial ordinaria de segundo grado con condiciones de frontera Neumann-Dirichlet

A.5 . Prueba de simetría del problema de cerradura

A.6 Ecuaciones del ADI 2-D

A.7 Ecuaciones del ADI 3-D

A.8 Gráficos de la variable de cerradura 



\section{Antecedentes}

Se inicia este capítulo presentando algunos aspectos básicos de la dispersión, se hace una breve reseña histórica de los desarrollos realizados en el estudio del transporte dispersivo, y se presentan algunos resultados previos del coeficiente longitudinal y transversal de dispersión.

El proceso de dispersión de un soluto en un medio poroso es de vital importancia en ciertas áreas de la ingeniería. En ingeniería química, entender y modelar la dispersión permite diseñar adecuadamente un reactor de lecho empacado; en ingeniería ambiental, permite entender y predecir la contaminación de los acuíferos por solventes químicos, productos derivados del petróleo provenientes de ductos y tanques y por lixiviados de los depósitos de basura.

\subsection{Dispersión}

La dispersión en medios porosos se trata mediante técnicas de cambio de escalas. En muchos casos, estas técnicas conducen a una ecuación que gobierna el proceso de dispersión en escala macroscópica, teniendo como variables dependientes a las propiedades de la sustancia que se dispersa (difusividad, por ejemplo). En general, en estas ecuaciones aparece un tensor efectivo de segundo orden que, conforme se ve más adelante es el que caracteriza el proceso de dispersión en escala microscópica.

La dispersión puede definirse como un conjunto de procesos que contribuyen a transportar un soluto, una suspensión o un flujo de calor en el seno de un fluido en movimiento. Es posible distinguir dos tipos de dispersión con respecto a la dirección principal del flujo: la dispersión longitudinal, resultado de las heterogeneidades de las componentes de la velocidad microscópica paralelas al flujo, y la dispersión transversal que resulta de las componentes ortogonales de la velocidad microscópica a la dirección del flujo. 
La dispersión de un soluto en un medio poroso es un proceso complejo estrechamente relacionado con la interfase sólido-fluido y el régimen de flujo. En la literatura existen tres tipos de análisis para hacer frente a este el problema:

1 Acercamiento probabilístico (Greenkorn \& Kessler, 1972; Harina \& Greenkorn, 1978; Saffman, 1967; De Josselin de Jong G., 1958) en donde el medio poroso es visto como una serie de poros cuyos radios, longitudes y orientación son determinados por funciones de distribución de probabilidad. La probabilidad de que una "partícula de fluido" pase alrededor de un poro es considerada como proporcional flujo volumétrico en ese poro. Dando las funciones de distribución de probabilidad uno puede fácilmente determinar la propagación del pulso y por lo tanto las componentes del tensor de dispersión. Aunque estos modelos son útiles para determinar la sensibilidad de los coeficientes de dispersión a las propiedades físicas de cada de las fases del medio poroso, desprecian los detalles del campo de flujo y resultan poco útiles para tratar problemas de dispersión con adsorción y desorción.

2 El del método del promedio volumétrico (Whitaker, 1967), en el cual el medio poroso es modelado por un medio espacialmente periódico, conduce a ecuaciones de transporte promediadas espacialmente y además establece un método para la predicción del tensor de dispersión.

3 El acercamiento que usa modelos geométricos. El medio poroso es aproximado por una ubicuidad de tubos capilares (Whitaker, 1983). Una de las desventajas es que el modelo es restrictivo en cuanto a la geometría del medio poroso. Este acercamiento es inspirado por el estudio de un solo tubo capilar (Taylor, 1953; 1954; Aris, 1956; Sartory, 1978). Este modelo se puede extender a diversos arreglos de tubos capilares y permite explorar el efecto del tamaño del poro en los coeficientes de transporte.

Como se ha mencionado, el primer trabajo en tratar el problema de la dispersión fue el de Taylor en 1953, en el que estudió la dispersión de un soluto contenido en un fluido que 
penetra por un tubo capilar cilíndrico, donde las dimensiones de las partículas del soluto son mucho menores que las dimensiones fisicas del tubo. Se abordaron los casos de flujo laminar y turbulento, tanto teórico como experimentalmente. De la teoría resultó que la dispersión era en el sentido longitudinal del tubo (dispersión longitudinal) y proporcional a $P e^{2}$, lo que fue confirmado experimentalmente. Aunque dicho trabajo no esté directamente relacionado con la dispersión en medios porosos, puede extenderse a un medio poroso modelándolo como un conjunto de tubos capilares. En este mismo trabajo, Taylor muestra que el centro de masa de la distribución del soluto se mueve con la velocidad promedio del flujo, verifica además que la distribución es simétrica con respecto al centro de masa.

Aris en 1956, retoma el trabajo de Taylor determinando las ecuaciones de los momentos de probabilidad de la distribución del soluto. En este desarrollo, la ecuación del primer momento corresponde a la velocidad de desplazamiento del centro de masa de la distribución de soluto, mientras que la ecuación del segundo momento da la dispersión del soluto en torno al mismo.

Horn en 1971 generalizó la técnica y los conceptos desarrollados por Aris y los aplicó al estudio de la dispersión de masa en columnas cromatográficas.

Brenner en 1980, desarrolló de forma más detallada los conceptos conocidos hasta entonces y aplicó la técnica de los momentos a medios porosos espacialmente periódicos. En este trabajo, a partir de la determinación de las ecuaciones que gobiernan los principales momentos de la distribución del soluto, desarrolló una técnica que permite determinar el comportamiento asintótico de la distribución del soluto inyectado en el medio poroso. Brenner demuestra que para un medio poroso espacialmente peribdico, la velocidad de Darcy es independiente de la celda unitaria considerada y que el tensor de dispersión es un invariante macroscópico. 


\subsection{Algunos resultados teóricos y experimentales}

Los resultados de Taylor y Aris (Teoria de Taylor-Aris) predicen que en la dispersión en capilares, la variación del coeficiente de dispersión longitudinal con el número de Péclet es de la forma $D_{x x} / D_{e f f} \approx 1+\frac{1}{48} P e^{2}$. En los trabajos de Eidsath et al. (1983), se consideran medios porosos espacialmente periódicos bidimensionales, el comportamiento obtenido del coeficiente longitudinal de dispersión es del tipo $D_{x x} / D_{e f f} \approx 0.7 P e^{1.7}$, para valores de Péclet mayores que 10. En este último caso, se emplearon celdas unitarias de arreglos de cilindros dispuestos en línèa y en quintetos. Eidsath realizó el cálculo numérico de los variables de cerradura para arreglos periódicos de cilindros alineados usando un método de elemento finito para resolver los problemas de las variables de cerradura del proceso de dispersión pasiva. Las secciones transversales de los cilindros son circulares, en los resultados presentados la forma en cómo están dispuestos influencia significativamente el cálculo del tensor de dispersión. Sin embargo, los datos experimentales de Gunn y Pryce (1969) para medios porosos tridimensionales reales, presentan un comportamiento del tensor longitudinal de dispersión del tipo $D_{x x} / D_{\text {eff }} \approx 0.7 P e^{1.2}$. Con el fin de obtener resultados que concordaran con los resultados experimentales, Plumb y Whitaker (1988), realizaron un estudio donde se incluyeron las heterogeneidades del medio debido a variaciones del campo de porosidades mediante celdas con arreglos ordenados y desordenados. En este trabajo, los autores aplicaron el método del promedio volumétrico, lo que los llevó a obtener resultados más próximos a los experimentales. Launder y Massey (1978) empleando el método de diferencias finitas y Quintard y Whitaker (1994) con un método de volumen finito obtuvieron también los coeficientes de transporte asociados a la dispersión. Las simulaciones realizadas por Amaral Souto y Moyne (1996) con medios periódicos bidimensionales modelados por celdas unitarias con arreglos presentaron una dependencia del coeficiente longitudinal de dispersión en función del número de Péclet más próxima de los resultados experimentales. En la Tabla 1.1 se presentan algunos resultados encontrados en la literatura, del comportamiento del coeficiente longitudinal de dispersión en función del número de Péclet. 
Tabla 1.1 Resultados previos para el coeficiente de dispersión longitudinal

\begin{tabular}{|c|c|c|}
\hline Investigador & Características & $D_{x x} / \mathscr{D}_{e f f}$ \\
\hline $\begin{array}{c}\text { Taylor } \\
1953 \\
\end{array}$ & Flujo laminar en un tubo capilar & $1+\frac{1}{48} P e^{2}$ \\
\hline $\begin{array}{c}\text { Wooding } \\
1960\end{array}$ & $\begin{array}{l}\text { Flujo Poiseuille entre dos placas } \\
\text { horizontales }\end{array}$ & $1+\frac{1}{120} P e^{2}$ \\
\hline $\begin{array}{c}\text { Gunn \& Pryce } \\
1960\end{array}$ & $\begin{array}{l}\text { Mediciones experimentales en } \\
\text { arreglos aleatorios y regulares }\end{array}$ & $0.7 P e^{1.2}$ si $P e \geq 1$ \\
\hline $\begin{array}{c}\text { Eidsath et al. } \\
1983\end{array}$ & $\begin{array}{l}\text { En un arreglo bidimensional de } \\
\text { cilindros en línea }\end{array}$ & $0.7 P e^{1.7}$ si $P e \geq 10$ \\
\hline $\begin{array}{c}\text { Plumb \& Whitaker } \\
1988\end{array}$ & Medio poroso desordenado & $4.0 P e^{1.2}$ \\
\hline $\begin{array}{c}\text { Edwards et al. } \\
1991\end{array}$ & $\begin{array}{l}\text { Medio poroso ordenado en } \\
\text { arreglos de cilindros }\end{array}$ & $\approx P e^{1.761}$ \\
\hline $\begin{array}{c}\text { Amaral et al. } \\
1997\end{array}$ & Distribución aleatoria de cubos & $\approx P e^{1.6}$ si $P e \geq 10$ \\
\hline $\begin{array}{c}\text { Buyuktas \& Wallender } \\
2004\end{array}$ & $\begin{array}{l}\text { Flujo laminar en un arreglo } \\
\text { ordenado de discos }\end{array}$ & $0.0267 P e^{1.854}$ si $0.1 \leq P e \leq 100$ \\
\hline $\begin{array}{c}\text { Buyuktas \& Wallender } \\
2004\end{array}$ & $\begin{array}{l}\text { Flujo laminar en un arreglo } \\
\text { desordenado de discos }\end{array}$ & $0.0924 P e^{1.356}$ si $0.1 \leq P e \leq 100$ \\
\hline
\end{tabular}





\section{Problema de investigación}

En este proyecto de investigación se estudia el proceso de dispersión (difusión y convección) pasiva en un medio poroso rígido e impermeable, para una solución diluida del solvente $A$, considerada como un fluido newtoniano e incompresible. La ecuación de dispersión de medio efectivo se obtiene a partir de las ecuaciones espacio-temporales del proceso de dispersión en las fases del medio poroso, y del uso del método del promedio volumétrico (Whitaker, 1999). El método del promedio volumétrico permite la predicción de los coeficientes efectivos de dispersión, mediante la solución de un problema de cerradura, definido en una región representativa de la microestructura del medio poroso.

\subsection{Objetivos del problema}

El objetivo general de este trabajo es predecir los coeficientes efectivos de dispersión en un medio poroso, usando la celda unitaria de Chang como simplificación de la celda representativa del medio poroso homogéneo.

Los objetivos particulares son:

- Predecir en forma aproximada los coeficientes de transporte mediante expresiones analíticas (Funciones de Green).

- Predecir en forma aproximada los coeficientes de transporte mediante el uso de métodos numéricos ( $\mathrm{ADI}$ ).

- Comparar con resultados previos.

Para comprobar la validez de la predicción analítica de los coeficientes efectivos de dispersión, se emplea la celda de Chang con geometría esférica, y para las componentes del 
campo de velocidad se usan las expresiones algebraicas obtenidas bajo la condición de flujo potencial y reptante.

\subsection{Justificación del problema}

Las ecuaciones de medio efectivo junto con los coeficientes de transporte efectivos evitan el tratar directamente con las complicaciones asociadas con la microestructura de los sistemas multifásicos.

A pesar de que las soluciones analíticas basadas en la celda unitaria propuesta, no conducen al valor exacto de los coeficientes efectivos de transporte, nos dan una idea de los parámetros más importantes sobre su orden de magnitud. Además permiten un ahorro de cómputo y hacen factible la solución de problemas de interés práctico. 


\section{Ecuación y coeficientes de medio efectivo del proceso de dispersión pasiva}

En este capitulo se presentan las ecuaciones de medio efectivo del proceso de dispersión pasiva, resultantes de la aplicación del método del promedio volumétrico a las ecuaciones espacio-temporales de dicho proceso. Se presentan también las expresiones de los coeficientes efectivos de transporte en términos del vector de cerradura, que se obtiene de la solución problema de cerradura en una región representativa del medio poroso homogéneo. El análisis de este proceso de transporte está basado en el capítulo 3 del libro "El método del promedio volumétrico" (Whitaker 1999): Dispersión en medios porosos.

\subsection{Ecuaciones espacio-temporales y de medio efectivo del proceso de dispersión pasiva}

Equation Chapter 3 Section 1

Considérese el proceso de transporte de dispersión (difusión y convección) pasiva de una solución diluida del solvente $A$, con densidad constante, en un medio poroso rígido e impermeable, como el ilustrado en la Figura 3.1. La palabra pasiva indica que, en la interfase fluido-sólido no hay ni reacción química ni adsorción, y como el medio es impermeable, tampoco hay transferencia de masa de la fase fluida a la fase sólida. Bajo estas suposiciones, el transporte local del soluto $A$ en la región macroscópica (con longitud característica, $L$ ), mostrada en la Figura 3.2 es gobernado por el siguiente problema de valores a la frontera

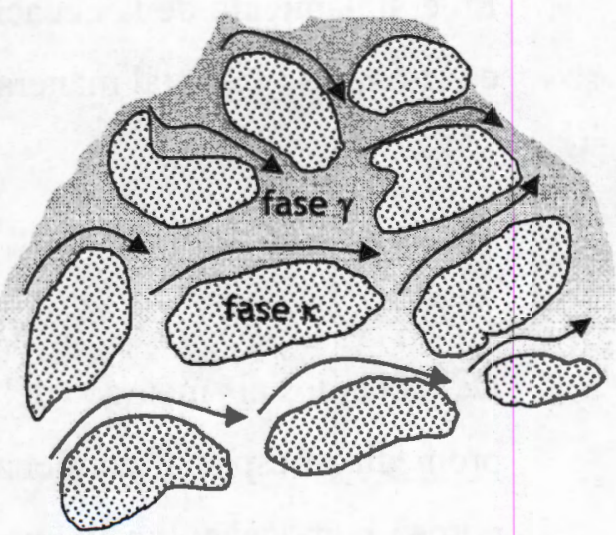

Figura 3.1 Dispersión en un medio poroso impermeable

$$
\frac{\partial c_{A \gamma}}{\partial t}+\nabla \cdot\left(\mathbf{v}_{r} c_{A \gamma}\right)=\nabla \cdot\left(\mathscr{\mathscr { P }} \nabla c_{A \gamma}\right) \text { en la fase } \gamma
$$


C. F. 1

$$
-\mathbf{n}_{r r} \cdot \mathscr{D} \nabla c_{A \gamma}=0 \text { en } \mathscr{C A}
$$

C. F. 2

$$
c_{A y}=\mathscr{F}(\mathbf{r}, t) \quad \text { en } \mathscr{d}_{\gamma e}
$$

C.I.

$$
c_{A y}=\mathscr{P}(\mathbf{r}) \quad \text { en } t=0
$$

La fase fluida corresponde a la fase $\gamma$ y al sólido rígido e impermeable se le asocia la fase $\kappa$. $\mathscr{A}_{\gamma \kappa}$ representa la superficie de la interfase $\gamma-\kappa$ contenida en la región macroscópica y $\mathscr{A}_{\gamma e}$ corresponde a la superficie de entradas y salidas de la región macroscópica. La ecuación (3.1.1) es válida para soluciones diluidas para las cuales $c_{A \gamma} \ll c_{\gamma}$. En esta misma ecuación $\mathscr{D}_{\gamma}$ es la difusividad de la mezcla de $N c$ componentes definida como sigue

$$
\frac{1}{\mathscr{D}_{Y}}=\sum_{\substack{B=1 \\ B \neq A}}^{N c} \frac{x_{B_{\gamma}}}{\mathscr{D}_{A B}}
$$

En el tratamiento de la ecuación de convección-difusión se considera un fluido newtoniano e incompresible de tal manera que la ecuación de continuidad toma la siguiente forma

$$
\nabla \cdot v_{y}=0 \text { en la fase } \gamma
$$

De acuerdo al método del promedio volumétrico (Whitaker, 1999), el proceso de promediado espacial comienza asociando a cada punto del espacio ocupado por el medio poroso homogéneo un volumen promediante $\mathscr{V}$ (ver Figura 3.2), el cual es una región del sistema macroscópico de longitud característica $L$, que es invariante con respecto al tiempo y al espacio. Su longitud característica es su radio $r_{0}$, que en la mayoría de los sistemas, debe satisfacer la restricción: $l_{\gamma} \ll r_{0} \ll L$. 


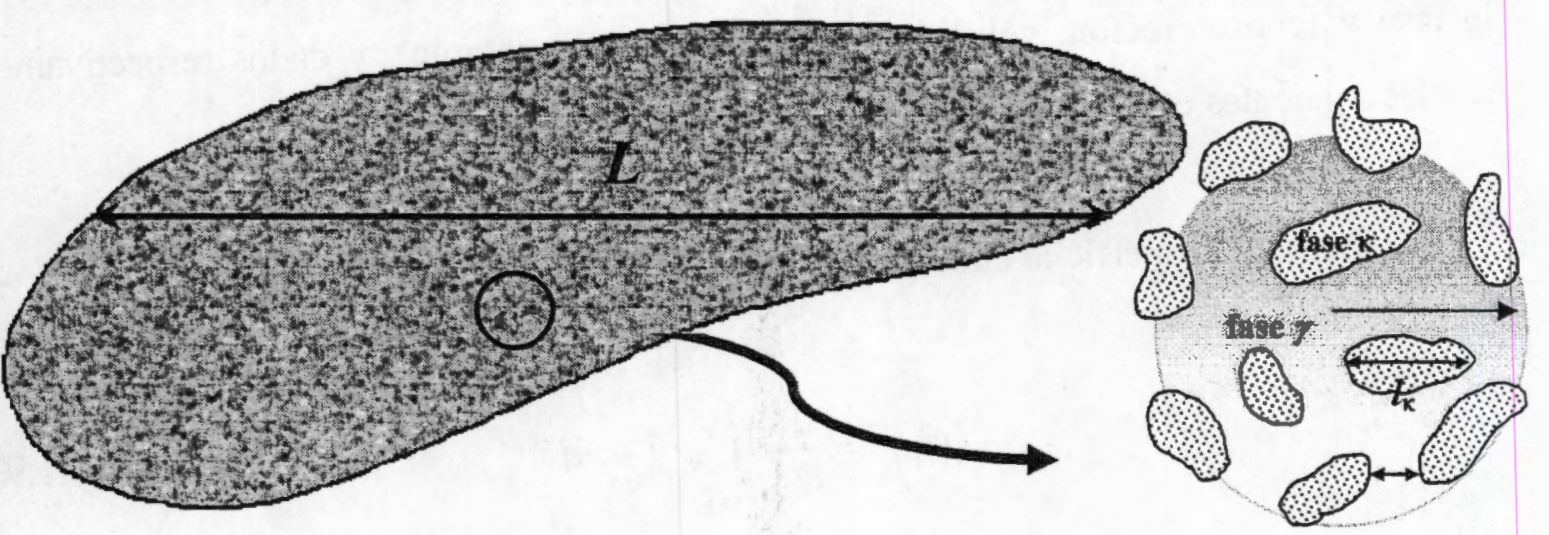

Figura 3.2 Región macroscópica y volumen promediante $\mathscr{V}$

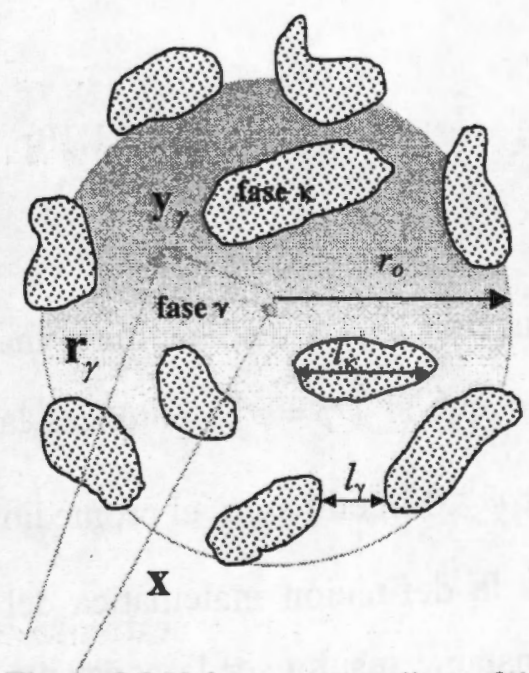

Figura 3.3 Volumen promediante of

Los detalles del volumen promediante se presentan en la Figura 3.3, en donde el vector $\mathbf{x}$ localiza el centro del volumen promediante, $\mathbf{r}_{\gamma}$ sitúa cualquier punto en la fase $\gamma$ y $\mathbf{y}_{\gamma}$, localiza los puntos en la fase $\gamma$ con respecto al centro del volumen promediante.

Para un medio poroso, el volumen promediante se puede expresar de la siguiente manera

$$
\mathscr{V}=V_{r}(\mathbf{x})+V_{k}(\mathbf{x})
$$

Donde $V_{\gamma}(\mathbf{x})$ representa el volumen de la fase $\gamma$ contenida en el volumen promediante. La fracción de volumen de la fase $\gamma$ con respecto al volumen promediante, llamada porosidad está dada por:

$$
\varepsilon_{\gamma}=\frac{V_{\gamma}(\mathbf{x})}{\mathscr{V}}
$$

El siguiente paso consiste en homogeneizar espacialmente las ecuaciones de conservación en el volumen promediante $\mathscr{T}$, aplicando las definiciones de los operadores integrales de promedio superficial e intrínseco, para alguna una propiedad $\psi_{r}$ del sistema multifásico en 
la fase $\gamma$ (concentración, velocidad, temperatura, por ejemplo), y dados respectivamente por las siguientes ecuaciones

- Promedio superficial en la fase $\gamma$

$$
\left.\left\langle\psi_{Y}\right\rangle\right|_{\mathbf{x}}=\left.\frac{1}{\mathscr{Q}{ }_{V_{Y}(\mathbf{x})}} \psi_{\gamma}\right|_{\mathbf{x}+\mathbf{y}_{Y_{Y}}} d V
$$

- Promedio intrínseco en la fase $\gamma$

$$
\left.\left\langle\psi_{r}\right\rangle^{y}\right|_{x}=\left.\frac{1}{V_{r}(\mathbf{x})} \int_{V_{r}(\mathrm{x})} \psi_{\gamma}\right|_{\mathbf{x}+\mathrm{y}_{\gamma}}
$$

Cabe mencionar, el promedio superficial, dado por la ecuación (3.1.9), no permite una buena representación de la propiedad $\psi_{\gamma}$ en la fase $\gamma$, dado que si $\psi_{\gamma}=\psi_{\gamma}^{0}$, entonces la propiedad superficial promedio no sería igual a $\psi_{y}^{0}$, sino a $\varepsilon_{\gamma} \psi_{\gamma}^{0}$. Sin embargo, el promedio intrínseco, dado por la ecuación (3.1.10), va de acuerdo a la definición matemática del operador promedio, pues el promedio intrínseco de una constante resulta ser la constante misma.

La relación entre los promedios volumétricos intrínseco y superficial está dada por:

$$
\left\langle\psi_{\gamma}\right\rangle=\varepsilon_{\gamma}\left\langle\psi_{\gamma}\right\rangle^{\gamma}
$$

Es importante mencionar que en la mayoría de los desarrollos, se desprecian las variaciones de la difusividad molecular en el volumen promediante. $\mathrm{Al}$ aplicar los operadores promedio a las ecuaciones de conservación puntuales, se obtienen promedios de gradientes, los cuales son manipulados mediante el teorema del promediado espacial (Whitaker, 1967), que puede 
ser visto como una versión tridimensional de la regla de Leibniz para intercambiar el orden de integración y diferenciación.

$$
\left\langle\nabla \psi_{\gamma}\right\rangle=\nabla\left\langle\psi_{\gamma}\right\rangle+\frac{1}{\mathscr{V}} \int_{A \Re \kappa} \mathbf{n}_{\gamma \varkappa} \psi_{\gamma} d A
$$

Al aplicar los pasos antes mencionados y el teorema del promediado espacial a las ecuaciones (3.1.1) y (3.1.6), se obtienen las siguientes expresiones

$$
\begin{aligned}
& \underbrace{\frac{\varepsilon_{\gamma} \partial\left\langle c_{A \gamma}\right\rangle^{\gamma}}{\partial t}}_{\text {caunulación }}+\underbrace{\nabla \cdot\left\langle\mathbf{v}_{\gamma} c_{A \gamma}\right\rangle}_{\text {convección }}=\underbrace{\nabla \cdot\left[\mathscr{D}_{\gamma}\left(\varepsilon_{\gamma} \nabla\left\langle c_{A \gamma}\right\rangle^{\gamma}+\left\langle c_{A \gamma}\right\rangle^{\gamma} \nabla \varepsilon_{\gamma}+\frac{1}{\mathscr{V}} \int_{A_{n}} \mathbf{n}_{\gamma \kappa} c_{A \gamma} d A\right)\right]}_{\text {difusión }} \\
& \underbrace{+\frac{1}{\mathscr{V}} \int_{A_{x}} \mathbf{n}_{m \cdot} \cdot \mathscr{P} \nabla c_{A \gamma} d A}_{\text {flux interfacial }} \\
& \nabla \cdot\left(\varepsilon_{\gamma}\left\langle\mathbf{v}_{\gamma}\right\rangle^{\gamma}\right)=0
\end{aligned}
$$

Es posible simplificar la ecuación (3.1.13), usando la condición de frontera en la interfase dada por la ecuación (3.1.2) en el término del flux interfacial. Para tratar el término de transporte convectivo y para expresar la integral de superficie en el término difusivo en función del promedio intrínseco de la concentración, empleamos la definición de la descomposición espacial de la concentración y del campo de velocidad, dadas por

$$
\begin{aligned}
& c_{A \gamma}=\left\langle c_{A \gamma}\right\rangle^{\gamma}+\tilde{c}_{A \gamma} \\
& \mathbf{v}_{\gamma}=\left\langle\mathbf{v}_{\gamma}\right\rangle^{\gamma}+\tilde{\mathbf{v}}_{\gamma}
\end{aligned}
$$


La desviación espacial representa una descomposición de escalas de longitud, es decir, los cambios en la propiedad intrínseca promedio son apreciables a escalas de la longitud característica $L$, mientras que los de la desviación espacial lo son a escalas de $l_{r}$

Sustituyendo las ecuaciones. (3.1.15)-(3.1.16) en la ecuación. (3.1.13), y usando la ecuación de continuidad promediada dada por la ecuación. (3.1.14), se obtiene la siguiente expresión

$$
\begin{aligned}
\frac{\partial\left\langle c_{A \gamma}\right\rangle^{\gamma}}{\partial t}+\left\langle\mathbf{v}_{\gamma}\right\rangle^{\gamma} \cdot \nabla\left\langle c_{A \gamma}\right\rangle^{\gamma}= & \nabla \cdot\left(\mathscr{P} \nabla\left\langle c_{A \gamma}\right\rangle^{\gamma}\right)+\varepsilon_{\gamma}^{-1} \nabla \cdot\left(\frac{\mathscr{D}}{\mathscr{V}} \int_{A_{r r}} \mathbf{n}_{\gamma r} \tilde{c}_{A \gamma} d A\right) \\
& +\varepsilon_{\gamma}^{-1} \nabla \varepsilon_{\gamma} \cdot\left(\mathscr{\mathscr { P }} \nabla\left\langle c_{A \gamma}\right\rangle^{\gamma}\right)-\varepsilon_{\gamma}^{-1} \nabla \cdot\left\langle\tilde{v}_{\gamma} \tilde{c}_{A \gamma}\right\rangle
\end{aligned}
$$

En la ecuación (3.1.17), se desprecian los términos de orden superior que contienen a $\tilde{c}_{A \gamma}, \mathrm{y}$ al realizar un análisis de órdenes de magnitud, se encuentra que esto es posible siempre que se cumpla con las siguientes restricciones

$$
l_{\gamma} \ll r_{0}, \quad r_{0}^{2} \ll L_{\delta} L_{c 1}
$$

Para cerrar la ecuación (3.1.17), es decir, para expresar $\tilde{c}_{A y}$ en términos de las variables promedio, se resta la ecuación de conservación de masa puntual, ecuación (3.1.1), a la ecuación promediada espacialmente, ecuación (3.1.17). De esta manera, se obtiene que el problema de cerradura en estado cuasi-estacionario está dado por

$$
\begin{aligned}
& \mathbf{v}_{\gamma} \cdot \nabla \tilde{c}_{A \gamma}+\tilde{\mathbf{v}}_{\gamma} \cdot \nabla\left\langle c_{A \gamma}\right\rangle^{\gamma}=\nabla \cdot\left(\mathscr{D} \nabla \tilde{c}_{A \gamma}\right) \quad \text { en la fase } \gamma \\
& -\mathbf{n}_{r x} \cdot \mathscr{D} \nabla \tilde{c}_{A \gamma}=\mathbf{n}_{r r} \cdot \mathscr{D} \gamma\left\langle\nabla c_{A \gamma}\right\rangle^{\gamma} \text { en } A_{r} \\
& \tilde{c}_{A y}=f(\mathbf{r}, t) \quad \text { en } A_{\gamma e}
\end{aligned}
$$


Al realizar un análisis de órdenes de magnitud, se encuentra que el problema de valores a la frontera dado por la ecuaciones (3.1.19)-(3.1.21), es válido siempre que se satisfagan las siguientes restricciones:

$$
\mathscr{D}_{\gamma} t^{*} \mid \delta_{y}^{2} \gg 1, l_{\gamma} \ll L
$$

Sí el medio poroso es espacialmente periódico, basta resolver el problema en una región representativa de éste, y la condición de frontera en $A_{\gamma}$ para la desviación espacial de la concentración, puede ser reemplazada por la siguiente condición de periodicidad:

$$
\tilde{c}_{A \gamma}\left(\mathbf{r}+\mathbf{l}_{i}\right)=\tilde{c}_{A \gamma}(\mathbf{r}) \quad i=1,2,3
$$

La ecuación constitutiva para $\tilde{c}_{A \gamma}$ expresada en términos de la fuente $\nabla\left\langle c_{A \gamma}\right\rangle^{\gamma}$ es

$$
\tilde{c}_{A \gamma}=\mathbf{b}_{\gamma} \cdot \nabla\left\langle c_{A \gamma}\right\rangle^{\gamma}+\pi_{\gamma}
$$

Donde $\mathbf{b}_{\boldsymbol{y}}$ es el vector de cerradura, determinado por el siguiente problema de contorno

$$
\begin{aligned}
\tilde{\mathbf{v}}_{\gamma}+\mathbf{v}_{\gamma} \cdot \nabla \mathbf{b}_{\gamma}=\mathscr{P}_{p} \nabla^{2} \mathbf{b}_{\gamma} \quad \text { en la fase } \gamma \\
-\mathbf{n}_{r x} \cdot \mathscr{P}_{\gamma} \nabla \mathbf{b}_{\gamma}=\mathbf{n}_{r r} \mathscr{P}_{\gamma} \quad \text { en } A_{r r} \\
\mathbf{b}_{\gamma}\left(\mathbf{r}+\mathbf{l}_{i}\right)=\mathbf{b}_{\gamma}(\mathbf{r}) \quad i=1,2,3
\end{aligned}
$$

Donde $\mathbf{l}_{i}$ son los vectores de lattice, $i=1,2,3$. 
La forma cerrada de la ecuación de convección-difusión está dada por

$$
\varepsilon_{\gamma} \frac{\partial\left\langle c_{A \gamma}\right\rangle^{\gamma}}{\partial t}+\left\langle\mathbf{v}_{\gamma}\right\rangle^{\gamma} \cdot \nabla\left\langle c_{A \gamma}\right\rangle^{\gamma}=D_{\gamma}^{*}: \nabla \nabla\left\langle c_{A \gamma}\right\rangle^{\gamma}
$$

El tensor de dispersión total que aparece en la ecuación (3.1.28) se define como

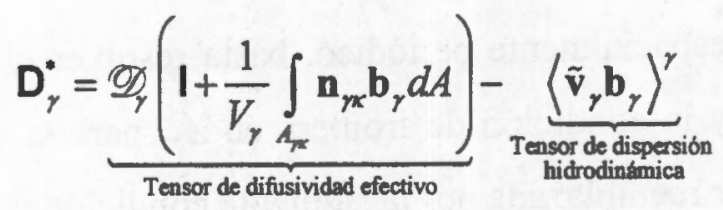

Debido a las suposiciones hechas en el la aplicación del promedio volumétrico al proceso de dispersión pasiva, el campo de velocidad corresponde a la solución de las ecuaciones de Navier-Stokes en la fase fluida de la celda unitaria representativa, para un fluido newtoniano, incompresible y con viscosidad constante.

\subsection{Adimensionalización}

\section{Equation Chapter 3 Section 2}

Existe una gran variedad de formas, para realizar la adimensionalización del problema de cerradura dado por las ecuaciones, (3.1.25)-(3.1.27), las cuales repercuten en la definición del número de Péclet. Los parámetros que permiten hacer adimensional el problema de cerradura de dispersión pasiva son parámetros característicos del medio poroso, tales como, una longitud característica de la fase fluida $\left(d_{c}\right)$ o de la fase sólida y la velocidad característica de la fase fluida, que en la mayoría de los casos corresponde a la velocidad promedio intrínseca de la fase fluida $\left(\left\langle v_{r}\right\rangle^{\gamma}\right)$. De esta manera, se definen las siguientes variables dimensionales

$$
\mathbf{y}=\frac{\mathbf{r}}{d_{c}}, \quad \mathbf{U}_{\gamma}=\frac{\mathbf{v}_{\gamma}}{\left\langle v_{\gamma}\right\rangle^{\gamma}}, \quad \tilde{\mathbf{U}}_{\gamma}=\frac{\tilde{\mathbf{v}}_{\gamma}}{\left\langle v_{\gamma}\right\rangle^{\gamma}}, \quad \mathbf{B}_{\gamma}=\frac{\mathbf{b}_{\gamma}}{d_{c}}
$$


En la literatura, los coeficientes de dispersión total en medios porosos son reportados en términos de dos definiciones del número de Péclet, éstos son:

- número de Péclet de la partícula $\left(P e_{p}\right)$ definido por

$$
P e_{p}=\frac{\left\langle v_{\gamma}\right\rangle^{\gamma} d_{p}}{\mathscr{D}}\left(\frac{\varepsilon_{\gamma}}{1-\varepsilon_{\gamma}}\right)
$$

Referido a la porosidad del medio poroso, y al diámetro efectivo $\left(d_{p}\right)$ de la partícula expresado como sigue

$$
d_{p}=6 \frac{V_{p}}{A_{p}}
$$

Donde $V_{p}$ es el volumen de la partícula y $A_{p}$ es el área de la superficie de la partícula. Para el caso de la celda de Chang con geometría esférica, el diámetro de la partícula es igual al diámetro de la esfera de la fase sólida, esto es $d_{p}=2 r_{o}$.

- número de Péclet de la celda $\left(P e_{c}\right)$, dado por

$$
P e_{c}=2 \frac{\left\langle v_{\gamma}\right\rangle^{y} l_{y}}{\mathscr{P}_{\gamma}}
$$

Referido a la longitud característica de la fase fluida.

Si se requiere el número de Péclet de la partícula, se dimensiona el problema de cerradura con

$$
d_{c}=d_{p}\left(\frac{\varepsilon_{y}}{1-\varepsilon_{\gamma}}\right)
$$


Y si el número de Péclet de la celda es requerido, la longitud característica con la que se adimensionaliza es

$$
d_{c}=l_{r}
$$

De acuerdo a la definición de las variables adimensionales, el problema de cerradura adimensionalizado está dado por el siguiente problema de valores a la frontera

$$
\begin{gathered}
P e\left(\tilde{\mathbf{U}}_{\gamma}+\mathbf{U}_{\gamma} \cdot \nabla \mathbf{B}_{\gamma}\right)=\nabla^{2} \mathbf{B}_{\gamma} \text { en } V_{\gamma} \\
-\mathbf{n}_{\gamma x} \cdot \nabla \mathbf{B}_{\gamma}=\mathbf{n}_{\gamma x} \text { en } A_{\gamma x} \\
\mathbf{B}_{\gamma}\left(\mathbf{y}+\mathbf{l}_{i}\right)=\mathbf{B}_{\gamma}(\mathbf{y}) \quad i=1,2,3
\end{gathered}
$$

Por otro lado, la ecuación de continuidad y la condición de frontera mecánica en su forma adimensional están dadas por:

$$
\begin{gathered}
\nabla \cdot \mathbf{U}(\mathbf{y})=0 \quad \mathbf{y} \in V_{\gamma} \\
\mathbf{U}(\mathbf{y})=0 \quad \mathbf{y} \in A_{\gamma / x}
\end{gathered}
$$

Basándose en la expresión del tensor de dispersión total, dado por la ecuación (3.1.29), y haciendo uso del operador de promedio intrínseco dado por la ecuación (3.1.10), se tiene que el tensor de dispersión total adimesionalizado está dado por

$$
\frac{\mathbf{D}_{\gamma}^{*}}{\mathscr{P}_{\gamma}}=\mathrm{I}+\frac{d_{c}^{3}}{V_{\gamma}} \int_{d_{r}} \mathbf{n}_{\gamma} \mathbf{B}_{\gamma} d A-P e \frac{d_{c}^{3}}{V_{\gamma}} \int_{V_{\gamma}} \tilde{\mathbf{U}}_{\gamma} \mathbf{B}_{\gamma} d V
$$


Como el objetivo es predecir los coeficientes de dispersión longitudinal y transversal, que corresponden a la entrada del tensor efectivo de dispersión total cuyas direcciones son la paralela y la ortogonal a la dirección principal de la velocidad respectivamente, entonces, no es necesario determinar todas las componentes del vector de cerradura. Basta con determinar las componentes asociadas a la dirección paralela y ortogonal al flujo. De esta manera, sea $\lambda$ (paralela u ortogonal a la dirección principal del flujo) la dirección de interés. Así, el problema de cerradura en su forma dimensional para la componente $\lambda$ del vector de cerradura está dado por:

$$
\begin{gathered}
P e\left[\tilde{U}_{\lambda}(\mathbf{y})+\mathbf{U} \cdot \nabla B_{\lambda}(\mathbf{y})\right]=\nabla^{2} B_{\lambda}(\mathbf{y}) \quad \mathbf{y} \in V_{\gamma} \\
-\mathbf{n}_{r m} \cdot \nabla B_{\lambda}(\mathbf{y})=n_{\lambda}(\mathbf{y}) \quad \mathbf{y} \in A_{r k} \\
\mathbf{B}_{\gamma}\left(\mathbf{y}+\mathbf{l}_{i}\right)=\mathbf{B}_{r}(\mathbf{y}) \quad i=1,2,3
\end{gathered}
$$

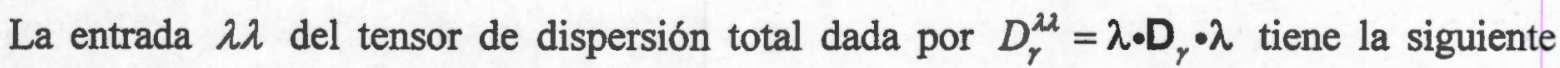
expresión

$$
\frac{D_{\gamma \lambda}^{*}}{\mathscr{D}_{\gamma}}=1+\frac{d_{c}^{3}}{V_{\gamma}} \int_{A_{r \pi}} B_{\lambda} \mathbf{n}_{\gamma \pi} \cdot \lambda d A-P e \frac{d_{c}^{3}}{V_{\gamma}} \int_{V_{\gamma}} \tilde{U}_{\lambda} B_{\lambda} d V
$$

donde $\lambda$ es el vector unitario asociado a la dirección de interés del flujo, $\lambda=\mathbf{i}, \mathbf{j}, \mathbf{k}$ 



\section{Solución analítica al problema de cerradura en la celda unitaria de Chang}

La solución a una ecuación diferencial parcial lineal, es diferente de la función cero, debido a los términos no homogéneos y a las condiciones de frontera no homogéneas, a las cuales está sujeta. La solución para cada uno de estos problemas de contorno, puede expresarse en términos de una función llamada función de Green, que describe la influencia de los términos no homogéneos de la ecuación diferencial parcial, y de las condiciones de frontera no homogéneas.

En este capítulo se presenta por un lado, la definición de la celda de Chang, y el desarrollo para obtener la solución al problema de cerradura del proceso de dispersión pasiva en términos de la función de Green, para la celda unitaria de Chang de geometría arbitraria, empleando esencialmente el teorema de la divergencia del cálculo vectorial, y se exponen paralelamente las propiedades de la función de Green. Por otro lado, se demuestra que el problema de cerradura del proceso de dispersión pasiva de un fluido newtoniano e incompresible es equivalente a un problema de valores a la frontera, descrito por la ecuación de Poisson, ambos definidos en el mismo dominio. Se deduce la expresión de las componentes del tensor de dispersión total en términos de la solución obtenida del problema de cerradura. Y finalmente, se encuentra la solución del problema de cerradura en términos de la función de Green, en la celda de Chang con geometría esférica, mediante la equivalencia de este problema con la ecuación de Poisson, usando expansiones en series de Fourier en los esféricos armónicos.

\subsection{La celda de Chang y el problema de cerradura} Equation Chapter 4 Section 1

Un medio poroso homogéneo puede ser modelado a través de una celda unitaria representativa, la cual puede simplificarse por una celda compuesta por dos regiones concéntricas $\left(\Omega_{1} \subset \Omega_{2}\right)$, descritas por una geometría análoga (dos cilindros concéntricos, 
dos esferas concéntricas, por ejemplo), ver Figura 4.1. La región contenida $\Omega_{1}$ representa la fase sólida, y la región $\Omega_{2}-\Omega_{1}$, corresponde a la fase fluida; esta celda es conocida como la celda de Chang. Dicha simplificación, permite reemplazar la condición de periodicidad en la frontera externa de la celda, por una condición Dirichlet. En un principio, esta idea fue utilizada por Chang (1982) y ha sido utilizada con éxito por Ochoa et al. $(1991,1994)$ en la predicción de coeficientes efectivos de transporte.

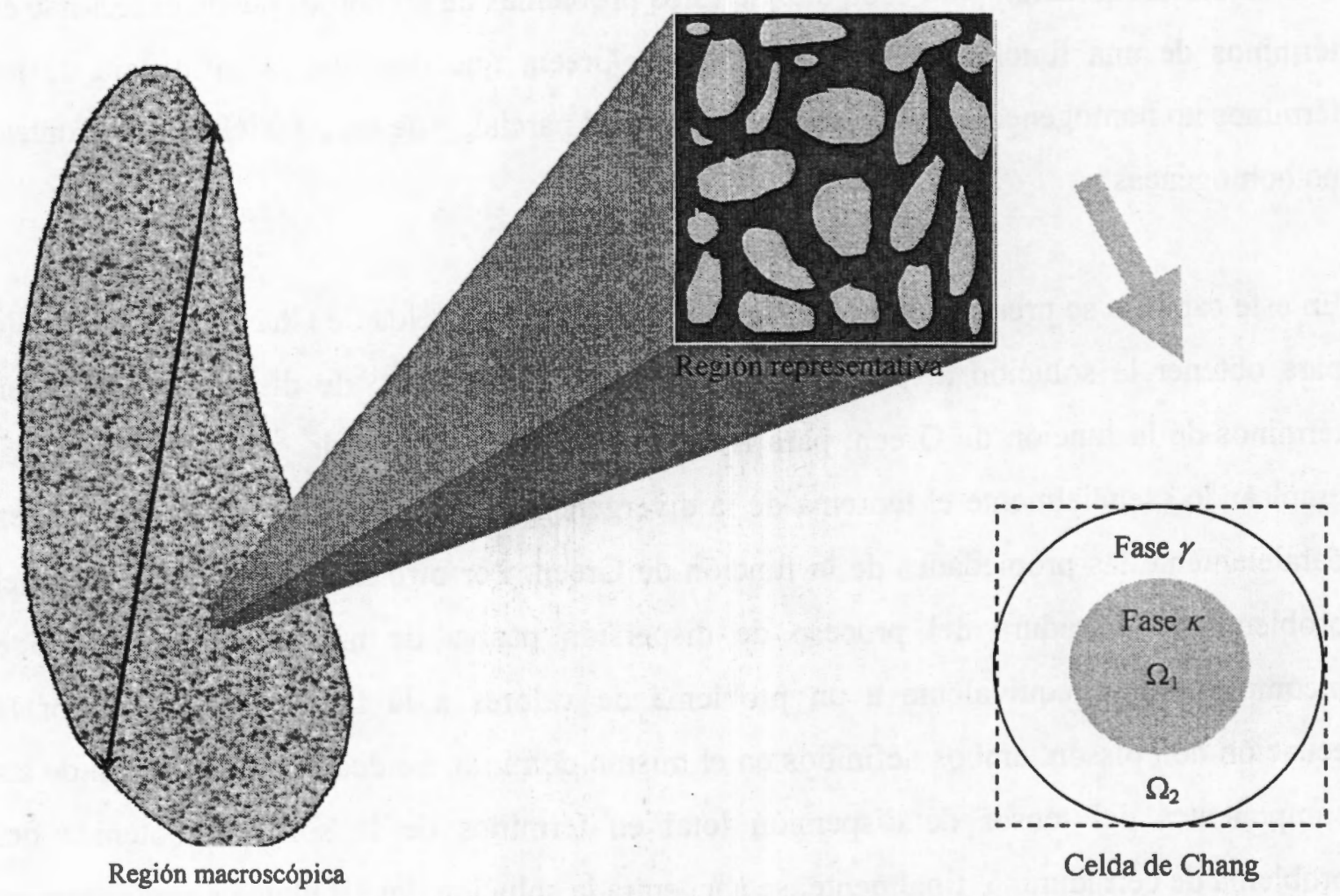

Figura 4.1: Celda de Chang como simplificación de la región representativa del medio poroso

En la Figura 4.1 se muestra una representación de la celda de Chang, si se toma esta celda como simplificación de la región representativa del medio poroso, entonces la condición de periodicidad del problema de cerradura, dada por la ecuación (3.2.9), se reemplaza por una condición de Dirichlet en la frontera externa $A_{r e}$ de la celda de Chang. De esta forma, el problema de cerradura adimensionalizado en la celda de Chang es

$$
\begin{aligned}
\operatorname{Pe}\left(\tilde{\mathbf{U}}_{\gamma}+\mathbf{U}_{\gamma} \cdot \nabla \mathbf{B}_{\gamma}\right) & =\nabla^{2} \mathbf{B}_{\gamma} \text { en } V_{\gamma} \\
-\mathbf{n}_{\gamma \pi} \cdot \nabla \mathbf{B}_{\gamma} & =\mathbf{n}_{\gamma \kappa} \text { en } \dot{A}_{\gamma \kappa}
\end{aligned}
$$




$$
\mathbf{B}_{r}(\mathbf{y})=0 \quad \text { en } A_{r e}
$$

\subsection{Solución al problema de cerradura usando la función de Green} Equation Chapter 4 Section 2

Como se menciona en el capítulo anterior, interesa resolver el problema de cerradura para la componente $\lambda$ del vector de cerradura $\left(B_{\lambda}: V_{y} \rightarrow \mathbb{R}\right)$, usando las funciones de Green, dado por el siguiente problema de valores a la frontera

$$
\operatorname{Pe}\left(\tilde{U}_{\lambda}+\mathrm{U} \cdot \dot{\nabla} B_{\lambda}\right)=\nabla^{2} B_{\lambda} \quad \text { en } V_{\gamma}
$$

Considerando que el problema de cerradura tiene por dominio la celda unitaria de Chang, definida por la región geométrica $V_{\gamma}$ que, corresponde a la fase fluida, cuya frontera está conformada por la interfase $A_{p}$ y por la frontera externa denominada $A_{r e}$. Entonces, las condiciones de frontera son

$$
\begin{gathered}
-\mathbf{n}_{r x} \cdot \nabla B_{\lambda}(\mathbf{y})=n_{\lambda} \quad \mathbf{y} \in A_{r k} \\
B_{\lambda}(\mathbf{y})=0 \quad \mathbf{y} \in A_{r e}
\end{gathered}
$$

Se define la función de Green como la solución de

$$
-P e \mathbf{U} \cdot \nabla G=\nabla^{2} G+\delta\left(\mathbf{y}-\mathbf{y}_{0}\right) \quad \text { en } V_{r}
$$

Las condiciones de frontera de este problema corresponden a las condiciones de frontera homogéneas inducidas por el problema de cerradura, por lo que éstas están dadas por

$$
\begin{gathered}
-\mathbf{n}_{y x} \cdot \nabla G\left(\mathbf{y}, \mathbf{y}_{0}\right)=0 \quad \mathbf{y} \in A_{y k} \\
G\left(\mathbf{y}, \mathbf{y}_{0}\right)=0 \quad \mathbf{y} \in A_{r e}
\end{gathered}
$$


El operador de dispersión y el operador adjunto de dispersión se definen como

$$
\begin{gathered}
L^{*}=P e \mathrm{U} \cdot \nabla-\nabla^{2} \\
L^{* *}=-P e \mathrm{U} \cdot \nabla-\nabla^{2}
\end{gathered}
$$

Considerando las funciones $A: V_{y} \rightarrow \mathbb{R}$ y $B: V_{y} \rightarrow \mathbb{R}$, y aplicando la definición de los operadores de dispersión y adjunto de dispersión, se tiene la siguiente expresión

$$
A L^{* *}(B)-B L^{*}(A)=-P e \mathbf{U} \cdot \nabla(A B)+B \nabla^{2} A-A \nabla^{2} B
$$

Integrando la ecuación (4.2.9) en el dominio $V_{\gamma}$ y de las fórmulas de Green se tiene

$$
\iiint_{V_{r}}\left[A L^{* *}(B)-B L^{*}(A)\right] d V=-P e \oiint_{\partial V_{r}} A B \mathbf{U} \cdot \mathbf{n} d S+P e \iiint_{V_{r}} A B \nabla \cdot \mathbf{U} d V+\oiint_{\partial V_{r}}(B \nabla A-A \nabla B) \cdot \mathbf{n} d S
$$

Por otro lado, de la ecuación de continuidad para un fluido incompresible, se sabe que el campo de velocidad satisface que

$$
\nabla \cdot \mathrm{U}=0 \text { en } V_{r}
$$

De esta manera, la fórmula de Green para la ecuación de dispersión se reduce a

$$
\iiint_{V_{r}}\left[A L^{* *}(B)-B L^{*}(A)\right] d V=\oiint_{\partial V_{r}}[(B \nabla A-A \nabla B)-P e A B \mathbf{U}] \cdot \mathbf{n} d S
$$

A continuación, se demostrará la reciprocidad de la función de Green en $V_{y}$, es decir,

$$
G\left(\mathbf{y}_{0}, \mathbf{y}_{1}\right)=G\left(\mathbf{y}_{1}, \mathbf{y}_{0}\right)
$$

Para la demostración de dicha propiedad de la función de Green, en la fórmula de Green para la ecuación de dispersión se sustituyen las expresiones siguientes 


$$
\begin{aligned}
& A=G\left(\mathbf{y}, \mathbf{y}_{0}\right) \\
& B=G\left(\mathbf{y}, \mathbf{y}_{1}\right)
\end{aligned}
$$

Los problemas de valores a la frontera para $A$ y $B$ son

$$
\begin{aligned}
& L^{*}(A)=\delta\left(\mathbf{y}-\mathbf{y}_{0}\right) \\
& L^{* *}(B)=\delta\left(\mathbf{y}-\mathbf{y}_{1}\right)
\end{aligned}
$$

De esta manera, sustituyendo las ecuaciones (4.2.14)-(4.2.17) en la fórmula de Green para de dispersión (4.2.12), y sabiendo que los problemas de valores a la frontera de $A$ y $B$ tienen condiciones de frontera homogéneas, se tiene por un lado que

$$
\iiint_{V_{r}}\left[G\left(\mathbf{y}, \mathbf{y}_{0}\right) \delta\left(\mathbf{y}-\mathbf{y}_{1}\right)-G\left(\mathbf{y}, \mathbf{y}_{1}\right) \delta\left(\mathbf{y}-\mathbf{y}_{0}\right)\right] d V=0
$$

La propiedad de filtración de la función delta de Dirac para $f: \mathbb{R}^{3} \rightarrow \mathbb{R}$ está dada por

$$
\iiint_{\mathbb{R}^{3}} f\left(\mathbf{y}_{0}\right) \delta\left(\mathbf{y}-\mathbf{y}_{0}\right) d V=f(\mathbf{y})
$$

Para poder hacer uso de la propiedad anterior, se define la extensión de la función de Green $\Gamma$ en todo $\mathbb{R}^{3}$ como sigue

$$
\Gamma\left(\mathbf{x}, \mathbf{x}_{0}\right)=\left\{\begin{array}{cc}
0 & \mathbf{y} \notin V_{r} \\
G\left(\mathbf{y}, \mathbf{y}_{0}\right) & \mathbf{y} \in V_{r}
\end{array}\right.
$$

De esta manera, se tiene por un lado que 


$$
\begin{aligned}
\iiint_{\mathbf{R}^{3}}\left[\Gamma\left(\mathbf{y}, \mathbf{y}_{0}\right) \delta\left(\mathbf{y}-\mathbf{y}_{1}\right)-\Gamma\left(\mathbf{y}, \mathbf{y}_{1}\right) \delta\left(\mathbf{y}-\mathbf{y}_{0}\right)\right] d V= \\
\qquad \iiint_{V_{r}}\left[G\left(\mathbf{y}, \mathbf{y}_{0}\right) \delta\left(\mathbf{y}-\mathbf{y}_{1}\right)-G\left(\mathbf{y}, \mathbf{y}_{1}\right) \delta\left(\mathbf{y}-\mathbf{y}_{0}\right)\right] d V
\end{aligned}
$$

Paralelamente, de la propiedad de filtración de la función delta de Dirac, se tiene que

$$
\iiint_{\mathbf{R}^{3}}\left[\Gamma\left(\mathbf{y}, \mathbf{y}_{0}\right) \delta\left(\mathbf{y}-\mathbf{y}_{1}\right)-\Gamma\left(\mathbf{y}, \mathbf{y}_{1}\right) \delta\left(\mathbf{y}-\mathbf{y}_{0}\right)\right] d V=\Gamma\left(\mathbf{y}_{1}, \mathbf{y}_{0}\right)-\Gamma\left(\mathbf{y}_{0}, \mathbf{y}_{1}\right) \quad \text { en } \mathbb{R}^{3}
$$

Finalmente, se obtiene que

$$
\iiint_{V_{\gamma}}\left[G\left(\mathbf{y}, \mathbf{y}_{0}\right) \delta\left(\mathbf{y}-\mathbf{y}_{1}\right)-G\left(\mathbf{y}, \mathbf{y}_{1}\right) \delta\left(\mathbf{y}-\mathbf{y}_{0}\right)\right] d V=G\left(\mathbf{y}_{1}, \mathbf{y}_{0}\right)-G\left(\mathbf{y}_{0}, \mathbf{y}_{1}\right)
$$

Al igualar las ecuaciones (4.2.18) y (4.2.23) queda demostrada la reciprocidad de la función de Green en $V_{r}$.

Para encontrar la solución al problema de dispersión usando las funciones de Green, se sustituye en la fórmula de Green de dispersión (4.2.12) las siguientes ecuaciones

\begin{tabular}{|c|c|}
\hline Problema no-homogéneo & Función de Green \\
\hline$A(\mathbf{y})=B_{\lambda}(\mathbf{y})$ & $B=G\left(\mathbf{y}, \mathbf{y}_{0}\right)$ \\
$L^{*}\left(B_{\lambda}\right)=-P e \tilde{U}_{\lambda} \quad(4.2 .24)$ & $L^{* *}(G)=\delta\left(\mathbf{y}-\mathbf{y}_{0}\right)$ \\
\hline
\end{tabular}

Se obtiene

$$
\iiint_{V_{y}}\left[B_{\lambda} \delta\left(\mathbf{y}-\mathbf{y}_{0}\right)+P e \tilde{U}_{\lambda}(\mathbf{y}) G\left(\mathbf{y}, \mathbf{y}_{0}\right)\right] d V=\oiint_{\partial V_{y}}\left[\begin{array}{r}
G\left(\mathbf{y}, \mathbf{y}_{0}\right) \nabla B_{\lambda}-B_{\lambda} \nabla G\left(\mathbf{y}, \mathbf{y}_{0}\right) \\
-P e B_{\lambda} G\left(\mathbf{y}, \mathbf{y}_{0}\right) \mathbf{U}(\mathbf{y})
\end{array}\right] \cdot \mathbf{n} d S
$$





$$
\begin{gathered}
B_{\lambda}(\mathbf{y})=-P e \iiint_{V_{y}} \tilde{U}_{\lambda}\left(\mathbf{y}_{0}\right) G\left(\mathbf{y}, \mathbf{y}_{0}\right) d V_{0} \\
+\iint_{\lambda^{\prime}}\left\{G\left(\mathbf{y}, \mathbf{y}_{0}\right) \nabla_{0} B_{\lambda}\left(\mathbf{y}_{0}\right)-B_{\lambda}\left(\mathbf{y}_{0}\right)\left[\nabla_{0} G\left(\mathbf{y}, \mathbf{y}_{0}\right)+P e G\left(\mathbf{y}, \mathbf{y}_{0}\right) \mathbf{U}\left(\mathbf{y}_{0}\right)\right]\right\} \cdot \mathbf{n} d S_{0}(4.2 .30) \\
+\iint_{A_{\lambda}}\left\{G\left(\mathbf{y}, \mathbf{y}_{0}\right) \nabla_{0} B_{\lambda}\left(\mathbf{y}_{0}\right)-B_{\bar{\lambda}}\left(\mathbf{y}_{0}\right)\left[\nabla_{0} G\left(\mathbf{y}, \mathbf{y}_{0}\right)+P e G\left(\mathbf{y}, \mathbf{y}_{0}\right) \mathbf{U}\left(\mathbf{y}_{0}\right)\right]\right\} \cdot \mathbf{n} d S_{0}
\end{gathered}
$$

Se aclara que en la interfase, el vector $\mathbf{n}_{m}$ es el vector normal a la interfase dirigido de la fase fluida $\gamma$ a la fase sólida $\kappa$, y por lo tanto, $\mathbf{n}_{m}=-\mathbf{n}$ en la interfase.

De las condiciones de frontera del problema de cerradura (4.1.2)-(4.1.3) y del problema que define a la función de Green (4.2.5)-(4.2.6), la solución del problema de cerradura dada por la ecuación (4.2.30) se simplifica a

$$
B_{\lambda}(\mathbf{y})=\underbrace{\iiint_{\lambda_{n}} G\left(\mathbf{y}, \mathbf{y}_{0}\right) n_{\lambda 0} d S_{0}}_{\text {término difusivo }}-\underbrace{P e \iiint_{V_{r}} \tilde{U}_{\lambda}\left(\mathbf{y}_{0}\right) G\left(\mathbf{y}, \mathbf{y}_{0}\right) d V_{0}}_{\text {término convectivo }}
$$

Se observa que la componente $\lambda$ del vector de cerradura presenta la contribución de la difusión y de la convección.

\subsection{Equivalencia del problema de cerradura con la ecuación de Poisson en la celda de Chang}

Equation Chapter 4 Section 3

Para demostrar que el problema de cerradura, dado por las ecuaciones (4.2.1)-(4.1.3) es equivalente a un problema de valores a la frontera definido por la ecuación de Poisson, se considera la función $\beta_{\lambda}: V_{\gamma} \rightarrow \mathbb{R}$ que corresponde a la componente $\lambda$ de un vector de cerradura $\beta_{\gamma}$ diferente de $\mathbf{B}_{\gamma}$, que también satisface el problema de cerradura y cuyo problema de contorno es el siguiente 
De las propiedades de la función delta de Dirac, la ecuación (4.2.26) puede escribirse como sigue

$$
\begin{array}{r}
B_{\lambda}\left(\mathbf{y}_{0}\right)=-P e \iiint_{V_{\gamma}} \tilde{U}_{\lambda} G\left(\mathbf{y}, \mathbf{y}_{0}\right) d V-P e \oiint_{\partial V_{y}} B_{\lambda} G\left(\mathbf{y}, \mathbf{y}_{0}\right) \mathrm{U} \cdot \mathbf{n} d S+ \\
\oiint_{\partial V_{y}}\left[G\left(\mathbf{y}, \mathbf{y}_{0}\right) \nabla B_{\lambda}-B_{\lambda} \nabla G\left(\mathbf{y}, \mathbf{y}_{0}\right)\right] \cdot \mathbf{n} d S
\end{array}
$$

Intercambiando $\mathbf{y}_{0}$ por $\mathbf{y}$ y empleando la propiedad de reciprocidad de la función de Green (4.2.13), se encuentra que

$$
\begin{array}{r}
B_{\lambda}(\mathbf{y})=-\underbrace{P e \iiint_{V_{z}} \tilde{U}_{\lambda}\left(\mathbf{y}_{0}\right) G\left(\mathbf{y}, \mathbf{y}_{0}\right) d V_{0}}_{\text {contribución del témino no-bonogéneo de la EDP }} \\
\oiint_{\partial V_{y}}\left\{G\left(\mathbf{y}, \mathbf{y}_{0}\right) \nabla_{0} B_{\lambda}\left(\mathbf{y}_{0}\right)-B_{\lambda}\left(\mathbf{y}_{0}\right)\left[\nabla_{0} G\left(\mathbf{y}, \mathbf{y}_{0}\right)+P e G\left(\mathbf{y}, \mathbf{y}_{0}\right) \mathbf{U}\left(\mathbf{y}_{0}\right)\right]\right\} \cdot \mathbf{P} d S_{0}
\end{array}
$$

contribución de les condiciones de frontere de la EDP

De la ecuación (4.2.28) se puede observar que, la función de Green, permite reflejar la influencia del término no-homogéneo y de las condiciones de frontera no-homogéneas en la solución del problema de cerradura.

Además, dado que la fase sólida en la celda de Chang es rígida e impermeable y considerando que no hay penetración en la interfase, el campo de velocidad en la interfase satisface que

$$
\mathbf{U}(\mathbf{y})=0 \quad \mathbf{y} \in A_{p \mathbf{x}}
$$

Considerando la propiedad de no penetración del fluido en la interfase (4.2.29), la solución al problema de cerradura para el proceso de dispersión pasiva en términos de la función de Green en la celda unitaria de Chang es 


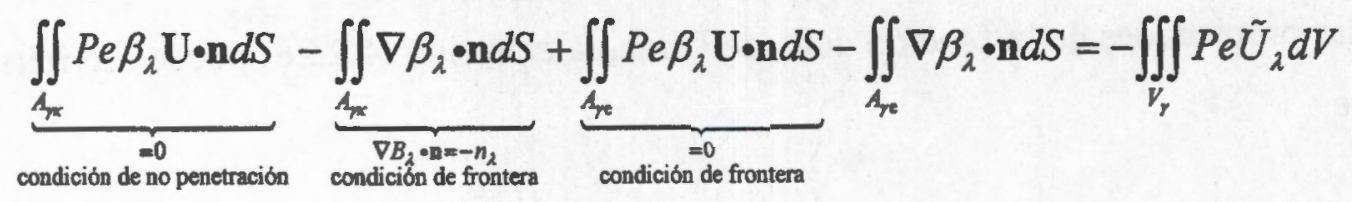

De la condición de no penetración (4.2.29), de las condiciones de frontera en la interfase $A_{p k}$ y en la frontera externa $A_{y e}$ dadas por las ecuaciones (4.3.2) y (4.3.3) respectivamente, se encuentra que el problema de cerradura de la componente $\lambda$ del vector de cerradura escrito en forma variacional es

$$
\iiint_{\lambda_{n}} \nabla \beta_{\lambda} \cdot \mathbf{n} d S+\iint_{A_{r}} \nabla \beta_{\lambda} \cdot \mathbf{n} d S=\iiint_{V_{r}} P e \tilde{U}_{\lambda} d V
$$

Aplicando el teorema de la divergencia al primer miembro de la ecuación (4.3.9), se tiene que

$$
\iiint_{V_{y}} \nabla^{2} \beta_{\lambda} d V=\iiint_{V_{y}} P e \tilde{U}_{\lambda} d V
$$

Esto demuestra que

$$
\iiint_{V_{r}} P e \nabla \cdot \beta_{\lambda} \mathbf{U} d V=\iiint_{V_{r}} P e \mathbf{U} \cdot \nabla \beta_{\lambda} d V=0
$$

Las ecuaciones (4.3.10) y (4.3.11) demuestran que el término $P e \mathrm{U} \cdot \nabla \beta_{\lambda}$ de la ecuación diferencial parcial del problema de cerradura dado por las ecuaciones (4.3.1)-(4.3.3) no tiene influencia en la solución de dicho problema: Este hecho nos lleva a pensar que la solución del problema de cerradura es la misma que la solución al siguiente problema de valores a la frontera 


$$
\operatorname{Pe}\left(\tilde{U}_{\lambda}+\mathbf{U} \cdot \nabla \beta_{\lambda}\right)=\nabla^{2} \beta_{\lambda} \quad \text { en } V,
$$

Condiciones de frontera

$$
\begin{gathered}
-\mathbf{n}_{m} \cdot \nabla \beta_{\lambda}(\mathbf{y})=n_{\lambda} \quad \mathbf{y} \in A_{\mu^{*}} \\
\beta_{\lambda}(\mathbf{y})=0 \quad \mathbf{y} \in A_{r e}
\end{gathered}
$$

De las propiedades del operador gradiente se obtiene que

$$
\nabla \cdot\left(P e \mathbf{U} \beta_{\lambda}-\nabla \beta_{\lambda}\right)=P e \mathbf{U} \cdot \nabla \beta_{\lambda}+P e \beta_{\lambda}(\nabla \cdot \mathbf{U})-\nabla^{2} \beta_{\lambda}
$$

Y de la ecuación de continuidad (4.2.11), la identidad (4.3.4) se reduce a

$$
\nabla \cdot\left(P e \mathrm{U} \beta_{\lambda}-\nabla \beta_{\lambda}\right)=P e \mathrm{U} \cdot \nabla \beta_{\lambda}-\nabla^{2} \beta_{\lambda} \quad \text { en } V_{\gamma}
$$

De esta manera, la ecuación diferencial parcial que define el problema de cerradura está dada también por

$$
\nabla \cdot\left(P e \beta_{\lambda} \mathbf{U}-\nabla \beta_{\lambda}\right)=-P e \tilde{U}_{\lambda} \quad \text { en } V_{\gamma}
$$

Integrando la ecuación (4.3.6) en el dominio $V_{r}$, se obtiene la siguiente expresión

$$
\iiint_{V_{y}} \nabla \cdot\left(P e \beta_{\lambda} \mathbf{U}-\nabla \beta_{\lambda}\right) d V=-\iiint_{V_{\gamma}} P e \tilde{U}_{\lambda} d V
$$

Empleando el teorema de la divergencia al segundo miembro de la ecuación (4.3.7) y considerando las fronteras del dominio del problema de cerradura, se encuentra 
delta de Dirac y las condiciones de frontera homogéneas asociadas para la función de Green dadas por las ecuaciones (4.3.16)-(4.3.17), se encuentra que

$$
G^{*}\left(\mathbf{y}_{1}, \mathbf{y}_{0}\right)=G^{*}\left(\mathbf{y}_{0}, \mathbf{y}_{1}\right)
$$

Para expresar la solución a la ecuación de Poisson en términos de la función de Green, se sustituye en la fórmula de Green del operador de Laplace (4.3.18), las funciones siguientes

\begin{tabular}{|c|c|}
\hline Problema no-homogéneo & Función de Green \\
\hline$A(\mathbf{y})=\beta_{\lambda}(\mathbf{y})$ & $B=G^{*}\left(\mathbf{y}, \mathbf{y}_{0}\right)$ \\
$L_{p}\left(\beta_{\lambda}\right)=-P e \tilde{U}_{\lambda} \quad(4.3 .20)$ & $L_{p}\left(G^{*}\right)=\delta\left(\mathbf{y}-\mathbf{y}_{0}\right)$ \\
\hline
\end{tabular}

Usando la propiedad de filtración de la función delta de Dirac (4.2.19), intercambiando $\mathbf{y}_{0}$ por $\mathbf{y}, \mathrm{y}$ empleando la propiedad de simetría de la función de Green, se obtiene

$$
\beta_{\lambda}(\mathbf{y})=P e \iint_{V_{y}} G^{*}\left(\mathbf{y}, \mathbf{y}_{0}\right) \tilde{U}_{\lambda}\left(\mathbf{y}_{0}\right) d V_{0}+\iint_{\partial V_{y}}\left[\beta_{\lambda}\left(\mathbf{y}_{0}\right) \nabla_{0} G^{*}\left(\mathbf{y}, \mathbf{y}_{0}\right)-G^{*}\left(\mathbf{y}, \mathbf{y}_{0}\right) \nabla_{0} \beta_{\lambda}\left(\mathbf{y}_{0}\right)\right] \cdot \mathbf{n} d S_{0}
$$

Como la frontera de la celda de Chang está compuesta por la interfase $A_{p k}$ y por la frontera externa $A_{r e}$, entonces

$$
\begin{aligned}
& \beta_{\lambda}(\mathbf{y})=\operatorname{Pe} \iiint_{V_{r}} \tilde{U}_{\lambda}\left(\mathbf{y}_{0}\right) G^{*}\left(\mathbf{y}, \mathbf{y}_{0}\right) d V_{0}
\end{aligned}
$$

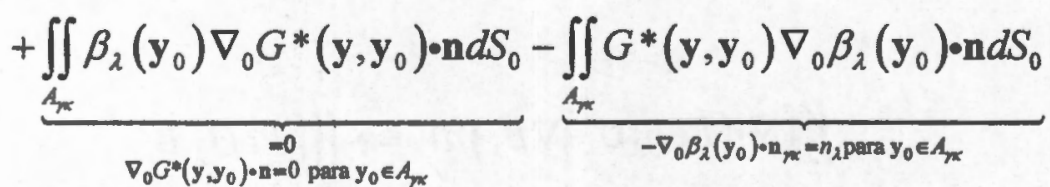

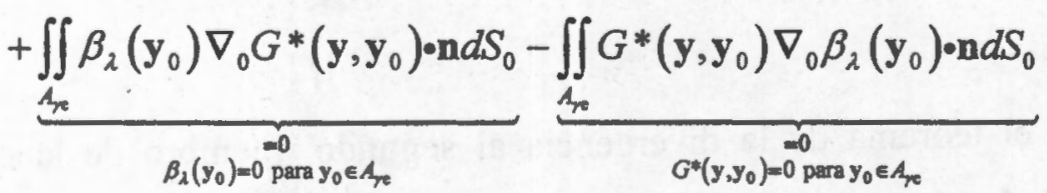




$$
\begin{gathered}
\nabla^{2} \beta_{\lambda}(\mathbf{y})=P e \tilde{U}_{\lambda}(\mathbf{y}) \quad \text { en } V_{r} \\
-\mathbf{n}_{r \cdot} \cdot \nabla \beta_{\lambda}(\mathbf{y})=n_{\lambda} \quad \mathbf{y} \in A_{r} \\
\beta_{\lambda}(\mathbf{y})=0 \quad \mathbf{y} \in A_{r e}
\end{gathered}
$$

A continuación se presenta el desarrollo para obtener la solución a la ecuación de Poisson en términos de la función de Green $G^{*}\left(\mathbf{y}, \mathbf{y}_{0}\right)$, que es solución de la ecuación

$$
\nabla^{2} G^{*}\left(\mathbf{y}, \mathbf{y}_{0}\right)=\delta\left(\mathbf{y}-\mathbf{y}_{0}\right) \quad V_{\gamma}
$$

Sujeta a las condiciones de frontera homogéneas asociadas al problema de Poisson, dadas por las ecuaciones (4.3.13)-(4.3.14),

$$
\begin{gathered}
-\mathbf{n}_{r *} \cdot \nabla G^{*}\left(\mathbf{y}, \mathbf{y}_{0}\right)=0 \quad \mathbf{y} \in A_{r k} \\
G^{*}\left(\mathbf{y}, \mathbf{y}_{0}\right)=0 \quad \mathbf{y} \in A_{r e}
\end{gathered}
$$

Como el operador de Laplace $L_{p} \equiv \nabla^{2}$, es un operador lineal adjunto, y del teorema de la divergencia se obtiene la fórmula de Green para este operador, para las funciones $A: V_{\gamma} \rightarrow \mathbb{R}$ y $B: V_{\gamma} \rightarrow \mathbb{R}$,

$$
\iiint_{V_{r}}\left[A L_{p}(B)-B L_{p}(A)\right] d V=\oiint_{\partial V_{p}}(A \nabla B-B \nabla A) \cdot \mathbf{n} d S
$$

Para demostrar la propiedad de reciprocidad de la función de Green ,se procede de manera análoga a la sección anterior, esto es, en la fórmula de Green para el operador de Laplace, dado por la ecuación (4.3.18), se hace $L_{p}(A)=L_{p}\left[G^{*}\left(\mathbf{y}, \mathbf{y}_{0}\right)\right]=\delta\left(\mathbf{y}-\mathbf{y}_{0}\right)$ y $L_{p}(B)=L_{p}\left[G^{*}\left(\mathbf{y}, \mathbf{y}_{1}\right)\right]=\delta\left(\mathbf{y}-\mathbf{y}_{1}\right)$, aplicando la propiedad de filtración de la función 
Expresando la solución en términos de los operadores del método del promedio volumétrico y de la función de Green, se encuentra la siguiente expresión

$$
B_{\lambda}(\mathbf{y})=A_{r k}\left\langle G\left(\mathbf{y}, \mathbf{y}_{0}\right) n_{\lambda 0}\right\rangle_{p r}^{0}-P e V_{r}(\mathbf{y})\left\langle\tilde{U}_{\lambda}\left(\mathbf{y}_{0}\right) G\left(\mathbf{y}, \mathbf{y}_{0}\right)\right\rangle_{0}^{\gamma}
$$

\subsection{Unicidad de los problemas equivalentes en la celda de Chang} Equation Chapter 4 Section 4

La demostración de la unicidad de la solución de los problemas de cerradura y de la ecuación se basa en el método de la energía.

\subsubsection{Problema de cerradura}

Para poder demostrar la unicidad del problema de cerradura, se cuenta con las siguientes igualdades resultantes de las propiedades de los operadores diferenciales

$$
\begin{gathered}
\nabla B_{\lambda} \cdot B_{\lambda} \mathbf{U}=\frac{1}{2} \nabla \cdot B_{\lambda}{ }^{2} \mathbf{U}-\frac{1}{2} B_{\lambda}{ }^{2} \nabla \cdot \mathbf{U} \\
B_{\lambda} \nabla \cdot\left(P e \mathrm{U} B_{\lambda}-\nabla B_{\lambda}\right)=\nabla \cdot B_{\lambda}\left(P e \mathrm{U} B_{\lambda}-\nabla B_{\lambda}\right)-\nabla B_{\lambda} \cdot P e \mathbf{U} B_{\lambda}+\left|\nabla B_{\lambda}\right|^{2}
\end{gathered}
$$

Sustituyendo la ecuación (4.4.1) en la identidad (4.4.2), se obtiene que

$$
B_{\lambda} \nabla \cdot\left(P e \mathbf{U} B_{\lambda}-\nabla B_{\lambda}\right)=\nabla \cdot B_{\lambda}\left(P e \mathbf{U} B_{\lambda}-\nabla B_{\lambda}\right)-\frac{1}{2} \nabla \cdot P e B_{\lambda}{ }^{2} \mathbf{U}+\frac{1}{2} B_{\lambda}{ }^{2} \nabla \cdot P e \mathbf{U}+\left|\nabla B_{\lambda}\right|^{2}
$$

Al integrar la ecuación (4.4.2) en el dominio $V_{\gamma}$, considerando que el número de Péclet es constante en el volumen promediante, y aplicando la ecuación de continuidad (4.2.11), el teorema de la divergencia y la propiedad de no penetración en la interfase (4.2.29), se obtiene la siguiente expresión 
De las condiciones de frontera para las funciones involucradas, se tiene que

$$
\beta_{\lambda}(\mathbf{y})=P e \iiint_{V_{z}} \tilde{U}_{\lambda}\left(\mathbf{y}_{0}\right) G^{*}\left(\mathbf{y}, \mathbf{y}_{0}\right) d V_{0}-\iint_{\lambda_{k}} G^{*}\left(\mathbf{y}, \mathbf{y}_{0}\right) n_{\lambda 0} d S_{0}
$$

Rearreglando la ecuación (4.3.24), y recordando la expresión de la solución al problema de cerradura para $B_{\lambda}$ en términos de la función de Green $G$, dada por la ecuación (4.2.31) y comparando ambos resultados, se puede concluir que los problemas son equivalentes siempre que

$$
\begin{gathered}
\beta_{\lambda}(\mathbf{y})=-B_{\lambda}(\mathbf{y}) \\
G^{*}\left(\mathbf{y}, \mathbf{y}_{0}\right)=G\left(\mathbf{y}, \mathbf{y}_{0}\right)
\end{gathered}
$$

Se ha entonces demostrado la equivalencia entre los siguientes problemas de valores a la frontera

\begin{tabular}{ll|c}
\multicolumn{2}{c|}{ Ecuación de Poisson } & \multicolumn{2}{|c}{ Problema de cerradura } \\
\hline$\nabla^{2} B_{\lambda}(\mathbf{y})=-P e \tilde{U}_{\lambda}(\mathbf{y})$ en $V_{r}$ & $(4.3 .26)$ & $P e U \cdot \nabla B_{\lambda}-\nabla^{2} B_{\lambda}=-P e \tilde{U}_{\lambda}$ en $V_{r}$ \\
$\mathbf{n}_{r m} \cdot \nabla B_{\lambda}(\mathbf{y})=n_{\lambda}, \mathbf{y} \in A_{r k}$ & $(4.3 .27)$ & $-\mathbf{n}_{r k} \cdot \nabla B_{\lambda}(\mathbf{y})=n_{\lambda}, \mathbf{y} \in A_{r k}$ \\
$B_{\lambda}(\mathbf{y})=0, \mathbf{y} \in A_{r e}$ & $(4.3 .28)$ & $B_{\lambda}(\mathbf{y})=0, \mathbf{y} \in A_{r e}$
\end{tabular}

La solución usando la fórmula de Green a estos problemas de contorno equivalentes es

$$
B_{\lambda}(\mathbf{y})=\iint_{\lambda_{x}} G\left(\mathbf{y}, \mathbf{y}_{0}\right) n_{\lambda 0} d S_{0}-P e \iiint_{V_{y}} \tilde{U}_{\lambda}\left(\mathbf{y}_{0}\right) G\left(\mathbf{y}, \mathbf{y}_{0}\right) d V_{0}
$$


(4.4.7), esta constante debe ser cero, $\eta=B_{\lambda 1}-B_{\lambda 2} \equiv 0$, lo que implica que, $B_{\lambda 1}=B_{\lambda 2}$. Esto demuestra la unicidad de la solución del problema de cerradura.

\subsubsection{Ecuación de Poisson}

De las propiedades de los operadores diferenciales se tiene la siguiente expresión

$$
A \nabla^{2} A=\nabla \cdot(A \nabla A)-|\nabla A|^{2}
$$

Integrando la ecuación (4.4.9) para $A$ en el dominio $V_{\gamma}$ con frontera $\partial V_{\gamma}$, y aplicando el teorema de la divergencia, se obtiene el teorema de Green

$$
\iiint_{V_{y}} A \nabla^{2} A d V=\iint_{\partial V_{y}} A \nabla A \cdot \mathbf{n} d S-\iiint_{V_{y}}|\nabla A|^{2} d V
$$

Procediendo de manera análoga, a la demostración de la unicidad de la solución del problema de cerradura, es decir, se supone que tanto $B_{\lambda 1}$ como $B_{\lambda 2}$ son soluciones a la ecuación de Poisson, de esta manera la diferencia $\eta=B_{\lambda 1}-B_{\lambda 2}$ cumple que la ecuación de Laplace

$$
\nabla^{2} \eta=0 \quad \text { en } V_{r}
$$

Con las siguientes condiciones de frontera

$$
\begin{gathered}
\mathbf{n}_{r m} \cdot \nabla \eta(\mathbf{y})=0, \mathbf{y} \in A_{r m} \\
\eta(\mathbf{y})=0, \mathbf{y} \in A_{r e}
\end{gathered}
$$




$$
\begin{aligned}
& \iiint_{V_{r}}\left[B_{\lambda} \nabla \cdot\left(P e \mathbf{U}-\nabla B_{\lambda}\right)\right] d V=\underbrace{\frac{P e}{2} \iint_{\lambda_{n}}\left(B_{\lambda}{ }^{2} \mathbf{U} \cdot \mathbf{n}\right) d S}_{\text {Por la condicion de no pentracion }}-\iint_{\lambda_{r}}\left(B_{\lambda} \nabla B_{\lambda} \cdot \mathbf{n}\right) d S \\
& +\frac{P e}{2} \iiint_{\lambda_{r}}\left(B_{\lambda}{ }^{2} \mathbf{U} \cdot \mathbf{n}\right) d S-\iint_{A_{r}}\left(B_{\lambda} \nabla B_{\lambda} \cdot \mathbf{n}\right) d S+\underbrace{\frac{P e}{2} \iiint_{V_{r}}\left(B_{\lambda}{ }^{2} \nabla \cdot \mathbf{U}\right) d V}_{\text {Por la ecuacion de continuided }}+\iiint_{V_{r}}\left|\nabla B_{\lambda}\right|^{2} d V
\end{aligned}
$$

Para demostrar que el problema de cerradura tiene a lo sumo una solución, se supone que las funciones $B_{\lambda 1}$ y $B_{\lambda 2}$ son ambas soluciones a dicho problema, y definiendo la diferencia de estas funciones como $\eta=B_{\lambda 1}-B_{\lambda 2}$. Entonces, la función $\eta$ queda definida por el siguiente problema de valores a la frontera

$$
\begin{gathered}
P e \mathbf{U} \cdot \nabla \eta-\nabla^{2} \eta=\nabla \cdot(P e \mathbf{U} \eta-\nabla \eta)=0 \quad \text { en } V_{\gamma} \\
\mathbf{n}_{\gamma} \cdot \nabla \eta(\mathbf{y})=0, \mathbf{y} \in A_{r x} \\
\eta(\mathbf{y})=0, \mathbf{y} \in A_{r e}
\end{gathered}
$$

Reemplazando las condiciones del problema de contorno de la función $\eta$ dadas por las ecuaciones (4.4.5)-(4.4.7) en la ecuación (4.4.4), resulta que

$$
\iiint_{V_{y}}|\nabla \eta|^{2} d V=0
$$

Como el integrando en la ecuación (4.4.8) es no-negativo y continuo (dada la continuidad de las funciones $B_{\lambda 1}$ y $B_{\lambda 2}$ ), entonces se concluye que debe ser idénticamente nulo. Por lo tanto, $\eta$ debe ser constante, y de la condición de frontera de $\eta$ en $A_{\gamma e}$ dada por la ecuación 
Sustituyendo la ecuación (4.5.1) en la expresión de la entrada $\lambda \lambda$ del tensor adimensional de dispersión total, dada por la ecuación (3.2.16), y agrupando términos, se obtiene que

$$
\frac{D_{r \lambda 2}^{*}}{\mathscr{D}_{\gamma}}=A_{2} P e^{2}+A_{1} P e+A_{0}+1
$$

En donde

$$
\begin{aligned}
& A_{2}=-\frac{d_{c}^{3}}{V_{y}} \int_{V_{r}} \tilde{U}_{\gamma \lambda} B_{\lambda}^{\text {convectivo }} d V \\
& A_{1}=\frac{d_{c}^{3}}{V_{r}}\left[\int_{A_{m}} B_{\lambda}^{\text {convectivo }} \mathbf{n}_{r} \cdot \lambda d A-\int_{V_{r}} \tilde{U}_{p \lambda} B_{\lambda}^{d j \text { jusivo }} d V\right] \\
& A_{0}=-\frac{d_{c}^{3}}{V_{\gamma}} \int_{A_{m}} B_{\lambda}^{\text {dijusivo }} \mathbf{n}_{y x} \cdot \lambda d A
\end{aligned}
$$

De la ecuación (4.5.4), se observa que la componente $\lambda \lambda$ del coeficiente adimensional de dispersión total corresponde a un polinomio de grado 2 en el número de Péclet, y que los coeficientes, dados por la ecuación (4.5.5), contienen la información de la microestructura del medio poroso y del campo de velocidad en la fase fluida.

\subsection{Función de Green y coeficiente de dispersión total en la celda de Chang con geometría esférica}

Equation Chapter 4 Section 6

\subsubsection{Celda de Chang con geometría esférica}

El volumen total de la celda de Chang (ver Figura 4.2) con geometría esférica es

$$
\mathscr{V}=\frac{4}{3} \pi \xi_{e}^{3}
$$

El volumen de la fase sólida en la celda de Chang está dado por 
Dada la estructura del problema de valores a la frontera para $\eta$, la ecuación (4.4.10) para esta misma función se reduce a

$$
\iiint_{V_{y}}|\nabla \eta|^{2} d V=0
$$

De manera análoga a la sección 4.3.1, el integrando debe ser ịéntico a la función cero, lo que implica que, $\eta$ debe ser cero, dada la condición de frontera de $\eta$ en $A_{\text {re }}$, $\eta=B_{\lambda 1}-B_{\lambda 2} \equiv 0$, lo que demuestra la unicidad de la solución de la ecuación de Poisson.

Finalmente, se puede concluir que, como los problemas de cerraduras y de la ecuación de Poisson presentan unicidad en su solución, y sabiendo que estos problemas son equivalentes, entonces, la solución de un problema es la única solución del otro y viceversa.

\subsection{Coeficientes de dispersión total}

\section{Equation Chapter 4 Section 5}

De acuerdo a la expresión de la componente $\lambda$ del vector de cerradura en términos de la función de Green, es posible descomponer la ecuación (4.3.32) en dos términos, uno correspondiente a la difusión y el otro a la dispersión hidrodinámica. De esta manera, se tiene la siguiente ecuación

$$
B_{\lambda}(\mathbf{y})=B_{\lambda}^{\text {dijusino }}(\mathbf{y})+P e B_{\lambda}^{\text {convectivo }}(\mathbf{y})
$$

En donde

$$
\begin{gathered}
B_{\lambda}^{\text {dijusivo }}(\mathbf{y})=\iint_{\lambda_{r k}} G\left(\mathbf{y}, \mathbf{y}_{0}\right) n_{\lambda 0} d S_{0} \\
B_{\lambda}^{\text {convectivo }}(\mathbf{y})=-\iiint_{V_{r}} \tilde{U}_{\lambda}\left(\mathbf{y}_{0}\right) G\left(\mathbf{y}, \mathbf{y}_{0}\right) d V_{0}
\end{gathered}
$$


Los vectores canónicos del sistema de coordenadas esférico $\mathbf{e}_{\xi}$ y $\mathbf{e}_{\theta}$ en términos de los vectores canónicos del sistema de coordenado cartesiano están dados por

$$
\begin{aligned}
& \mathbf{e}_{\xi}=\operatorname{sen} \theta \cos \phi \mathbf{i}+\operatorname{sen} \theta \operatorname{sen} \phi \mathbf{j}+\cos \theta \mathbf{k} \\
& \mathbf{e}_{\theta}=\cos \theta \cos \phi \mathbf{i}+\cos \theta \operatorname{sen} \phi \mathbf{j}-\operatorname{sen} \theta \mathbf{k}
\end{aligned}
$$

Para la adimensionalización, si se requiere el número de Péclet de la partícula, se adimensionaliza el problema de cerradura con

$$
d_{c}=2 r_{o}\left(\frac{\varepsilon_{\gamma}}{1-\varepsilon_{\gamma}}\right)
$$

Y cuando se necesite el número de Péclet de la celda, la longitud característico con la que se adimensionaliza es

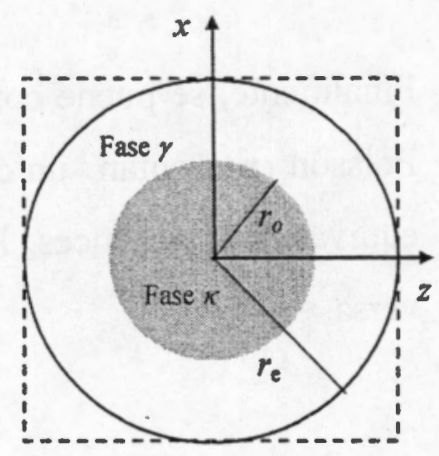

Figura 4.3: Celda esférica de Chang

$$
d_{c}=2 r_{e}
$$

\subsubsection{Solución a la ecuación de Poisson con condiciones de frontera no- homogéneas usando la función de Green en la celda de Chang con geometría esférica}

Equation Chapter 4 Section 6

El problema de contorno descrito por la ecuación de Poisson (4.3.26)-(4.3.28) en coordenadas esféricas está dado por 


$$
V_{k}=\frac{4}{3} \pi \xi_{p}^{3}
$$

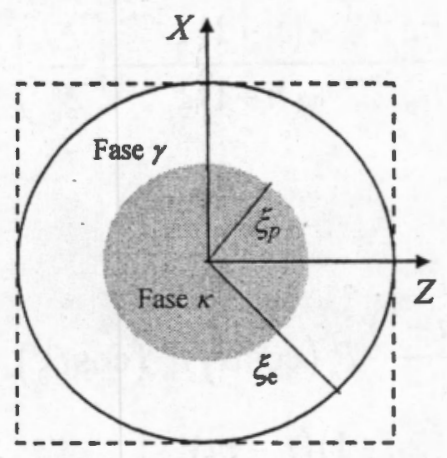

Figura 4.2: Celda esférica de Chang adimensional

El volumen de la fase fluida en la celda de Chang con geometría esférica es

$$
V_{\gamma}=\frac{4}{3} \pi\left(\xi_{e}^{3}-\xi_{p}^{3}\right)
$$

De esta manera, la porosidad (fracción volumen de la fase $\gamma$ ) tiene la expresión siguiente

$$
\varepsilon_{r}=1-\left(\frac{\xi_{p}}{\xi_{e}}\right)^{3}
$$

De la ecuación (4.6.4), el radio externo se puede expresar en función de la porosidad de acuerdo a la siguiente ecuación

$$
\xi_{e}=\xi_{p} \sqrt[3]{\frac{1}{1-\varepsilon_{y}}}
$$

El vector normal a la interfase $\gamma \kappa$ es el vector canónico radial del sistema de coordenadas esféricas en dirección contraria, esto es

$$
\mathrm{n}_{\pi}=-\mathbf{e}_{\xi}
$$




$$
g_{n}\left(\xi, \xi_{0}\right)=\left\{\begin{array}{l}
\frac{1}{(2 n+1) \xi_{e}} \frac{n \xi_{p}^{2 n+1}+(n+1) \xi_{0}^{2 n+1}}{n \xi_{p}^{2 n+1}+(n+1) \xi_{e}^{2 n+1}}\left(\frac{\xi_{0}}{\xi_{e}}\right)^{n+1}\left[\left(\frac{\xi}{\xi_{e}}\right)^{n}-\left(\frac{\xi}{\xi_{e}}\right)^{-n-1}\right] \xi_{0} \leq \xi \\
\frac{1}{(2 n+1) \xi_{e}} \frac{n \xi_{p}^{2 n+1}+(n+1) \xi_{p}^{2 n+1}}{n \xi_{p}^{2 n+1}+(n+1) \xi_{e}^{2 n+1}}\left(\frac{\xi}{\xi_{e}}\right)^{n+1}\left[\left(\frac{\xi_{0}}{\xi_{e}}\right)^{n}-\left(\frac{\xi_{0}}{\xi_{e}}\right)^{-n-1}\right] \xi \leq \xi_{0}
\end{array}\right.
$$

$$
\begin{aligned}
K_{n}\left(\theta, \phi ; \theta_{0}, \phi_{0}\right)= & \frac{(2 n+1)}{4 \pi} P_{n}(\cos \theta) P_{n}\left(\cos \theta_{0}\right) \\
& +\sum_{m=1}^{n} \frac{(2 n+1)(n-m) !}{2 \pi(n+m) !} P_{n}^{m}(\cos \theta) P_{n}^{m}\left(\cos \theta_{0}\right) \cos m\left(\phi-\phi_{0}\right)
\end{aligned}
$$

El kernel cumple también la siguiente igualdad

$$
K_{n}\left(\theta, \phi ; \theta_{0}, \phi_{0}\right)=\frac{2 n+1}{4 \pi} P_{n}\left(\cos \theta_{0}^{\prime}\right)
$$

Donde $\theta_{0}^{\prime}$ es el ángulo formado por las direcciones $(\theta, \phi)$ y $\left(\theta_{0}, \phi_{0}\right)$ y está definido por

$$
\cos \theta_{0}^{\prime}=\cos \theta \cos \theta_{0}+\operatorname{sen} \theta \operatorname{sen} \theta_{0} \cos \left(\phi-\phi_{0}\right)
$$

La función de Green en términos del ángulo $\theta_{0}^{\prime}$ puede expresarse como sigue

$$
G\left(\xi, \theta, \phi ; \xi_{0}, \theta_{0}, \phi_{0}\right)=\frac{1}{4 \pi} \sum_{n=0}^{\infty}(2 n+1) g_{n}\left(\xi, \xi_{0}\right) P_{n}\left(\cos \theta_{0}^{\prime}\right)
$$

\section{Independencia axial simétrica de la solución del problema de cerradura}

En el apéndice A.2 se demuestra que, sí el término no homogéneo del problema de cerradura es axialmente simétrico, al igual que la condición de frontera en $\xi=\xi_{p}$, es decir, 


$$
\begin{array}{r}
\frac{1}{\xi^{2}} \frac{\partial}{\partial \xi}\left(\xi^{2} \frac{\partial B_{\lambda}}{\partial \xi}\right)+\frac{1}{\xi^{2} \operatorname{sen} \theta} \frac{\partial}{\partial \theta}\left(\operatorname{sen} \theta \frac{\partial B_{\lambda}}{\partial \theta}\right)+\frac{1}{\xi^{2} \operatorname{sen}^{2} \theta} \frac{\partial^{2} B_{\lambda}}{\partial \phi^{2}}=-P e \tilde{U}_{\lambda}(\xi, \theta, \phi) \\
(\xi, \theta, \phi) \in\left(\xi_{p}, \xi_{e}\right) \times(0, \pi) \times(0,2 \pi)
\end{array}
$$

El problema de contorno está sujeto a las condiciones de frontera de tipo Neumann en la interfase y Dirichlet en el radio externo, éstas se escriben como sigue

$$
\begin{gathered}
\frac{\partial B_{\lambda}}{\partial \xi}\left(\xi_{p}, \theta, \phi\right)=f(\theta, \phi) \\
B_{\lambda}\left(\xi_{e}, \theta, \phi\right)=0
\end{gathered}
$$

La solución al problema de valores a la frontera, dado por las ecuaciones (4.6.1)-(4.6.3), en términos de la función de Green es

$$
\begin{array}{r}
B_{\lambda}(\xi, \theta, \phi)=P e \int_{0}^{2 \pi} \int_{0}^{\pi} \int_{\xi_{p}}^{\pi} \tilde{U}_{\lambda}\left(\xi_{0}, \theta_{0}, \phi_{0}\right) G\left(\xi, \theta, \phi ; \xi_{0}, \theta_{0}, \phi_{0}\right) \xi_{0}^{2} \operatorname{sen} \theta_{0} d \xi_{0} d \theta_{0} d \phi_{0} \\
+\int_{0}^{2 \pi} \int_{0}^{\pi} f(\theta, \phi) G\left(\xi, \theta, \phi ; \xi_{p}, \theta_{0}, \phi_{0}\right) \operatorname{sen} \theta d \theta d \phi
\end{array}
$$

Los detalles de cómo se obtiene la función de Green a partir del problema de valores a la frontera descrito por las ecuaciones (4.6.1)-(4.6.3) puede consultarse en el apéndice A.2 de esté trabajo. De donde resulta que la función de Green está definida por la siguiente expresión

$$
G\left(\xi, \theta, \phi ; \xi_{0}, \theta_{0}, \phi_{0}\right)=\sum_{n=0}^{\infty} g_{n}\left(\xi, \xi_{0}\right) K_{n}\left(\theta, \phi ; \theta_{0}, \phi_{0}\right)
$$

Donde 


$$
\begin{gathered}
A_{2}=-\frac{1}{V_{\gamma}} \int_{0}^{2 \pi} \int_{0}^{\pi} \int_{\xi_{p}}^{\xi_{p}} \tilde{U}_{\gamma \lambda} B_{\lambda}^{\text {convectivo }}(\xi, \theta, \phi) \xi^{2} \operatorname{sen} \theta d \xi d \theta d \phi \\
A_{1}=-\frac{1}{V_{\gamma}}\left[\int_{0}^{2 \pi} \int_{0}^{\pi} \int_{\xi_{p}}^{\xi_{\xi}} \tilde{U}_{\gamma \lambda} B_{\lambda}^{\text {difusivo }}(\xi, \theta, \phi) \xi^{2} \operatorname{sen} \theta d \xi d \theta d \phi+\int_{0}^{2 \pi} \int_{0}^{\pi} \lambda \cdot \mathrm{e}_{\xi} B_{\lambda}^{\text {convectivo }}\left(\xi_{p}, \theta, \phi\right) \operatorname{sen} \theta d \theta d \phi\right] \\
A_{0}=-\frac{1}{V_{\gamma}} \int_{0}^{2 \pi} \int_{0}^{\pi} \lambda \cdot e_{\xi} B_{\lambda}^{d i f u s i v o}\left(\xi_{p}, \theta, \phi\right) \operatorname{sen} \theta d \theta d \phi
\end{gathered}
$$

En la Tabla 4.1 se presentan las expresiones de las componentes de la diagonal del tensor total de dispersión, junto con las condiciones de frontera en la interfase, a la cual está sujeta la ecuación de Poisson y las componentes en coordenadas cartesianas de la desviación de la velocidad espacial 
que es independiente de la variable $\phi$, entonces la solución al problema de cerradura también es axialmente simétrica.

De esta manera, la solución al problema de cerradura dada por la ecuación (4.6.4) está dada por:

$$
\begin{aligned}
B_{\lambda}(\xi, \theta)=P e \int_{0}^{\pi} \int_{\xi_{p}}^{\xi_{p}} \tilde{U}_{\lambda}\left(\xi_{0}, \theta_{0}\right) G\left(\xi, \theta, \phi ; \xi_{0}, \theta_{0}, \phi_{0}\right) \xi_{0}^{2} \operatorname{sen} \theta_{0} d \xi_{0} d \theta_{0} \\
+\int_{0}^{\pi} f\left(\xi_{p}, \theta_{0}\right) G\left(\xi, \theta ; \xi_{p}, \theta_{0}\right) \operatorname{sen} \theta_{0} d \theta_{0}
\end{aligned}
$$

En este caso se puede definir la función de Green como

$$
G\left(\xi, \theta, \phi ; \xi_{0}, \theta_{0}, \phi_{0}\right)=\frac{1}{2} \sum_{n=0}^{\infty}(2 n+1) g_{n}\left(\xi, \xi_{0}\right) P_{n}(\cos \theta) P_{n}\left(\cos \theta_{0}\right)
$$

\subsection{Coeficiente de dispersión total en la celda esférica de Chang} Equation Chapter 4 Section 7

De acuerdo a la ecuación (4.5.4) que corresponde a la expresión del coeficiente de dispersión total, la entrada $\lambda \lambda$ del tensor dispersión total efectivo está dada por

$$
\frac{D_{\gamma \lambda \lambda}^{*}}{\mathscr{D}_{r}}=A_{2} P e^{2}+A_{1} P e+A_{0}+1
$$

donde 

Tabla 4.1 Componentes $x x, y y$ y $z z$ del tensor de dispersión total

\begin{tabular}{|c|c|c|c|}
\hline & Componente $X$ & Componente $Y$ & Componente $\mathrm{Z}$ \\
\hline $\begin{array}{l}\text { Condición de } \\
\text { frontera en la } \\
\text { interfase }\end{array}$ & $\frac{\partial B_{y X}}{\partial \xi}=\operatorname{sen} \theta \cos \phi$ & $\frac{\partial B_{r^{y}}}{\partial \xi}=\operatorname{sen} \theta \operatorname{sen} \phi$ & $\frac{\partial B_{\gamma}}{\partial \xi}=\cos \theta$ \\
\hline $\begin{array}{l}\text { Desviación } \\
\text { espacial de la } \\
\text { velocidad }\end{array}$ & $\tilde{U}_{\gamma X}=\left(\tilde{U}_{\gamma^{\xi}} \operatorname{sen} \theta-\tilde{U}_{\gamma \theta} \cos \theta\right) \operatorname{sen} \phi$ & $\tilde{U}_{r Y}=\left(\tilde{U}_{\gamma^{\xi}} \operatorname{sen} \theta-\tilde{U}_{\gamma \theta} \cos \theta\right) \cos \phi$ & $\tilde{U}_{r z}=\tilde{U}_{r \xi} \cos \theta-\tilde{U}_{r \theta} \operatorname{sen} \theta$ \\
\hline $\begin{array}{l}\text { Vector de } \\
\text { cerradura }\end{array}$ & $\begin{array}{r}B_{X}(\mathbf{x})=P e \int_{0}^{2 \pi} \int_{0}^{\pi} \int_{\xi_{p}}^{\xi_{\xi}} \tilde{U}_{X}\left(\mathbf{x}_{0}\right) G\left(\mathbf{x}, \mathbf{x}_{0}\right) d V_{0} \\
+G_{1}\left(\xi, \xi_{p}\right) \operatorname{sen} \theta \cos \phi\end{array}$ & $\begin{array}{r}B_{Y}(\mathbf{x})=P e \int_{0}^{2 \pi} \int_{0}^{\pi} \int_{\xi_{p}}^{\xi_{Y}} \tilde{U}_{Y}\left(\mathbf{x}_{0}\right) G\left(\mathbf{x}, \mathbf{x}_{0}\right) d V_{0} \\
+G_{1}\left(\xi, \xi_{p}\right) \operatorname{sen} \theta \cos \phi\end{array}$ & $\begin{array}{r}B_{Z}(\mathbf{x})=P e \int_{0}^{2 \pi} \int_{0}^{\pi} \int_{\xi_{p}}^{\xi} \tilde{U}_{z}\left(\mathbf{x}_{0}\right) G\left(\mathbf{x}, \mathbf{x}_{0}\right) d V_{0} \\
+G_{1}\left(\xi, \xi_{p}\right) \cos \theta\end{array}$ \\
\hline $\begin{array}{l}\text { Coeficiente de } \\
\text { difusividad } \\
\text { efectiva }\end{array}$ & $\frac{D_{e f f}^{2 \pi}}{\mathscr{D}_{\gamma}}=1-\frac{\xi^{2}}{V_{y}} \int_{0}^{2 \pi} \int_{0}^{\pi} B_{y X} \operatorname{sen}^{2} \theta \cos \phi d \theta d \phi$ & $\frac{D_{e f f}^{y y}}{\mathscr{D}_{\gamma}}=1-\frac{\xi^{2}}{V_{\gamma}} \int_{0}^{2 \pi} \int_{0}^{\pi} B_{r Y} \operatorname{sen}^{2} \theta \operatorname{sen} \phi d \theta d \phi$ & $\frac{D_{e f f}^{2 z}}{\mathscr{D Q}_{\gamma}}=1-\frac{2 \pi \xi^{2}}{V_{\gamma}} \int_{0}^{\pi} B_{y z} \cos \theta \operatorname{sen} \theta d \theta$ \\
\hline $\begin{array}{c}\text { Coeficiente } \\
\text { hidrodinámico } \\
\text { de dispersión }\end{array}$ & $\frac{D_{\gamma}^{\alpha x}}{\mathscr{D}_{\gamma}}=-\frac{P e}{V_{\gamma}} \int_{0}^{2 \pi} \int_{0}^{\pi} \int_{0}^{\xi_{q}} \tilde{U}_{\gamma X} B_{\gamma X} \xi^{2} \operatorname{sen} \theta d \xi d \theta d \phi$ & $\frac{D_{r}^{y y}}{\mathscr{D}_{\gamma}}=-\frac{P e^{2}}{V_{\gamma}} \int_{0}^{2 \pi} \int_{0}^{\pi} \int_{\xi_{p}}^{\xi_{\xi}} \tilde{U}_{r Y} B_{r r} \xi^{2} \operatorname{sen} \theta d \xi d \theta d \phi$ & $\frac{D_{r}^{z z}}{\mathscr{D}_{\gamma}}=-2 \pi \frac{P e}{V_{r}} \int_{0}^{\pi} \int_{\xi_{p}}^{\xi_{q}} \tilde{U}_{r z} B_{r z} \xi^{2} \operatorname{sen} \theta d \xi d \theta$ \\
\hline
\end{tabular}

Donde $G\left(\mathbf{x}, \mathbf{x}_{0}\right)=\sum_{n=0}^{\infty} G_{n}\left(\xi ; \xi_{0}\right) K_{n}\left(\theta, \phi ; \theta_{0}, \phi_{0}\right)$ y $d V_{0}=\xi_{0}^{2} \operatorname{sen} \theta_{0} d \xi_{0} d \theta_{0} d \phi_{0}$ 



\section{Solución numérica al problema de cerradura en la celda de Chang con geometría esférica}

En muchos problemas de ingeniería, resulta imposible obtener la solución analítica de problemas representados por ecuaciones diferenciales parciales, con el fin de evitar tal dificultad, y lograr resolver el problema con la ayuda de computadoras, es necesario presentar el problema de una manera puramente algebraica, esto es posible mediante el proceso de discretización, en donde el conjunto infinito de números que representan la función incógnita en el continuo es reemplazado por un número finito de parámetros incógnita, y este proceso requiere alguna forma de aproximación. Una de las formas más simples de discretización es mediante Métodos de Diferencias Finitas.

El método de diferencias finitas que se implementa en este trabajo para encontrar la solución numérica del problema de cerradura, es el método Implícito de Dirección Alternante conocido como ADI por sus siglas en inglés (Alternating Direction Implicit Method). Únicamente se trata el caso en el que el problema de cerradura es axialmente simétrico (independiente de la variable $\phi$ ), esto es, para la componente $z$ del vector de cerradura que permite determinar el coeficiente longitudinal de dispersión, en el que se implementará el método ADI-2D. Para calcular numéricamente los coeficientes de dispersión transversales, es necesario implementar el esquema ADI tridimensional, (ADI3D, (ver Apéndice A.7) para determinar las componentes $x$ y $y$ del vector de cerradura, cuyo problema de cerradura presenta funcionalidad en las tres coordenadas esféricas. Sin embargo, a pesar de haber llevado a cabo un intenso esfuerzo de cómputo, realizando un número importante de corridas del programa de este esquema numérico, para diferentes valores de los parámetros numéricos (paso de tiempo, número de nodos de los intervalos, tolerancia, condición inicial) no se obtuvo convergencia a una única función. Esto se debe a la condición de frontera de Neumann en el problema de cerradura que no permite conservar el orden de precisión del método ADI original. 


\subsection{Diferencias finitas}

Equation Chapter 6 Section 1

La aproximación de la primera y segunda derivada parcial en diferencias finitas está basada en la expansión en series de Taylor de orden uno y dos respectivamente, de la función a derivar.

Para un paso de tiempo $p$, los tres casos de discretización en diferencias finitas (hacia delante, hacia atrás y centrada) de la primer derivada parcial de $B_{\lambda}$ con respecto a $\xi$ pueden representarse por medio de la siguiente ecuación

$$
\left.\frac{\partial B_{\lambda}}{\partial \xi}\right|_{i, j, k} ^{p}=\frac{\alpha_{1} B_{\lambda i+1, j, k}^{p}+\alpha_{2} B_{\lambda i, j, k}^{p}+\alpha_{3} B_{\lambda i-1, j, k}^{p}}{\alpha_{4} \Delta \xi}
$$

El valor de los coeficientes en cada caso se presenta en la tabla siguiente

\begin{tabular}{|c|cccc|}
\hline Diferenciación finita & $\alpha_{1}$ & $\alpha_{2}$ & $\alpha_{3}$ & $\alpha_{4}$ \\
\hline Hacia delante & 1 & -1 & 0 & 1 \\
Hacia atrás & 0 & 1 & -1 & 1 \\
Centrada & 1 & -2 & 1 & 2 \\
\hline
\end{tabular}

Los errores de truncamiento en la aproximación de la primer derivada por diferencias finitas hacia delante y hacia atrás es del orden de $O[(\Delta \xi)]$, mientras que, para el caso de diferencias finitas centradas es de $O\left[(\Delta \xi)^{2}\right]$.

A un paso de tiempo $p$, la representación de la segunda derivada parcial de $B_{\lambda}$ con respecto a $\xi$ usando la discretización en diferencias finitas centradas, puede expresarse como

$$
\left.\frac{\partial^{2} B_{\lambda}}{\partial \xi^{2}}\right|_{i, j, k} ^{p}=\frac{B_{\lambda i+1, j, k}^{p}-2 B_{\lambda i, j, k}^{p}+B_{\lambda i-1, j, k}^{p}}{(\Delta \xi)^{2}}+O\left[(\Delta \xi)^{2}\right]
$$


El error de truncamiento en este caso es del orden de $O\left[(\Delta \xi)^{2}\right]$.

Las ecuaciones (6.1.1) y (6.1.2) son análogas para las primeras derivadas parciales en las direcciones $\theta$ y $\phi$.

En el método de diferencias finitas, para la discretización de la derivada temporal se hace uso de la de la aproximación de la primera derivada parcial hacia delante,

$$
\left.\frac{\partial B_{\lambda}}{\partial \tau}\right|_{i, j, k} ^{p}=\frac{B_{\lambda i, j, k}^{p+1}-B_{\lambda i, j, k}^{p}}{\Delta \tau}
$$

Las aproximaciones en diferencias finitas de las segundas derivadas parciales, que aparecen en la ecuación diferencial parcial a resolver se presentan a continuación

Dirección $\xi$

$$
\left.\frac{1}{\xi^{2}} \frac{\partial}{\partial \xi}\left(\xi^{2} \frac{\partial B_{\lambda}}{\partial \xi}\right)\right|_{i, j, k} ^{p}=\frac{1}{(\Delta \xi)^{2}}\left[\left(\frac{\xi_{i+\frac{1}{2}}}{\xi_{i}}\right)^{2}\left(B_{\lambda i+1, j, k}^{p}-B_{\lambda i, j, k}^{p}\right)-\left(\frac{\xi_{i-\frac{1}{2}}}{\xi_{i}}\right)^{2}\left(B_{\lambda i, j, k}^{p}-B_{\lambda i-1, j, k}^{p}\right)\right]
$$

Dirección $\theta$

$$
\begin{aligned}
& \left.\frac{1}{\xi^{2} \operatorname{sen} \theta} \frac{\partial}{\partial \theta}\left(\operatorname{sen} \theta \frac{\partial B_{\lambda}}{\partial \theta}\right)\right|_{i, j, k} ^{p}= \\
& \frac{1}{\xi_{i}^{2}(\Delta \theta)^{2}}\left[\left(\frac{\operatorname{sen} \theta_{j+\frac{1}{2}}}{\operatorname{sen} \theta_{j}}\right)\left(B_{\lambda i, j+1, k}^{p}-B_{\lambda i, j, k}^{p}\right)-\left(\frac{\operatorname{sen} \theta_{j-\frac{1}{2}}}{\operatorname{sen} \theta_{j}}\right)\left(B_{\lambda i, j, k}^{p}-B_{\lambda i, j-1, k}^{p}\right)\right]
\end{aligned}
$$

Dirección $\phi$ 


$$
\left.\frac{1}{\xi^{2} \operatorname{sen}^{2} \theta} \frac{\partial^{2} B_{\lambda}}{\partial \phi^{2}}\right|_{i, j, k} ^{p}=\frac{B_{\lambda i, j, k+1}^{p}-2 B_{\lambda i, j, k}^{p}+B_{\lambda i, j, k-1}^{p}}{\xi_{i}^{2} \operatorname{sen}^{2} \theta_{j}(\Delta \phi)^{2}}
$$

Para la obtención de las ecuaciones (6.1.4) y (6.1.5) se empleó la aproximación en diferencias finitas centradas para el primer derivada alrededor del nodo $i$ (nodo $j$ ), de acuerdo al siguiente esquema de discretización

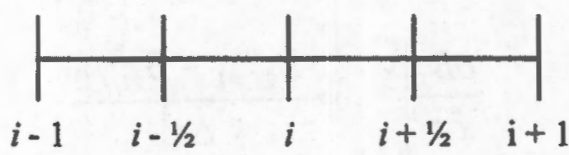

Figura 6.1 Esquema de discretización para la segunda derivada

El primer paso para resolver la ecuación diferencial parcial, por un método de diferencias finitas, consiste en discretizar el dominio continuo espacio-temporal, en el que dicha ecuación está definida. El problema de cerradura es un problema cuasi-estacionario y presenta simetría en la dirección $\phi$ (Apéndice A.4) en la celda de Chang con geometría esférica. Dada la simetría de dicho problema, es posible resolverlo únicamente en un hemisferio de la fase fluida de la celda esférica de Chang $V_{\gamma}{ }^{*}$. La descripción geométrica de esta región $V_{r}^{*} \subset \mathbb{R}^{3}$ es:

$$
V_{r}^{*}=\left\{(\xi, \theta, \phi) \mid \xi \in\left[\xi_{p}, \xi_{e}\right], \quad \theta \in[0, \pi], \phi \in[0, \pi]\right\}
$$

Para poder discretizar la región $V_{y}{ }^{*}$, es necesario particionar el intervalo de la coordenada radial $\left[\xi_{p}, \xi_{e}\right]$, el de la coordenada angular $[0, \pi]$ y de la coordenada azimutal $[0, \pi]$.

Sean

$P_{\xi}$ una partición regular de orden $N$ del intervalo radial $\left[1, \xi_{e}\right]$ 


$$
\begin{aligned}
P_{\xi} & =\left\{\xi_{p}=\xi_{1}, \xi_{2}, \ldots, \xi_{N-1}, \xi_{N}=\xi_{e}\right\} \\
& =\left\{\xi_{p}=\xi_{1}, \xi_{i}, \xi_{N} \mid \xi_{i}=\xi_{i-1}+\Delta \xi, \quad i=2, \ldots, N-1 ; \Delta \xi=\frac{\xi_{e}-\xi_{p}}{N-1}\right\}
\end{aligned}
$$

$P_{\theta}$ una partición regular de orden $M$ del intervalo azimutal $[0, \pi]$

$$
\begin{aligned}
P_{\theta} & =\left\{0=\theta_{1}, \theta_{2}, \ldots, \theta_{M-1}, \theta_{M}=\pi\right\} \\
& =\left\{0=\theta_{1}, \theta_{j}, \theta_{M}=\pi \mid \theta_{j}=\theta_{j-1}+\Delta \theta, j=2, \ldots, M-1 ; \Delta \theta=\frac{\pi}{M-1}\right\}
\end{aligned}
$$

$P_{\phi}$ una partición regular de orden S del intervalo azimutal $[0, \pi]$

$$
\begin{aligned}
P_{\phi} & =\left\{0=\phi_{1}, \phi_{2}, \ldots, \phi_{L-1}, \phi_{L}=\pi\right\} \\
& =\left\{0=\phi_{1}, \phi_{k}, \phi_{L}=\pi \mid \phi_{k}=\phi_{k-1}+\Delta \phi, k=2, \ldots, S-1 ; \Delta \phi=\frac{\pi}{S-1}\right\}
\end{aligned}
$$

De esta manera, a un paso de tiempo $p$, la componente $\lambda$ del vector de cerradura $B_{\lambda}(\xi, \theta, \phi)$ en un punto del dominio espacial discretizado $(i, j, k)$ puede ser reemplazada por $B_{\lambda}(i \Delta \xi, j \Delta \theta, k \Delta \phi)$ denotada por $B_{\lambda i, j, k}$.

De manera similar, a cualquier paso de tiempo $p$, las componentes del campo de velocidad $U_{\gamma_{\xi}}(\xi, \theta)$ y $U_{\gamma \theta}(\xi, \theta)$ en un punto del dominio discretizado $(i, j, k)$ están dadas por $U_{p \xi}(i \Delta \xi, j \Delta \theta)$ y $U_{p \theta}(i \Delta \xi, j \Delta \theta)$, denotadas por $U_{\xi}^{i, j}$ y $U_{\theta}^{i, j}$. La componente $\lambda$ del campo de desviación espacial del campo de velocidad $\tilde{U}_{p \lambda}(\xi, \theta)$, es denotada en el espacio discretizado como $\tilde{U}_{i, j}$. 


\subsection{ADI-2D}

Equation Chapter 6 Section 2

El método ADI fue sugerido por Douglas y Peaceman (1955), y Peaceman y Rachford (1955) para resolver la ecuación de calor en 2-D, y consiste en una modificación de la aproximación de Crank-Nicolson. En 1961, el método se extendió a problemas ligeramente no-lineales y problemas con tres variables espaciales. Este esquema de discretización, es incondicionalmente estable, sólo para problemas bidimensionales y presenta precisión de segundo orden en el espacio y en el tiempo, aunque frecuentemente ocasiona disipación del error, especialmente, en problemas dominados por convección (Karaa y Zhang 2004), mientras que para tres dimensiones es condicionalmente estable y tiene precisión de primer orden en el tiempo. Este método se ha aplicado a diferentes problemas físicos, como por ejemplo, para resolver la ecuación de convección-difusión (Chin et al., 1984), la ecuación de Schördinger (Dendy, 1977), la ecuación hiperbólica de conducción de calor (Jarzebski \& Thullie, 1986), sistemas de difusión-reacción (Chiu y Walkington, 1997), para predecir la temperatura de los chips en circuitos electrónicos (Wang et al., 2003).

Para encontrar la solución numérica de un problema de valores a la frontera en estado estacionario, el método ADI introduce un tiempo de marcha ficticio, volviendo dicho problema dependiente del tiempo. Así, la solución numérica del problema de contorno original, es la solución en estado estacionario del problema ficticio dependiente del tiempo. $\mathrm{El}$ método $\mathrm{ADI}$, es un esquema numérico que, reduce un problema en dos dimensiones, en una sucesión de dos problemas unidimensionales, particionando el tiempo de marcha de $p$ a $p+1$ en dos pasos: uno de $p$ a $p+1 / 2$ y el otro de $p+1 / 2$ a $p+1$. En la Figura 6.2 se muestra que, en el paso I, la dirección $\xi$ es implícita basándose en el método de Crank-Nicolson, mientras que la dirección $\theta$ es explícita. Para cada nodo $j$ de la malla, hay $M-2$ ecuaciones correspondientes al punto $(i, j)$, las otras dos ecuaciones se determinan de las condiciones de frontera. Como cada punto $(i, j)$ está relacionado a los puntos $(i-1, j)$ y $(i+1, j)$, la matriz de coeficientes es una matriz tridiagonal que puede invertirse usando el algoritmo de Thomas. En el paso II, la dirección $\theta$ es implícita y la dirección $\xi$ es explicita de manera análoga al paso $I$. 


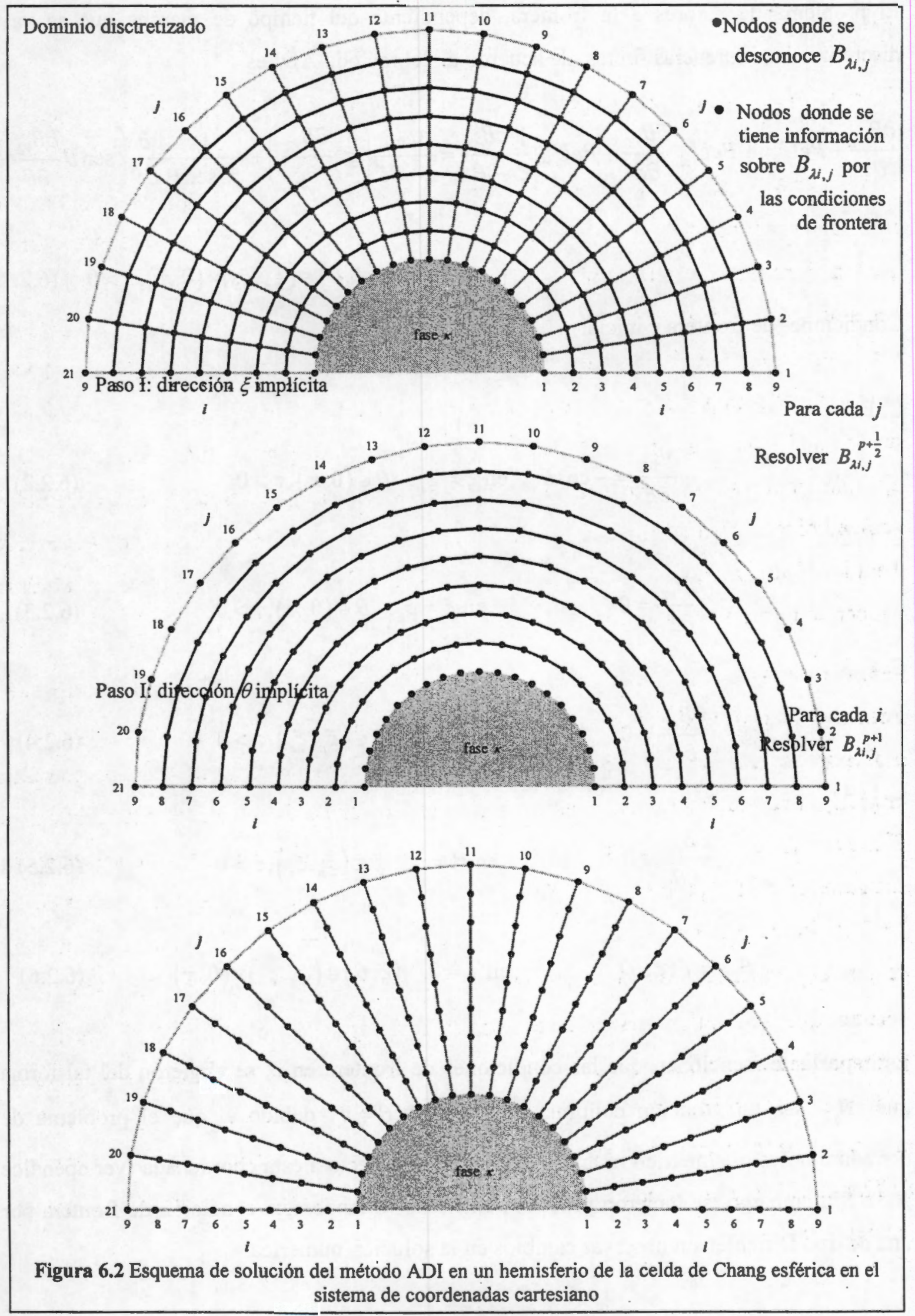


El problema de valores a la frontera, dependiente del tiempo de marcha que se va a discretizar en diferencias finitas, de acuerdo al del método ADI es

$$
\begin{array}{r}
\frac{\partial B_{y z}}{\partial \tau}+P e \tilde{U}_{\gamma z}+P e U_{\gamma \xi} \frac{\partial B_{y z}}{\partial \xi}+P e U_{\gamma \theta} \frac{1}{\xi} \frac{\partial B_{\gamma z}}{\partial \theta}=\frac{1}{\xi^{2}} \frac{\partial}{\partial \xi}\left(\xi^{2} \frac{\partial B_{y z}}{\partial \xi}\right)+\frac{1}{\xi^{2} \operatorname{sen} \theta} \frac{\partial}{\partial \theta}\left(\operatorname{sen} \theta \frac{\partial B_{\gamma z}}{\partial \theta}\right) \\
(\xi, \theta) \in\left(\xi_{p}, \xi_{e}\right) \times(0, \pi), \tau>0
\end{array}
$$

Condiciones de frontera e inicial

C. F. 1

$$
\frac{\partial B_{y z}}{\partial \xi}=-\cos \theta \quad \text { en } \xi=\xi_{p}, \quad \theta \in(0, \pi), \tau>0
$$

C. F. 2

$$
\frac{\partial B_{y z}}{\partial \xi}=0 \quad \text { en } \xi=\xi_{e}, \theta \in(0, \pi), \tau>0
$$

C. F. 3

$$
\frac{\partial B_{y z}}{\partial \theta}=0 \quad \text { en } \theta=0, \xi \in\left(\xi_{p}, \xi_{e}\right), \tau>0
$$

C. F. 4

$$
\frac{\partial B_{y z}}{\partial \theta}=0 \quad \text { en } \theta=\pi, \xi \in\left(\xi_{p}, \xi_{e}\right), \tau>0
$$

C. I.

$$
B_{y z}=f(\xi, \theta) \quad \text { en } \tau=0,(\xi, \theta) \in\left(\xi_{p}, \xi_{e}\right) \times(0, \pi)
$$

Es importante mencionar, que las condiciones de frontera en $\theta$, se eligieron del tal forma que, $B_{y z}$ sea una función continua en $\theta=0$ y $\theta=\pi$, debido a, que el problema de cerradura no es ni simétrico ni anti-simétrico con respecto a esta coordenada (ver apéndice A.5). Sin embargo, se hicieron pruebas numéricas variando esta condición de frontera por una de tipo Dirichlet sin observar cambios en la solución numérica. 
Las ecuaciones discretizadas que definen cada uno de los pasos del método $\mathrm{ADI}$, del problema de cerradura dependiente del tiempo de marcha (6.2.2)-(6.2.6) a resolver son:

Paso I

$$
\begin{aligned}
{\left[\frac{\partial B_{y z}}{\partial \tau}\right]_{i, j}^{p+\frac{1}{2}} } & =\left[\frac{1}{\xi^{2}} \frac{\partial}{\partial \xi}\left(\xi^{2} \frac{\partial B_{y z}}{\partial \xi}\right)-P e U_{r \xi} \frac{\partial B_{y z}}{\partial \xi}-P e \tilde{U}_{y z}\right]_{i, j}^{p+\frac{1}{2}}+ \\
& +\left[\frac{1}{\xi^{2} \operatorname{sen} \theta} \frac{\partial}{\partial \theta}\left(\operatorname{sen} \theta \frac{\partial B_{y z}}{\partial \theta}\right)-P e U_{\gamma \theta} \frac{1}{\xi} \frac{\partial B_{y z}}{\partial \theta}\right]_{i, j}^{p}
\end{aligned}
$$

Paso II

$$
\begin{aligned}
{\left[\frac{\partial B_{y z}}{\partial \tau}\right]_{i, j}^{p+1} } & =\left[\frac{1}{\xi^{2}} \frac{\partial}{\partial \xi}\left(\xi^{2} \frac{\partial B_{r z}}{\partial \xi}\right)-P e U_{r \xi} \frac{\partial B_{r \xi}}{\partial \xi}-P e \tilde{U}_{r z}\right]_{i, j}^{p+\frac{1}{2}}+ \\
& +\left[\frac{1}{\xi^{2} \operatorname{sen} \theta} \frac{\partial}{\partial \theta}\left(\operatorname{sen} \theta \frac{\partial B_{r z}}{\partial \theta}\right)-P e U_{r \theta} \frac{1}{\xi} \frac{\partial B_{y z}}{\partial \theta}\right]_{i, j}^{p+1}
\end{aligned}
$$

De acuerdo a la aproximación en diferencias finitas, para la primer derivada dada por la ecuación (6.1.1), se tiene que la discretización de las condiciones de frontera e inicial están dadas por

C. F. 1: $\quad \frac{B_{\lambda 2, j, k}^{p}-B_{\lambda 1, j, k}^{p}}{\Delta \xi}=-\cos \theta_{j} \quad j=2, \ldots, N-1, k=2, \ldots, S-1, p>1$

C. F. 2: $\quad \frac{B_{\lambda M, j, k}^{p}-B_{\lambda M-1, j, k}^{p}}{\Delta \xi}=0 \quad j=2, \ldots, N-1, k=2, \ldots, S-1, p>1$

C. F. 3: $\quad \frac{B_{\lambda i, 2, k}^{p}-B_{2 i, 1, k}^{p}}{\Delta \theta}=0 \quad i=2, \ldots, M-1, k=2, \ldots, S-1, p>1$

C. F. 4: $\quad \frac{B_{\lambda i, N, k}^{p}-B_{Z i, N-1, k}^{p}}{\Delta \theta}=0 \quad i=2, \ldots, M-1, k=2, \ldots, S-1, p>1$

C. I.:

$$
B_{\lambda i, j, k}^{1}=f_{i, j} \quad i=2, \ldots, M-1, j=2, \ldots N-1, k=2, \ldots, S-1
$$


Para simplificar las ecuaciones discretizadas de cada uno de los pasos, es necesario definir las siguientes identidades:

Parámetros numéricos

$$
\begin{gathered}
r_{1}^{\xi}=\frac{\Delta \tau}{\Delta \xi} \\
r_{2}^{\xi}=\frac{\Delta \tau}{(\Delta \xi)^{2}} \\
r_{1}^{\theta}=\frac{\Delta \tau}{\Delta \theta} \\
r_{2}^{\theta}=\frac{\Delta \tau}{(\Delta \theta)^{2}} \\
r_{2}^{\theta}=\frac{\Delta \tau}{(\Delta \phi)^{2}}
\end{gathered}
$$

Coeficientes numéricos

$$
\begin{gathered}
A_{i, j}^{\xi}=r_{2}^{\xi}\left(\frac{\xi_{i-\frac{1}{2}}}{\xi_{i}}\right)^{2}-r_{1}^{\xi} P e\left(\frac{\alpha_{3}^{\xi}}{\alpha_{4}^{\xi}}\right) U_{\xi}^{i, j} \\
B_{i, j}^{\xi}=r_{2}^{\xi}\left[\left(\frac{\xi_{i+\frac{1}{2}}}{\xi_{i}}\right)^{2}+\left(\frac{\xi_{i-\frac{1}{2}}}{\xi_{i}}\right)^{2}\right]+r_{1}^{\xi} P e\left(\frac{\alpha_{2}^{\xi}}{\alpha_{4}^{\xi}}\right) U_{\xi}^{i, j} \\
C_{i, j}^{\xi}=r_{2}^{\xi}\left(\frac{\xi_{i+\frac{1}{2}}}{\xi_{i}}\right)^{2}-r_{1}^{\xi} P e\left(\frac{\alpha_{1}^{\xi}}{\alpha_{4}^{\xi}}\right) U_{\xi}^{i, j} \\
A_{i, j}^{\theta}=\frac{r_{2}^{\theta}}{\xi_{i}^{2}}\left(\frac{\operatorname{sen} \theta}{\operatorname{sen} \theta_{j}}\right)-\frac{r_{1}^{\theta}}{\xi_{i}} P e\left(\frac{\alpha_{3}^{\theta}}{\alpha_{4}^{\theta}}\right) U_{\theta}^{i, j}
\end{gathered}
$$




$$
\begin{gathered}
B_{i, j}^{\theta}=\frac{r_{2}^{\theta}}{\xi_{i}^{2}}\left[\frac{\operatorname{sen} \theta_{j+\frac{1}{2}}}{\operatorname{sen} \theta_{j}}+\frac{\operatorname{sen} \theta_{j-\frac{1}{2}}}{\operatorname{sen} \theta_{j}}\right]+\frac{r_{i}^{\theta}}{\xi_{i}} P e\left(\frac{\alpha_{2}^{\theta}}{\alpha_{4}^{\theta}}\right) U_{\theta}^{i, j} \\
C_{i, j}^{\theta}=\frac{r_{2}^{\theta}}{\xi_{i}^{2}} \frac{\operatorname{sen} \theta_{j+\frac{1}{2}}}{\operatorname{sen} \theta_{j}}-\frac{r_{1}^{\theta}}{\xi_{i}} P e\left(\frac{\alpha_{1}^{\theta}}{\alpha_{4}^{\theta}}\right) U_{\theta}^{i, j} \\
A_{i, j}^{\theta}=\frac{r_{2}^{\theta}}{\xi_{i}^{2} \operatorname{sen}^{2} \theta_{j}}
\end{gathered}
$$

Después de aplicar las aproximaciones en diferencias finitas de la primera y segunda derivada, dadas por las ecuaciones (6.1.3)-(6.1.5), a las ecuaciones (6.2.7)-(6.2.8), que definen los pasos del método $\mathrm{ADI}$, multiplicando las ecuaciones resultantes por $\Delta \tau / 2$, factorizando, identificando los parámetros numéricos definidos por las ecuaciones (6.2.14), y usando la definición de los coeficientes numéricos dados por las ecuaciones (6.2.19)(6.2.24) (para más detalle, ver el apéndice A.6), resulta que las ecuaciones en cada uno de los pasos son

\section{Paso I}

Para cada valor de $j$ y $p$, la ecuación del paso I, representa un sistema tridiagonal de ecuaciones lineales para los valores de $B_{z i-1, j}^{p+\frac{1}{2}}, B_{z i, j}^{p+\frac{1}{2}}$ y $B_{z i+1, j}^{p+\frac{1}{2}}$.

Las ecuaciones del paso I para cada nodo son las siguientes:

Para el nodo $i=2, j=1,2, \ldots, M$ y $p=1,2, \ldots$ y de la condición de frontera, dada por la ecuación (6.2.9), se tiene que

$$
\begin{aligned}
\left(1-A_{2, j}^{\xi}+B b_{2, j}^{\xi}\right) B_{z 2, j}^{p+\frac{1}{2}}-C_{i, j}^{\xi} B_{z 3, j}^{p+\frac{1}{2}}=-P e \frac{\Delta \tau}{2} \tilde{U}_{\xi}^{2, j}+ & A_{2, j}^{\theta} B_{z 2, j-1}^{p}+\left(1-B b_{2, j}^{\theta}\right) B_{z 2, j}^{p}(6.2 \\
& +C_{i, j}^{\theta} B_{z 2, j+1}^{p}+A_{2, j}^{\xi} \Delta \xi \cos \theta_{j}
\end{aligned}
$$

Para los nodos $i=3,4, \ldots, N-2, j=1,2, \ldots, M$ y $p=1,2, \ldots$ 


$$
\begin{array}{r}
-A_{i, j}^{\xi} B_{z i-1, j}^{p+\frac{1}{2}}+\left(1+B b_{i, j}^{\xi}\right) B_{z i, j}^{p+\frac{1}{2}}-C_{i, j}^{\xi} B_{z i+1, j}^{p+\frac{1}{2}}=-P e \frac{\Delta \tau}{2} \tilde{U}_{z}^{i, j}+A_{i, j}^{\theta} B_{z i, j-1}^{p}+\left(1-B b_{i, j}^{\theta}\right) B_{z i, j}^{p} \\
+C_{i, j}^{\theta} B_{z i, j+1}^{p}
\end{array}
$$

Para el nodo $i=N-1, j=1,2, \ldots, M$ y $p=1,2, \ldots$ y de la condición de frontera, dada por la ecuación (6.2.10), se obtiene que

$$
\begin{aligned}
-A_{N-1, j}^{\xi} B_{Z N-2, j}^{p+\frac{1}{2}}+\left(1+B b_{N-1, j}^{\xi}-C_{N-1, j}^{\xi}\right) B_{Z N-1, j}^{p+\frac{1}{2}} & =-P e \frac{\Delta \tau}{2} \tilde{U}_{Z}^{N-1, j}+A_{N-1, j}^{\theta} B_{Z N-1, j-1}^{p} \\
& +\left(1-B b_{N-1, j}^{\theta}\right) B_{Z N-1, j}^{p}+C_{N-1, j}^{\theta} B_{Z N-1, j+1}^{p}
\end{aligned}
$$

El sistema de ecuaciones dado por las ecuaciones (6.2.26)-(6.2.28) toma la siguiente representación matricial

$$
\left(\begin{array}{ccccc}
1+B_{2, j}^{k}-A_{2, j}^{k} & -C_{2,}^{k} & 0 & \cdots & 0 \\
-A_{3, j}^{t} & 1+B_{3, j}^{t} & -C_{3, j}^{k} & \ddots & \vdots \\
0 & \ddots & \ddots & \ddots & 0 \\
\vdots & \ddots & -A_{N-2,}^{k} & 1+B_{N-2,}^{k} & -C_{N-2, j}^{k} \\
0 & \cdots & 0 & -A_{N-1, j}^{k} & 1+B_{N-1, j}^{k}
\end{array}\right)\left(\begin{array}{c}
B_{22, j}^{p+\frac{1}{2}} \\
B_{23, j}^{p+\frac{1}{2}} \\
\vdots \\
B_{2 N-2, j}^{p+\frac{1}{2}} \\
B_{\lambda-1, j}^{p+\frac{1}{2}}
\end{array}\right)=\left(\begin{array}{c}
D_{\xi 2, j}^{p}+A_{2, j}^{k} \Delta \xi \cos \theta_{j} \\
D_{\xi 3, j}^{p} \\
\vdots \\
D_{\xi N-2, j}^{p} \\
D_{\xi N-1, j}^{p}
\end{array}\right)
$$

obien

$$
\mathbf{A}_{\xi} \mathbf{R}^{\prime}=\mathbf{D}_{\theta}^{\prime}
$$

Donde

$$
\begin{aligned}
& D_{\theta 2, j}^{I}=-P e \frac{\Delta \tau}{2} \tilde{U}_{\xi}^{2, j}+A_{2, j}^{\theta} B_{z 2, j-1}^{p}+\left(1-B b_{2, j}^{\theta}\right) B_{Z 2, j}^{p}+C_{i, j}^{\theta} B_{z 2, j+1}^{p}+A_{2, j}^{\xi} \Delta \xi \cos \theta_{j} \\
& D_{\theta i, j}^{I}=-P e \frac{\Delta \tau}{2} \tilde{U}_{z}^{i, j}+A_{i, j}^{\theta} B_{z i, j-1}^{p}+\left(1-B b_{i, j}^{\theta}\right) B_{Z i, j}^{p}+C_{i, j}^{\theta} B_{Z i, j+1}^{p} \\
& D_{\theta N, j}^{I}=-P e \frac{\Delta \tau}{2} \tilde{U}_{z}^{N-1, j}+A_{N-1, j}^{\theta} B_{Z N-1, j-1}^{p}+\left(1-B b_{N-1, j}^{\theta}\right) B_{Z N-1, j}^{p}+C_{N-1, j}^{\theta} B_{Z N-1, j+1}^{p}
\end{aligned}
$$

Paso II 
Para cada valor de $i$ y $p$, la ecuación del paso II, representa un sistema tridiagonal de ecuaciones lineales para los valores de $B_{Z i, j-1}^{p+1}, B_{Z i, j}^{p+1}$ y $B_{Z i, j+1}^{p+1}$.

Las ecuaciones del paso II para cada nodo son las siguientes:

Para el nodo $j=2, i=1,2, \ldots, N$ y $p=1,2, \ldots$ y de la condición de frontera dada por la ecuación (6.2.11), se tiene que

$$
\begin{array}{r}
\left(1+B b_{i, 2}^{\theta}-A_{i, 2}^{\theta}\right) B_{z i, 2}^{p+1}-C_{i, 2}^{\theta} B_{z i, 3}^{p+1}=-P e \frac{\Delta \tau}{2} \tilde{U}_{z}^{i, 2}+A_{i, 2}^{\xi} B_{z i-1,2}^{p+\frac{1}{2}}+\left(1-B b_{i, j}^{\xi}\right) B_{z i, 2}^{p+\frac{1}{2}} \\
+C_{i, j}^{\xi} B_{z i+1,2}^{p+\frac{1}{2}}
\end{array}
$$

Para los nodos $i=1,2, \ldots, N-2, j=3,4, \ldots, M$ y $p=1,2, \ldots$

$$
\begin{array}{r}
-A_{i, j}^{\theta} B_{z i, j-1}^{p+1}+\left(1+B b_{i, j}^{\theta}\right) B_{z i, j}^{p+1}-C_{i, j}^{\theta} B_{z i, j+1}^{p+1}=-P e \frac{\Delta \tau}{2} \tilde{U}_{z}^{i, j}+A_{i, j}^{\xi} B_{z i-1, j}^{p+\frac{1}{2}}+\left(1-B b_{i, j}^{\xi}\right) B_{z i, j}^{p+\frac{1}{2}} \\
+C_{i, j}^{\xi} B_{z i+1, j}^{p+\frac{1}{2}}
\end{array}
$$

Para el nodo $j=M-1, i=1,2, \ldots, N$ y $p=1,2, \ldots$ y de la condición de frontera, dada por la ecuación (6.2.12), se obtiene que

$$
\begin{aligned}
-A_{i, M-1}^{\theta} B_{z i, M-2}^{p+1}+\left(1+B b_{i, M-1}^{\theta}-C_{i, M-1}^{\theta}\right) B_{z i, M-1}^{p+1} & =-P e \frac{\Delta \tau}{2} \tilde{U}_{z}^{i, M-1}+A_{i, M-1}^{\xi} B_{Z i-1, M-1}^{p+\frac{1}{2}} \\
+ & \left(1-B b_{i, j}^{\xi}\right) B_{Z i, M-1}^{p+\frac{1}{2}}+C_{i, M-1}^{\xi} B_{Z i+1, M-1}^{p+\frac{1}{2}}
\end{aligned}
$$

El sistema de ecuaciones dado por las expresiones (6.2.31)-(6.2.32) toma la siguiente representación matricial 


$$
\left(\begin{array}{ccccc}
1+B_{2, j}^{\theta}-A_{2, j}^{\theta} & -C_{2, j}^{\theta} & 0 & \cdots & 0 \\
-A_{i, 3}^{\theta} & 1+B_{i, 3}^{\theta} & -C_{i, 3}^{\theta} & \ddots & \vdots \\
0 & \ddots & \ddots & \ddots & 0 \\
\vdots & \ddots & -A_{i, M-2}^{\theta} & 1+B_{i, M-2}^{\theta} & -C_{i, M-2}^{\theta} \\
0 & \cdots & 0 & -A_{i, M-1}^{\theta} & 1+B_{i, M-1}^{\theta}-C_{i, M-1}^{\theta}
\end{array}\right)\left(\begin{array}{c}
B_{2, i}^{p+1} \\
B_{2 i, 3}^{p+3} \\
\vdots \\
B_{2 i, M-2}^{p+1} \\
B_{z i, M-1}^{p+1}
\end{array}\right)=\left(\begin{array}{c}
D_{\xi i, 2}^{p+\frac{1}{2}} \\
D_{\xi i, 3}^{p+\frac{1}{2}} \\
\vdots \\
\\
D_{\xi i, M-2}^{p+\frac{1}{2}} \\
D_{\xi, M-1}^{p+\frac{1}{2}}
\end{array}\right)
$$

obien

$$
\mathbf{A}_{\theta} \mathbf{R}^{\prime \prime}=\mathrm{D}_{\xi}^{\prime \prime}
$$

Donde

$$
\begin{aligned}
D_{\xi i, 2}^{I I} & =-P e \frac{\Delta \tau}{2} \tilde{U}_{2}^{i, 2}+A_{i, 2}^{\xi} B_{Z i-1,2}^{p+\frac{1}{2}}+\left(1-B b_{i, j}^{\xi}\right) B_{z i, 2}^{p+\frac{1}{2}}+C_{i, j}^{\xi} B_{z i+1,2}^{p+\frac{1}{2}} \\
D_{\xi i, 3}^{I I} & =-P e \frac{\Delta \tau}{2} \tilde{U}_{2}^{i, j}+A_{i, j}^{\xi} B_{z i-1, j}^{p+\frac{1}{2}}+\left(1-B b_{i, j}^{\xi}\right) B_{z i, j}^{p+\frac{1}{2}}+C_{i, j}^{\xi} B_{z i+1, j}^{p+\frac{1}{2}} \\
D_{\xi i, M-1}^{I I} & =-P e \frac{\Delta \tau}{2} \tilde{U}_{2}^{i, M-1}+A_{i, M-1}^{\xi} B_{Z i-1, M-1}^{p+\frac{1}{2}}+\left(1-B b_{i, j}^{\xi}\right) B_{z i, M-1}^{p+\frac{1}{2}}+C_{i, M-1}^{\xi} B_{2 i+1, M-1}^{p+\frac{1}{2}}
\end{aligned}
$$




\subsection{Algoritmo numérico}

\section{Datos}

Extremos de los intervalos de las componentes $\xi$ y $\theta$.

$N$ : Número de nodos en el intervalo radial $\xi$.

M: Número de nodos en el intervalo angular $\theta$.

$\Delta \tau$. Paso de tiempo.

$\tau_{\max }$ : Tiempo máximo.

$P e$ : Número de Péclet.

$\varepsilon$ : Error de tolerancia.

\section{Calcular vectores de posición y parámetros numéricos}

Tamaños de paso de los intervalos del dominio espacial, $\Delta \xi, \Delta \theta$.

Generar los vectores de posición $\xi$ y $\theta$ de dimensiones $N$ y $M$.

Parámetros numéricos $r_{1}^{\xi}, r_{2}^{\xi}, r_{1}^{\theta}, r_{2}^{\theta}$ dados por las ecuaciones (6.2.14)-(6.2.18).

\section{Calcular}

Componentes del campo bidimensional de velocidad $U_{\xi}^{i, j}$ y $U_{\theta}^{i, j}$ dadas por las ecuaciones (5.3.1) y (5.3.2) respectivamente.

Componentes del campo bidimensional de velocidad $\left\langle U_{r^{\xi}}\right\rangle^{\gamma}$ y $\left\langle U_{r}\right\rangle^{\gamma}$ dadas por las ecuaciones (5.3.4) y (5.3.5) respectivamente.

Componente $Z$ del campo de desviación espacial del campo de velocidad $\tilde{U}_{i, j}$ dada por la ecuación (5.3.10).

Calcular la función de Green del problema de cerradura axialmente simétrico dada por la ecuación (4.6.12). 
Calcular la solución analítica $B_{\lambda i, j}^{1}$, del problema de cerradura dada por la ecuación (4.6.11) para después usarla como condición inicial en el método ADI.

\section{Calcular}

Calcular los coeficientes $A_{i, j}^{\xi}, B_{i, j}^{\xi}, C_{i, j}^{\xi}, A_{i, j}^{\theta}, B_{i, j}^{\theta}$ y $C_{i, j}^{\theta}$, dados por las ecuaciones (6.2.19)-(6.2.25)

\section{Ciclo de iteraciones}

Asignar al instante $\tau=0$ el paso de tiempo $p=1$

Asignar $B_{z i, j}^{1} \leftarrow B_{z}\left(\xi_{i}, \theta_{j}\right) \quad i=2, \ldots, N-1, j=2, \ldots, M-1$

\section{Paso I}

Encontrar las incógnitas $B_{z i-1, j}^{p+\frac{1}{2}}, \quad B_{z i, j}^{p+\frac{1}{2}}, \quad B_{Z i+1, j}^{p+\frac{1}{2}}$ para cada $j=2,3, \ldots, M-1$

For $\quad j=2, M-1$

Calcular

Las componentes del vector resultante $\mathbf{D}_{\theta}^{I}$ para $j=2, \ldots, M-1$ dadas por la ecuación (6.2.30).

Las componentes de la matriz tridiagonal de coeficientes $\mathbf{A}_{\xi}$, ver ecuación (6.2.29), generada por las siguientes diagonales de $N-2$ componentes

$$
\begin{aligned}
& \mathbf{A}_{\xi}^{1}=\left(\begin{array}{llllll}
0 & \ldots & -A_{i, j}^{\xi} & \ldots & -A_{N-1, j}^{\xi}
\end{array}\right), 3 \leq i \leq N-2 \text { y } 2 \leq j \leq M-1 \\
& \mathbf{B}_{\xi}^{1}=\left(\begin{array}{llllll}
1+B_{2, j}^{\xi}-A_{2, j}^{\xi} & \ldots & 1+B_{i, j}^{\xi} & \ldots & 1+B_{N-1, j}^{\xi}
\end{array}\right), 3 \leq i \leq N-2 \text { y } 2 \leq j \leq M-1 \\
& \mathbf{C}_{\xi}^{1}=\left(\begin{array}{llllll}
0 & \ldots & -C_{i, j}^{\xi} & \ldots & 0
\end{array}\right), 2 \leq i \leq N-1 \text { y } 2 \leq j \leq M-1
\end{aligned}
$$


Llamar la rutina con el algoritmo de Thomas

tridag $\left(\mathbf{A}_{\xi}^{l}, \mathbf{B}_{\xi}^{I}, \mathbf{C}_{\xi}^{l}, \mathbf{D}_{\xi}^{I}, \mathbf{R}^{I}, N-2\right)$.

Asignar $B_{z i, j}^{p+\frac{1}{2}} \leftarrow R_{i}^{l}, i=1, N-2$.

Asignar las condiciones de frontera.

End

\section{Paso II}

Encontrar las incógnitas $B_{z i, j-1}^{p+1}, \quad B_{Z i, j}^{p+1}, \quad B_{z i, j+1}^{p+1}$ para cada $i=2,3, \ldots, N-1$

For $\quad i=2, N-1$

Calcular

Las componentes del vector resultante $\mathbf{D}_{\xi}^{I l}$ para $i=2, \ldots, N-1$ dadas por la ecuación (6.2.35).

Las componentes de la matriz tridiagonal de coeficientes $\mathbf{A}_{\boldsymbol{\xi}}$, ver ecuación (6.2.34), generada por las diagonales dadas por los vectores de $M-2$ componentes dados por

$$
\begin{aligned}
& \mathbf{A}_{\theta}^{I I}=\left(\begin{array}{lllll}
0 & \ldots & -A_{i, j}^{\theta} & \ldots & -A_{i, M-1}^{\theta}
\end{array}\right), 2 \leq i \leq N-1 \text { y } 3 \leq j \leq M-2 \\
& \mathbf{B}_{\theta}^{I I}=\left(\begin{array}{llllll}
1+B_{2, j}^{\theta}-A_{2, j}^{\theta} & \ldots & 1+B_{i, j}^{\theta} & \ldots & 1+B_{i, M-1}^{\theta}
\end{array}\right), 2 \leq i \leq N-1 \text { y } 3 \leq j \leq M-2 \\
& \mathbf{C}_{\xi}^{I I}=\left(\begin{array}{llllll}
0 & \ldots & -C_{i, j}^{\theta} & \ldots & -C_{i, M-1}^{\theta}
\end{array}\right), 2 \leq i \leq N-1 \text { y } 2 \leq j \leq M-2
\end{aligned}
$$

Llamar la rutina con el algoritmo de Thomas tridag $\left(\mathbf{A}_{\theta}^{I I}, \mathbf{B}_{\theta}^{I I}, \mathbf{C}_{\theta}^{I l}, \mathbf{D}_{\theta}^{I I}, \mathbf{R}^{I I}, M-2\right)$. Asignar $B_{z i, j}^{p+\frac{1}{2}} \leftarrow R_{i}^{I}, i=1, N-2$.

Asignar las condiciones de frontera.

End 


\section{Calcular los coeficientes de medio efectivo}

Calcular el coeficiente de dispersión total

$$
\frac{D_{\gamma z z}^{*}}{\mathscr{D}_{\gamma}}=\frac{D_{e f f}^{z z}}{\mathscr{D}_{\gamma}}+\frac{D_{\gamma}^{z}}{\mathscr{D}_{\gamma}}
$$

\section{Comparar}

$$
\begin{aligned}
\text { Calcular error }= & \left|\left(\frac{D_{r z z}^{*}}{\mathscr{D}_{\gamma}}\right)_{\text {viejo }}-\left(\frac{D_{r z}^{*}}{\mathscr{D}_{\gamma}}\right)_{\text {muevo }}\right| \\
\mathrm{Si} \quad & \text { error } \geq \varepsilon \\
& \text { Revisar si } \tau \text { alcanzo } \tau_{\max } \\
& p \leftarrow p+1, \tau \leftarrow p \Delta \tau \\
& \text { Regresar al paso } 4 \\
\text { Sino } & \\
& \text { Imprimir la solución de la EDP en estado estacionario } \\
& \text { Fin del programa }
\end{aligned}
$$

Fin 


\section{Resultados}

En esta capítulo se presentan los resultados del coeficiente de dispersión longitudinal (dirección paralela a la dirección principal de la velocidad), obtenidos analítica y numéricamente; y del coeficiente de dispersión transversal (dirección ortogonal a la dirección principal de la velocidad) analíticos, que mejor se ajustan a resultados teóricos y experimentales previos, para diferentes valores del número de Péclet.

\subsection{Coeficiente longitudinal de dispersión total}

Equation Chapter 7 Section 1

En este trabajo se usa la celda de Chang con geometría esférica (Figura 7.1) para modelar un medio poroso homogéneo espacialmente periódico. El coeficiente de dispersión longitudinal en esta celda unitaria, corresponde a la componente $z z$ del tensor de dispersión total. En la sección 4.4 se demostró que, si el problema de cerradura para una componente dada del vector de cerradura es axialmente simétrico, es decir, que las condiciones

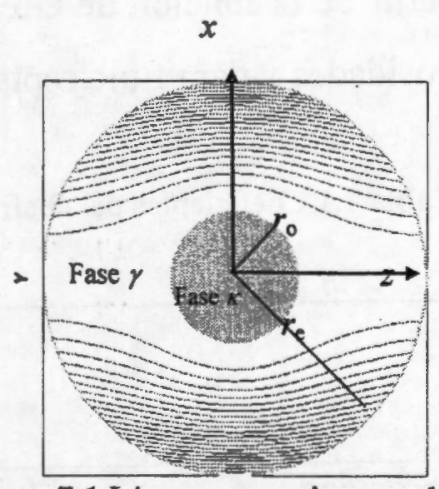

Figura 7.1 Líneas ue orriente en la celda de Chang de frontera y el término no homogéneo de la ecuación diferencial parcial no presentan dependencia la coordenada $\phi$, la solución a dicho problema es también axialmente simétrica y está dada por la ecuación (4.6.11). Para la componente $z$, el problema de cerradura es axialmente simétrico y por consiguiente la componente $z$ del vector de cerradura también lo es.

En la sección 4.5, se encontró la expresión de las componentes del tensor de dispersión total dada por la ecuación (4.7.2), que corresponde a un polinomio de grado dos en el número de Péclet, el caso de la componente $z z$, esta expresión se reduce a 
Para el caso del coeficiente longitudinal en la celda esférica de Chang, el coeficiente que acompaña al término $P e$ (ver ecuación (4.7.2)), es nulo, lo que hace que, la expresión del coeficiente longitudinal de dispersión se simplifique a

$$
\frac{D_{r z}^{*}}{\mathscr{D}_{p}}=1+A_{1}+A_{2} P e_{p}^{2}
$$

Para el cálculo de los coeficientes $A_{1}$ y $A_{2}$ se programó la función de Green, que es una serie infinita dada por la ecuación (4.6.12), las integrales dobles y simples involucradas en la determinación de estos coeficientes se realizaron numéricamente, basándose en el algoritmo de Simpson para integrales dobles y en el de Simpson compuesto respectivamente. En la Tabla 7.1 se presenta el valor de los coeficientes $A_{1}$ y $A_{2}$, calculados a partir de la función de Green, para el número de Péclet de la partícula $\left(P e_{p}\right)$ a diferentes porosidades y para flujo reptante y potencial.

Tabla 7.1 Coeficientes del coeficiente de dispersión longitudinal para el número de Péclet de la partícula $\left(P e_{p}\right)$ para flujo potencial y reptante

\begin{tabular}{|c|c|c|c|c|}
\hline \multicolumn{5}{|c|}{$\frac{D_{Y z}^{*}}{\mathscr{D}}=1+A_{1}+A_{2} P e_{p}^{2}$} \\
\hline Porosidad & \multicolumn{5}{|c|}{ Flujo potencial } & \multicolumn{2}{c|}{ Flujo reptante } \\
\cline { 2 - 5 }$\varepsilon_{\gamma}$ & $A_{2}$ & $A_{1}$ & $A_{2}$ & $A_{1}$ \\
\hline 0.1 & 5.5899 & 0.0153 & $2.413 \cdot 10^{-3}$ & 0.0153 \\
\hline 0.2 & $1.988 \cdot 10^{-1}$ & 0.0714 & $2.055 \cdot 10^{-3}$ & 0.0714 \\
\hline 0.3 & $1.915 \cdot 10^{-3}$ & 0.1905 & $1.708 \cdot 10^{-3}$ & 0.1905 \\
\hline 0.4 & $5.515 \cdot 10^{-3}$ & 0.4103 & $1.374 \cdot 10^{-3}$ & 0.4103 \\
\hline 0.5 & $1.034 \cdot 10^{-2}$ & 0.8000 & $1.057 \cdot 10^{-3}$ & 0.8000 \\
\hline 0.6 & $9.067 \cdot 10^{-3}$ & 1.5000 & $7.633 \cdot 10^{-4}$ & 1.5000 \\
\hline 0.7 & $5.605 \cdot 10^{-3}$ & 2.8406 & $4.990 \cdot 10^{-4}$ & 2.8406 \\
\hline 0.8 & $2.444 \cdot 10^{-3}$ & 5.8182 & $2.733 \cdot 10^{-4}$ & 5.8182 \\
\hline 0.9 & $5.577 \cdot 10^{-4}$ & 15.4286 & $9.897 \cdot 10^{-5}$ & 15.4286 \\
\hline
\end{tabular}


En la Figura 7.2 se presentan los perfiles del coeficiente de dispersión longitudinal analíticos en función del número de Péclet de la partícula $\left(P e_{p}\right)$, a diferentes porosidades para las dos condiciones de flujo que se manejan en este trabajo. Éstos se determinan, empleando la ecuación (7.1.1) y los valores de los coeficientes $A_{1}$ y $A_{2}$ de la Tabla 7.1.

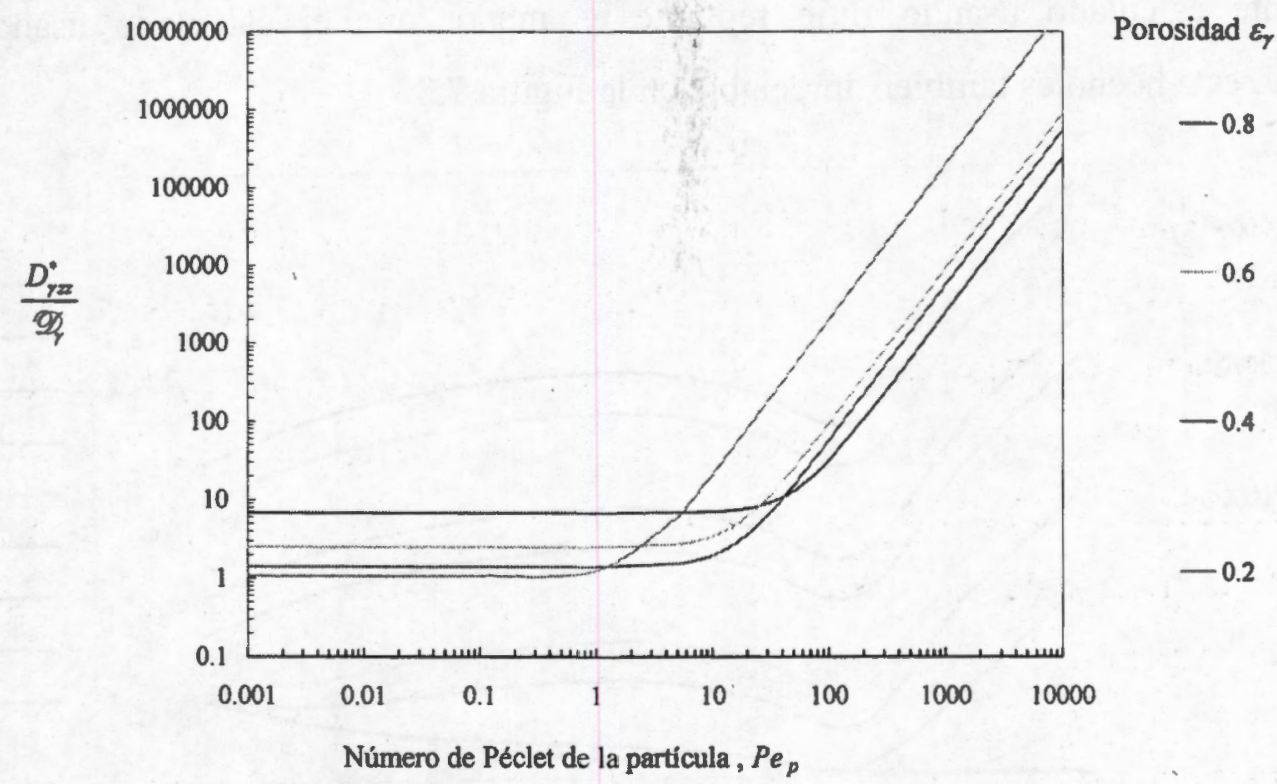

(a)

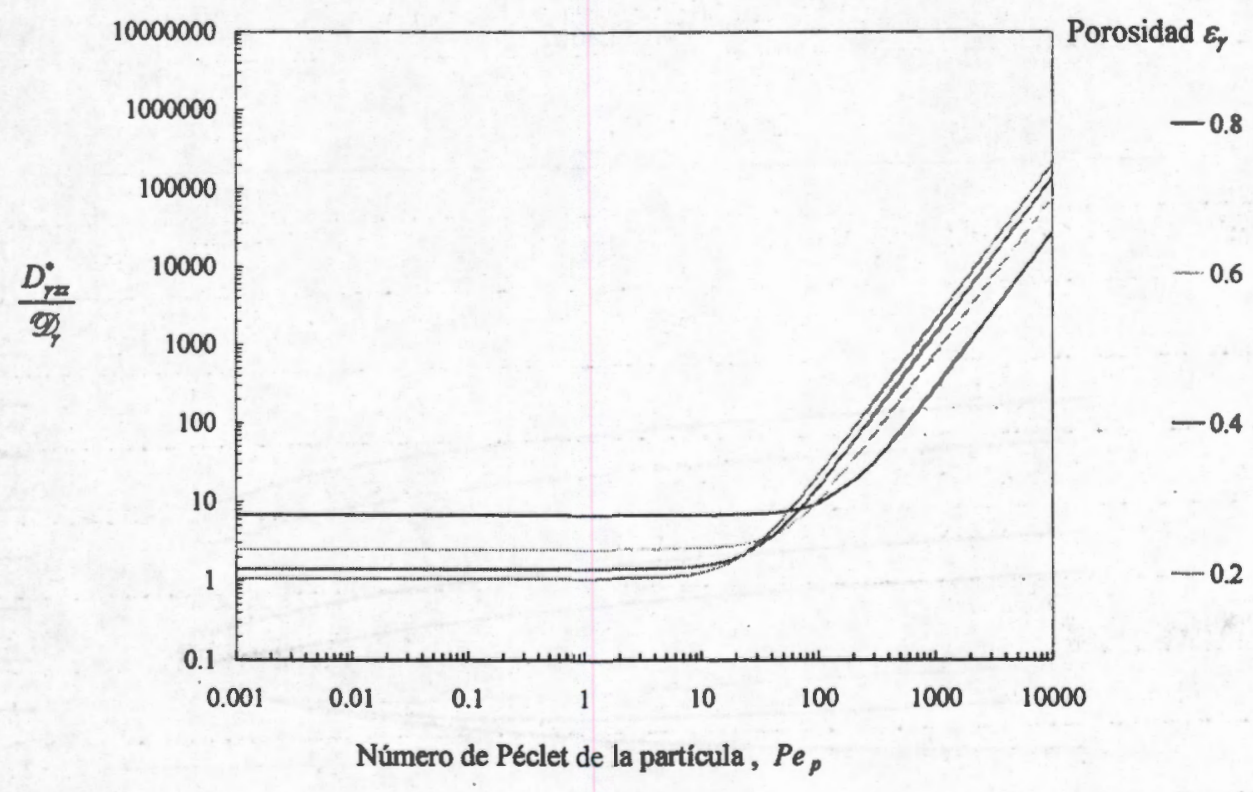

(b)

Figura 7.2 Perfil del coeficiente de dispersión longitudinal analítico para flujo potencial (a) y reptante (b)

Como se puede notar en la Figura 7.2, los perfiles del coeficiente de dispersión longitudinal tienen el mismo comportamiento para cada una de las condiciones de flujo, presentan tres 
cambios de pendiente. Para números de Péclet de partícula bajos el coeficiente presenta valores casi-constantes y menor que 10 en ambos casos, para número de Péclet alrededor de 100 el coeficiente de dispersión longitudinal tiene un crecimiento lento que después se acelera a números de Péclet grandes. Se puede observar que a una porosidad dada, el coeficiente calculado usando flujo reptante es menor que el calculado usando flujo potencial, este hecho es también apreciable en la Figura 7.3.

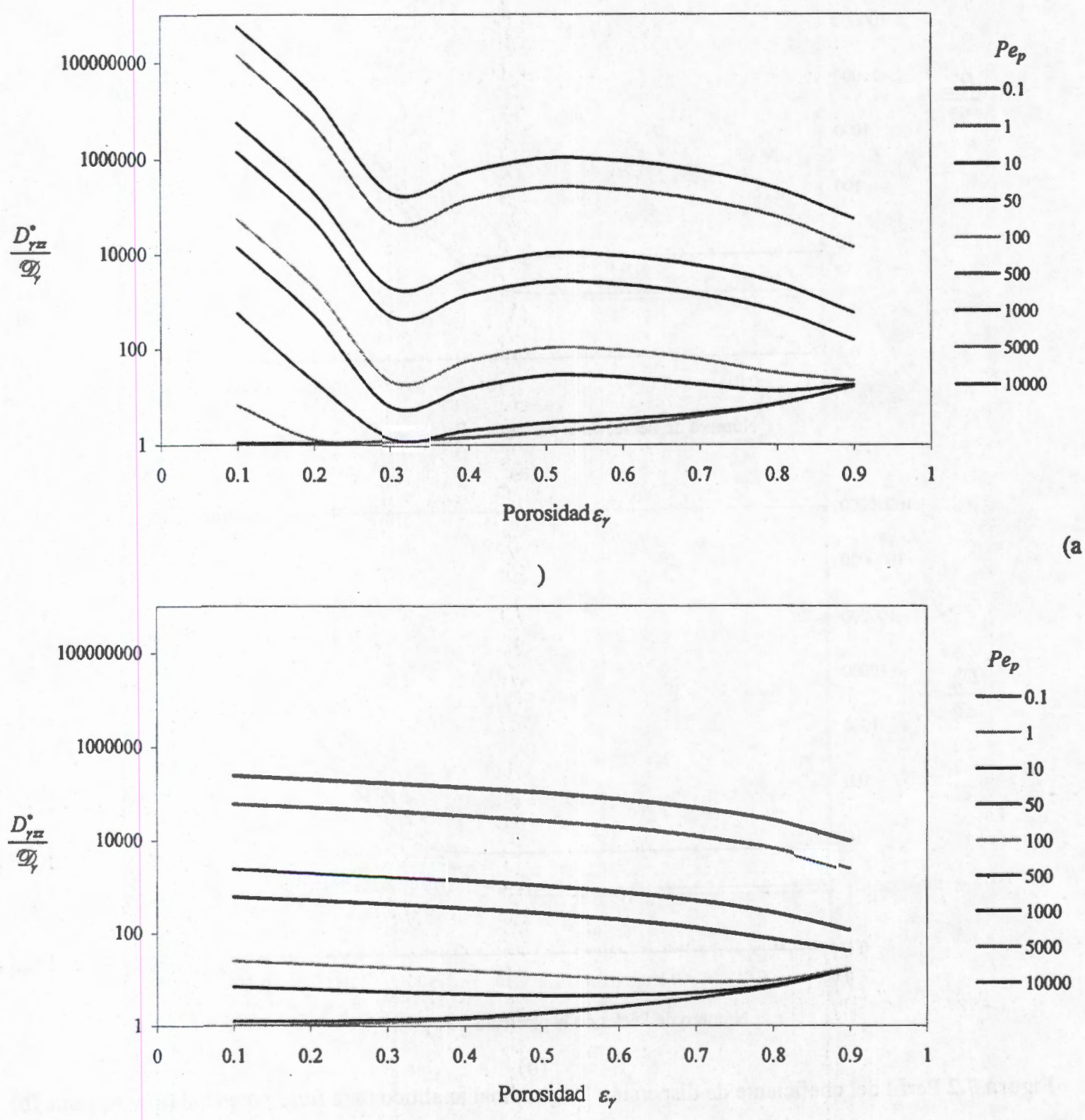

(b)

Figura 7.3 Efecto de la porosidad en el coeficiente de dispersión longitudinal para flujo potencial (a) y flujo reptante (b) 
Para poder realizar un mejor análisis de los perfiles del coeficiente longitudinal de dispersión obtenidos en este trabajo, en la Figura 7.3 se muestran algunos resultados experimentales de dicho coeficiente y se presenta un análisis de la gráfica (Bear \& Verruijt, 1987), basado en los cambios de pendiente del perfil de dispersión longitudinal la cual indica la importancia del proceso de de dispersión hidrodinámica con respecto al de difusión molecular en el proceso de dispersividad.

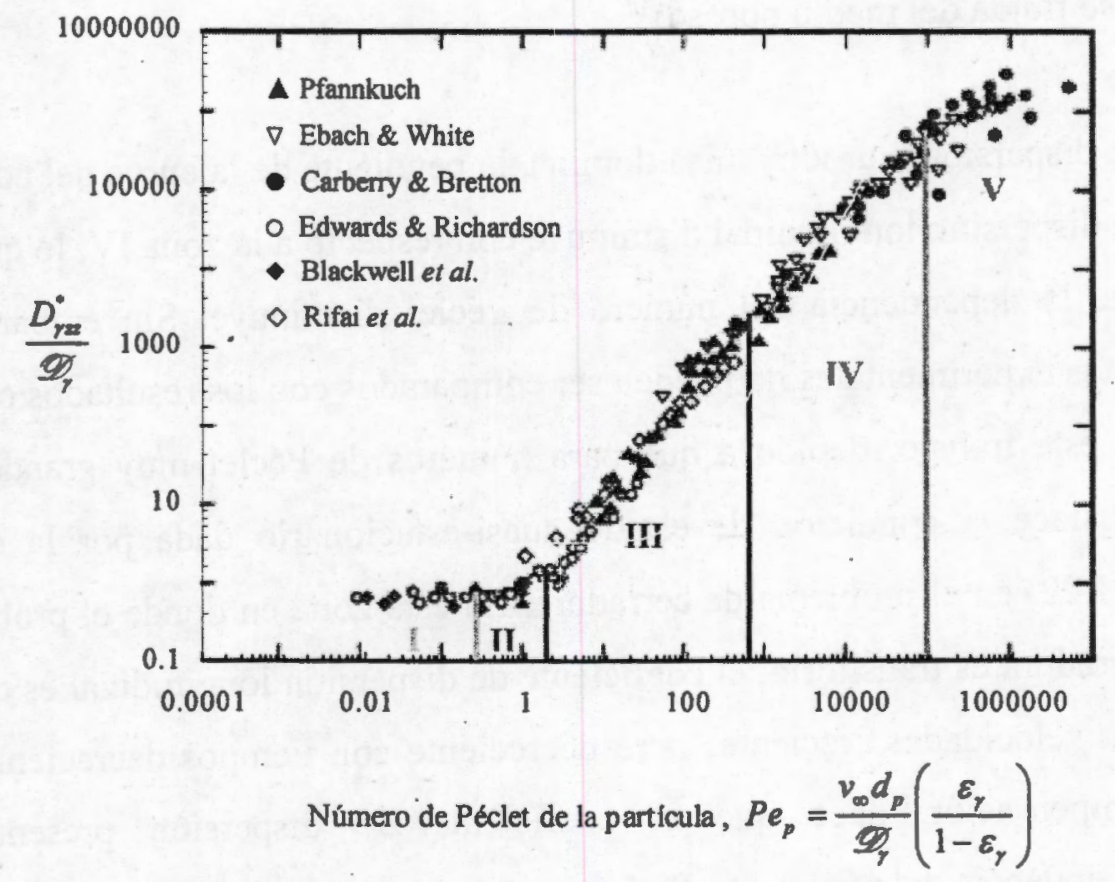

.Figura 7.4 Análisis del perfil del coeficiente longitudinal de dispersión

En la Figura 7.4 es posible identificar cinco cambios de pendiente, lo que nos lleva a reconocer cinco zonas, en las primeras cuatro zonas el coeficiente de dispersión longitudinal siempre es creciente mientras que en la última zona el coeficiente presenta un decaimiento con respecto al número de Péclet. A continuación se presenta una breve descripción de las zonas haciendo un particular énfasis en la última zona.

Zona I: Domina la difusión molecular en la propagación de la especie $A$ en la fase fluida del medio poroso 
Zona II: La difusión molecular y la dispersión hidrodinámica tienen la misma importancia

Zona III: La propagación de la especie $A$ en la fase fluida $\gamma$ se debe principalmente a la dispersión hidrodinámica

Zona IV: La dispersión hidrodinámica domina la propagación de la especie $A$ en la fase fluida del medio poroso

Zona V: La dispersión hidrodinámica domina, la pendiente de la curva del coeficiente de dispersión longitudinal disminuye con respecto a la zona IV, lo que indica que la dependencia del número de Péclet disminuye. Sin embargo estos datos experimentales no pueden ser comparados con los resultados obtenidos en este trabajo, debido a que para números de Péclet muy grandes no se satisface la condición de estado cuasi-estacionario dada por la ecuación (3.1.22) en el problema de cerradura. En está zona en donde el problema de cerradura es transitorio, el coeficiente de dispersión longitudinal es creciente con velocidades crecientes pero decreciente con tiempos decrecientes. Esta compensación hace que el coeficiente de dispersión presente poca dependencia del número de Péclet.

Han et al. (1985) realizo un estudio experimental de la dispersión axial en lechos empacados para determinar la dependencia del tiempo en el coeficiente de dispersión longitudinal experimental. Sus resultados indican que el coeficiente de dispersión longitudinal para números de Péclet grandes es constante, es decir que el coeficiente se encuentra en la zona $\mathrm{V}$, siempre que los valores del tiempo cumplan la restricción

$$
\frac{D_{y} t}{d_{p}^{2}} \geq 0.15
$$


En la Tabla 7.2 se presentan las restricciones que el tiempo debe satisfacer para que el coeficiente de dispersión longitudinal se vuelva decreciente con respecto al número de Péclet para algunos de los resultados experimentales de la Figura 7.4. En la tabla 7.2 no se presentan las restricciones de tiempo para los datos experimentales de Rifai (1956) y Edwards \& Richardson (1968) dado que estos resultados no alcanzan la zona V, es decir, la zona en donde el coeficiente longitudinal de dispersión es decreciente.

Tabla 7.2 Tiempos aproximados requeridos para alcanzar el estado cuasi-estacionario

\begin{tabular}{|l|c|}
\hline \multicolumn{1}{|c|}{ Investigador } & $\frac{\mathscr{D}_{\gamma}^{*}}{d_{p}^{2}}$ \\
\hline Pfannkuch & 0.20 \\
\hline Ebach \& White & 0.18 \\
\hline Carberry \& Bretton & 0.09 \\
\hline Han \& Carbonell & 0.15 \\
\hline
\end{tabular}

Aplicando este análisis a la Figura 7.2, podemos decir que los perfiles del coeficiente de dispersión total calculados a partir de la función de Green presentan el mismo comportamiento que los determinados experimentalmente en lechos empacados (Figura 7.3) para las zonas I, II y III.

Para el caso de flujo reptante la zona I es más importante que para flujo potencial, es decir, el primer cambio de pendiente se da para números de Péclet más bajos para flujo reptante en comparación de los observados para flujo potencial. En la zona III se observa que la pendiente del coeficiente de dispersión longitudinal es mayor para flujo potencial que para flujo reptante, esto indica que en el proceso de dispersividad, la dispersión hidrodinámica tiene mayor importancia para flujo potencial que para flujo reptante.

Los perfiles del coeficiente de dispersión longitudinal para ambos flujos que mejor se ajustan a los datos experimentales mostrados en la Figura 7.3 son los correspondientes a una porosidad de 0.25 para flujo potencial y de 0.3 para flujo reptante, tal y como se muestra en la Figura 7.4. En esta figura se presentan también los perfiles obtenidos de la solución numérica del problema de cerradura con el método $\mathrm{ADI}-2 \mathrm{D}$ para las porosidades antes mencionadas correspondientes a las condiciones de flujo. 


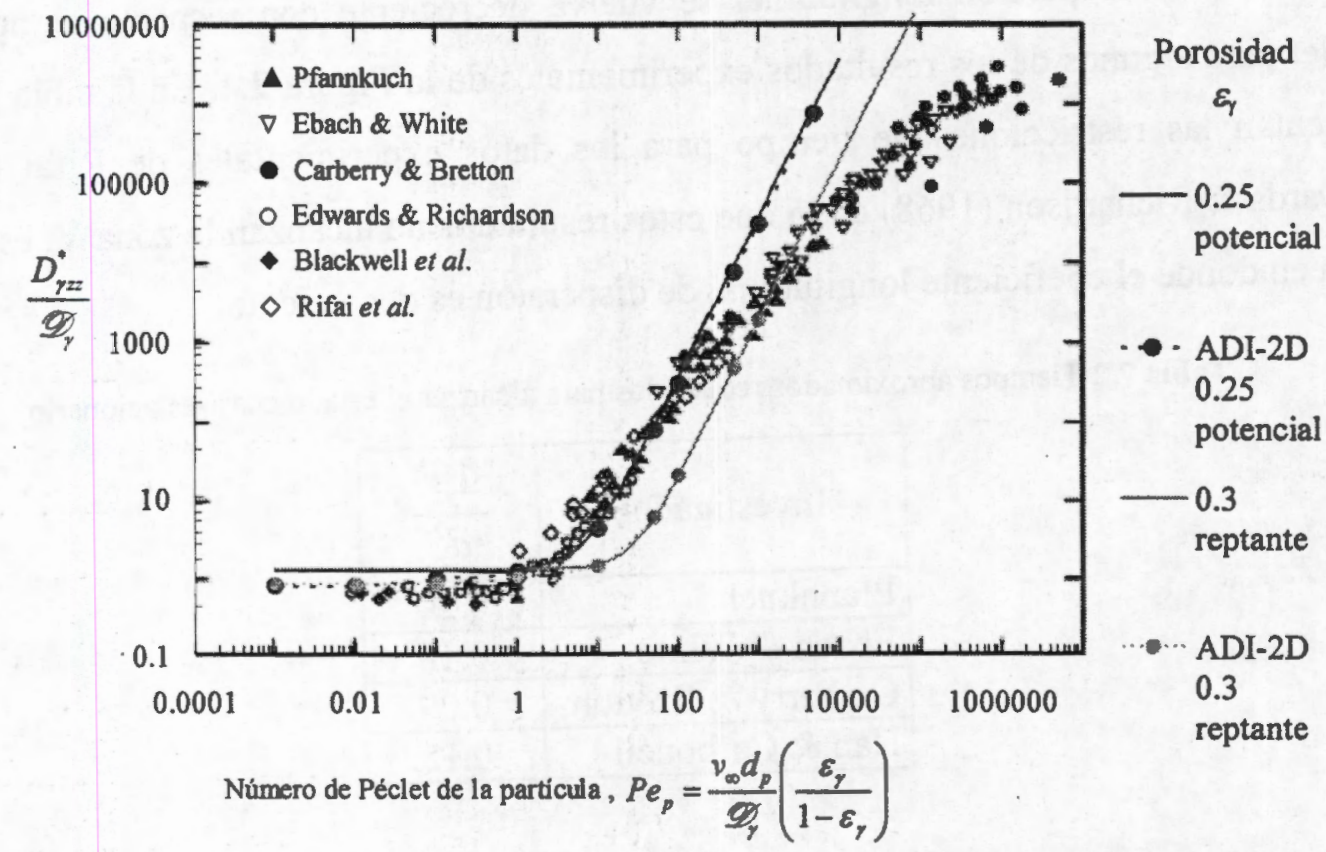

.Figura 7.4 Coeficiente de dispersión longitudinal teórico y experimental

En la Figura 7.4 se puede observar que el perfil que mejor se ajusta a los datos experimentales es el correspondiente al flujo potencial con una porosidad de 0.25 , en las zonas I, II y III. Para números de Péclet menores que 1, el perfil obtenido a partir de la función de Green se aleja de los resultados experimentales, sin embargo, el perfil resultante de la solución numérica del problema de cerradura se acerca más a los datos experimentales para este rango de números de Péclet. El perfil del coeficiente de dispersión longitudinal bajo la condición de flujo reptante a una porosidad de 0.3 presenta el mismo comportamiento. Para números de Péclet mayores que 1 , los perfiles obtenidos de la función de Green y del método ADI-2D se confunden, para ambos flujos. En la zona IV, el perfil de flujo reptante con una porosidad de 0.3 se ajusta de buena forma a los datos experimentales. En la tabla 7.3 se presentan los valores del coeficiente de dispersión longitudinal obtenidos a partir de la función de Green y del método ADI-2D para las dos condiciones de flujo que mejor se ajustan a los datos experimentales. 
Tabla 7.3: Cálculos teóricos analíticos y numéricos de los coeficientes efectivos del proceso de dispersión en la celda de Chang con geometría esférica

\begin{tabular}{|c|c|c|c|c|c|c|}
\hline \multirow{2}{*}{$P e_{p}$} & \multicolumn{2}{|c|}{ Función de Green 0.25 potencial } & \multicolumn{3}{c|}{ ADI-2D 0.25 potencial } \\
\cline { 2 - 7 } & $\begin{array}{c}\text { Difusividad } \\
\text { molecular }\end{array}$ & $\begin{array}{c}\text { Dispersión } \\
\text { Hidrodinámico }\end{array}$ & $\begin{array}{c}\text { Dispersión } \\
\text { Total }\end{array}$ & $\begin{array}{c}\text { Difusividad } \\
\text { molecular }\end{array}$ & $\begin{array}{c}\text { Dispersión } \\
\text { Hidrodinámico }\end{array}$ & $\begin{array}{c}\text { Dispersión } \\
\text { total }\end{array}$ \\
\hline 0.001 & 1.1272 & $2.691 \cdot 10^{-8}$ & 1.1272 & 0.7292 & $2.613 \cdot 10^{-8}$ & 0.7292 \\
\hline 0.01 & 1.1272 & $2.691 \cdot 10^{-6}$ & 1.1272 & 0.7307 & $2.615 \cdot 10^{-6}$ & 0.7307 \\
\hline 0.1 & 1.1272 & $2.691 \cdot 10^{-4}$ & 1.1275 & 0.8990 & $2.677 \cdot 10^{-4}$ & 0.8993 \\
\hline 1 & 1.1272 & $2.691 \cdot 10^{-2}$ & 1.1541 & 1.0496 & $2.689 \cdot 10^{-2}$ & 1.0765 \\
\hline 10 & 1.1272 & 2.6906 & 3.8177 & 1.0991 & 2.6903 & 3.7893 \\
\hline 50 & 1.1272 & 67.2639 & 68.3911 & 1.1147 & 67.2611 & 68.3758 \\
\hline 100 & 1.1272 & 269.0557 & 270.1829 & 1.1138 & 269.0444 & 270.1582 \\
\hline 500 & 1.1272 & 6726.3926 & 6727.5198 & 1.1115 & 6726.2725 & 6727.3840 \\
\hline
\end{tabular}

\begin{tabular}{|c|c|c|c|c|c|c|}
\hline \multirow{2}{*}{$P_{e_{p}}$} & \multicolumn{3}{|c|}{ Función de Green 0.3 reptante } & \multicolumn{3}{c|}{ ADI-2D 0.3 reptante } \\
\cline { 2 - 7 } & $\begin{array}{c}\text { Difusividad } \\
\text { molecular }\end{array}$ & $\begin{array}{c}\text { Dispersión } \\
\text { Hidrodinámico }\end{array}$ & $\begin{array}{c}\text { Dispersión } \\
\text { Total }\end{array}$ & $\begin{array}{c}\text { Difusividad } \\
\text { molecular }\end{array}$ & $\begin{array}{c}\text { Dispersión } \\
\text { Hidrodinámico }\end{array}$ & $\begin{array}{c}\text { Dispersión } \\
\text { total }\end{array}$ \\
\hline 0.001 & 1.1905 & $1.7076 \mathrm{E}-09$ & 1.1905 & 0.7415 & $1.798 \mathrm{E}-09$ & 0.7415 \\
\hline 0.01 & 1.1905 & $1.7076 \mathrm{E}-07$ & 1.1905 & 0.7422 & $1.796 \mathrm{E}-07$ & 0.7422 \\
\hline 0.1 & 1.1905 & $1.7076 \mathrm{E}-05$ & 1.1905 & 0.7491 & $1.779 \mathrm{E}-05$ & 0.7492 \\
\hline 1 & 1.1905 & $1.7076 \mathrm{E}-03$ & 1.1922 & 0.9242 & $1.684 \mathrm{E}-03$ & 0.9259 \\
\hline 10 & 1.1905 & 0.1708 & 1.3612 & 1.1120 & 0.1703 & 1.2823 \\
\hline 50 & 1.1905 & 4.2691 & 5.4595 & 1.1701 & 4.2684 & 5.4385 \\
\hline 100 & 1.1905 & 17.0763 & 18.2668 & 1.1773 & 17.0753 & 18.2526 \\
\hline 500 & 1.1905 & 426.9070 & 428.0975 & 1.1823 & 426.8851 & 428.0674 \\
\hline
\end{tabular}

Los resultados de Gunn y Pryce (1969) del coeficiente de dispersión longitudinal mostrados en la Figura 7.5 son para arreglos cúbico de esferas y empaques aleatorios de esferas. Estos datos a pesar de no ser calculados en medios poros reales resultan ser muy atractivos debido a que reflejan la importancia del orden y el desorden en los procesos de dispersión. Los datos correspondientes a los arreglos cúbicos presentan mayor dependencia al número de Péclet que aquellos calculados en empaques aleatorios de esferas. Para números de Péclet en el rango de 1-100 los resultados de Gunn y Pryce están relativamente cercanos, mientras que para números de Péclet mayores que 100 los el coeficiente de dispersión 
longitudinal da valores más grandes para los lechos empacados aleatorios. En la Figura 7.5 se muestran también los perfiles del coeficiente de dispersión longitudinal obtenidos analíticamente, es decir a partir de la función de Green, en la celda de Chang con geometría esférica para las condiciones de flujo reptante y potencial que más se acercan a los datos reportados por Gunn y Pryce. Se puede observar que los datos para flujo reptante están relativamente lejos de los datos de Gunn y Pryce en el rango de números de Péclet de 11000. El perfil del coeficiente dispersión longitudinal para flujo potencial con una porosidad de 0.25 ajusta muy bien los datos de Gunn y Pryce para arreglos cúbicos de esferas.

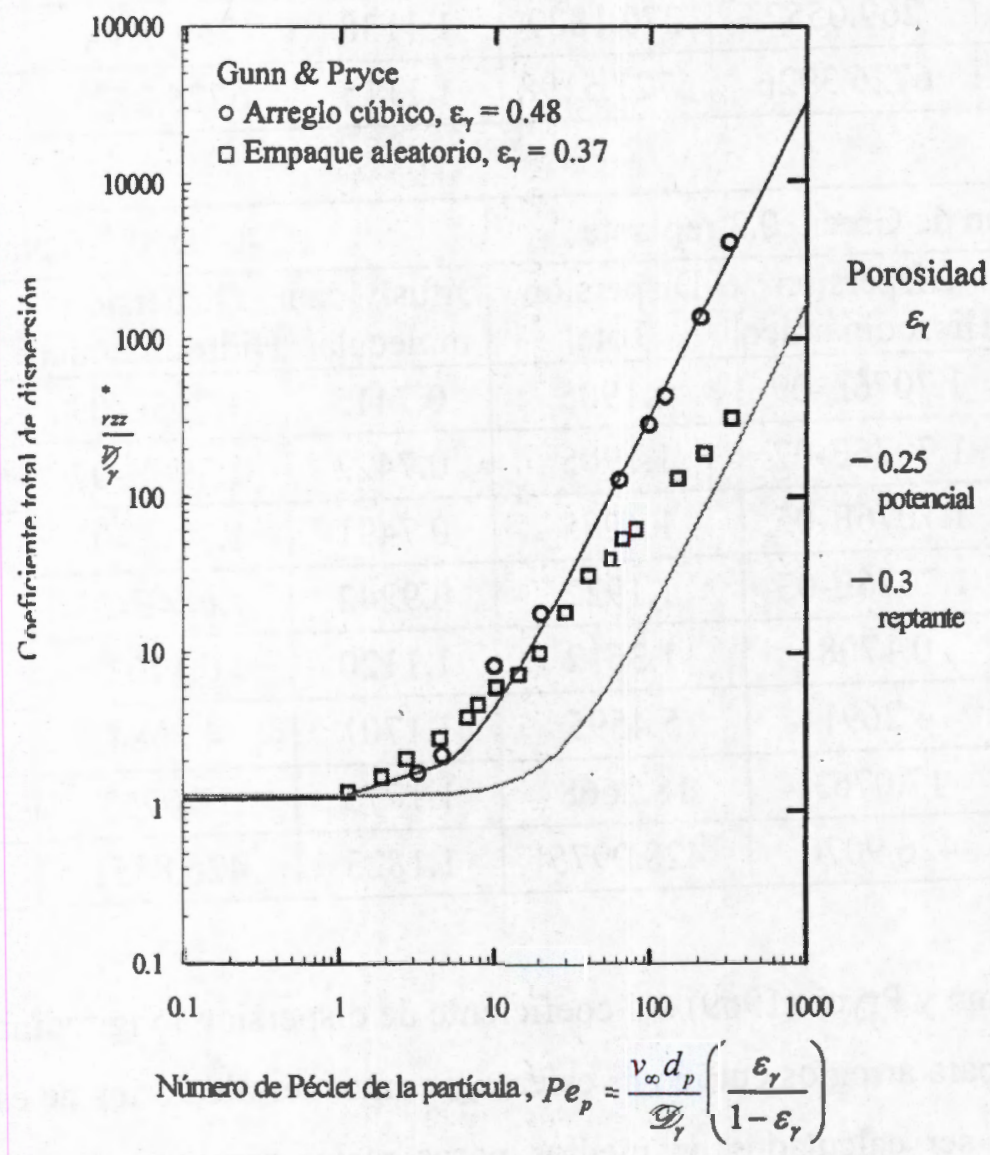

Figura 7.5 Coeficiente de dispersión longitudinal experimental en celdas ordenadas y desordenadas y teórico en la celda de Chang con geometría esférica

El problema de cerradura dado por las ecuaciones (3.1.25)-(3.1.27) ha sido resuelto a través de diferentes métodos numéricos por distintos investigadores que trabajan de manera 
independiente. Eidsath et al. (1983) empleo el elemento finito como técnica computacional para resolver el problema de cerradura en diversas celdas unitarias bidimensionales de arreglos cuadrados y cilíndricos, el elemento finito también fue usado por Edwards et al. (1991, 1993), Sahraoui y Kaviany (1994) usaron las diferencias finitas en celdas bidimensionales en donde las particulares se modelaron por cilindros cuadrados y circulares, Quintard y Whitaker (1994) resolvieron el problema de cerradura en celdas bidimensionales de arreglos de cilindros usando la técnica de volumen finito. En la Figura 7.6 se comparan los resultados de los investigadores antes mencionados con los valores del coeficiente de dispersión longitudinal en la celda de Chang con geometría esférica para flujo potencial, calculados en este trabajo analíticamente a través de la función de Green y numéricamente con el método ADI-2D. Reportando únicamente el perfil que mejor se ajusta a los datos antes mencionados, el cual está asociado a una porosidad de 0.7. Cabe mencionar que ninguno de los perfiles del coeficiente de dispersión longitudinal para flujo reptante se ajustó a los datos de los investigadores.

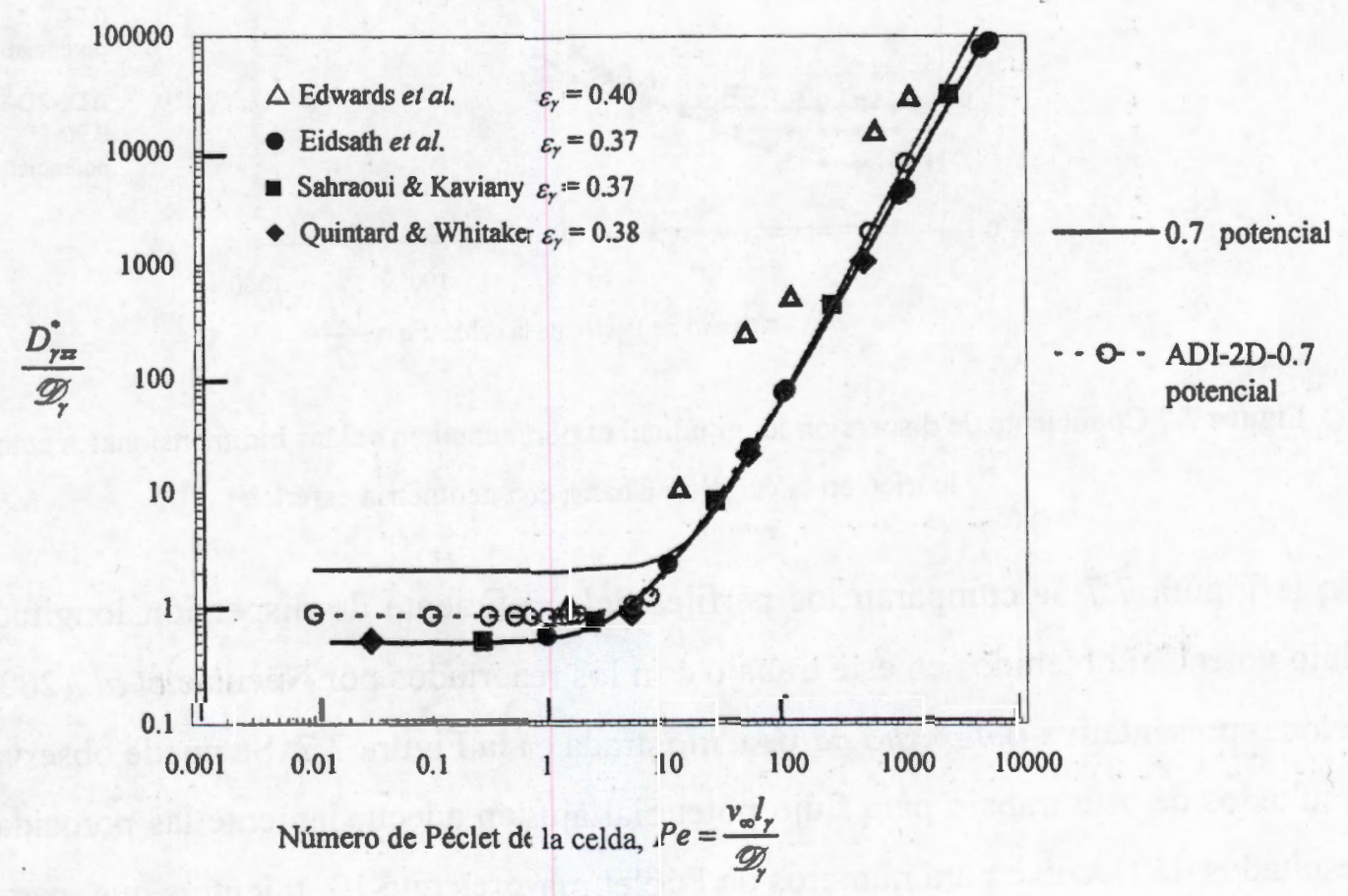

Figura 7.6 Coeficiente de dispersión longitudinal experimental en celdas bidimensionales de arreglos de cuadrados y cilindros y teórico en la celda de Chang con geometría esférica 
En la Figura 7.6 se puede observar que para números de Péclet menores que 10, los valores del coeficiente de dispersión longitudinal calculados en este trabajo están muy alejados de los datos de los investigadores, para un rango de números de Péclet de 10-100 los datos obtenidos en este trabajo se ajustan de buena manera a los datos de los investigadores, mientras que para números de Péclet mayores que 100 estos se alejan de nueva cuenta de los resultados previos. Estas discrepancias pueden atribuirse a las diferencias en las microestructuras y a que los resultados previos fueron calculados para celdas bidimensionales mientras que los obtenidos en este trabajo son calculados en una celda tridimensional.

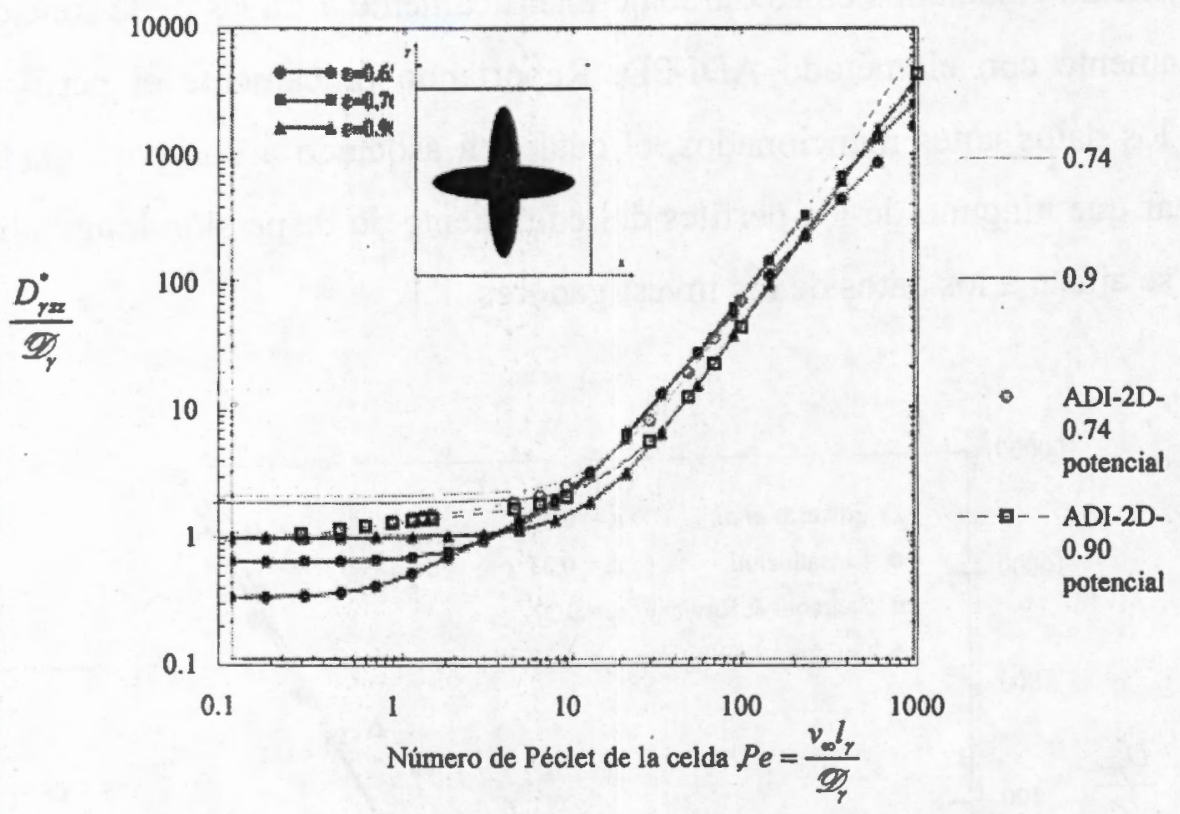

Figura 7.7 Coeficiente de dispersión longitudinal experimental en celdas bidimensionales complejas y teórico en la celda de Chang con geometría esférica

En la Figura 7.7 se comparan los perfiles del coeficiente de dispersión longitudinal para flujo potencial obtenidos en este trabajo con los reportados por Neculae et al. (2002) para la celda representativa del medio poroso, mostrada en la Figura 7.7. Se puede observar que los resultados de este trabajo para flujo potencial ajustan adecuadamente las porosidades y los resultados de Neculae para números de Péclet mayores que 10, mientras que, para números de Péclet menores que 10, los resultados están lejos de coincidir. 


\subsection{Coeficiente transversal de dispersión total}

Equation Chapter 7 Section 2

El coeficiente de dispersión transversal en la celda de Chang con geometría esférica corresponde a la componente $x x$ del tensor de dispersión total. De la solución al problema de cerradura usando la función de Green, se tiene que la expresión de este coeficiente puede expresarse como sigue

$$
\frac{D_{r x x}^{*}}{\mathscr{D}_{r}}=1+a_{1}+a_{2} P e^{2}
$$

Donde

$$
\begin{aligned}
& a_{2}=-\frac{1}{V_{\gamma}} \int_{0}^{2 \pi} \int_{0}^{\pi} \int_{\xi_{p}}^{\xi_{\xi}} \tilde{U}_{\gamma X} B_{X}^{\text {convecivo }}(\xi, \theta, \phi) \xi^{2} \operatorname{sen} \theta d \xi d \theta d \phi \\
& a_{1}=-\frac{1}{V_{\gamma}} \int_{0}^{2 \pi} \int_{0}^{\pi} \mathbf{k} \cdot e_{\xi} B_{X}^{\text {dijusivo }}\left(\xi_{p}, \theta, \phi\right) \operatorname{sen} \theta d \theta d \phi
\end{aligned}
$$

Para el cálculo de los coeficientes $a_{1}$ y $a_{2}$ dados por la ecuación (7.2.2) se programó la función de Green que es una serie infinita dependiente de las tres coordenadas esféricas dada por la ecuación (4.6.5), las integrales triples y dobles involucradas en la determinación de estos coeficientes se realizaron numéricamente basándose en el algoritmo de Simpson para integrales dobles combinado con el de Simpson compuesto. En la tabla 7.4 se presenta el valor de los coeficientes $a_{1}$ y $a_{2}$ calculados usando la función de Green para el número de Péclet de la partícula $\left(P e_{p}\right)$ a diferentes porosidades y para flujo reptante y potencial. Es importante mencionar que de manera análoga al coeficiente de dispersión longitudinal el coeficiente que acompaña al término $P e_{p}$ en el polinomio de segundo grado que determina al coeficiente de dispersión transversal es nulo. 
Tabla 7.4 Coeficientes del coeficiente de dispersión transversal para el número de Péclet de la partícula $\left(P e_{p}\right)$ para flujo potencial y reptante

\begin{tabular}{|c|c|c|}
\hline $\begin{array}{c}\text { Porosidad y condición } \\
\text { de flujo }\end{array}$ & $a_{2}$ & $a_{1}$ \\
\hline 0.22 potencial & $2.839 \mathrm{E}-02$ & 1.0000 \\
\hline 0.29 potencial & $1.899 \mathrm{E}-03$ & 1.0000 \\
\hline 0.7 reptante & $4.386 \mathrm{E}-05$ & 0.9804 \\
\hline 0.3 reptante & $1.953 \mathrm{E}-04$ & 0.9987 \\
\hline
\end{tabular}

En este trabajo, los valores del coeficiente de dispersión transversal son menores a los del coeficiente de dispersión longitudinal, lo que es consiste con los resultados previos de dispersión. En la Figura 7.8 se muestras datos del coeficiente de dispersión transversal obtenidos experimentalmente en lechos empacados junto con los resultados de este trabajo para las condiciones de flujo potencial y reptante y a las porosidades que mejor se ajustan a los datos previos.

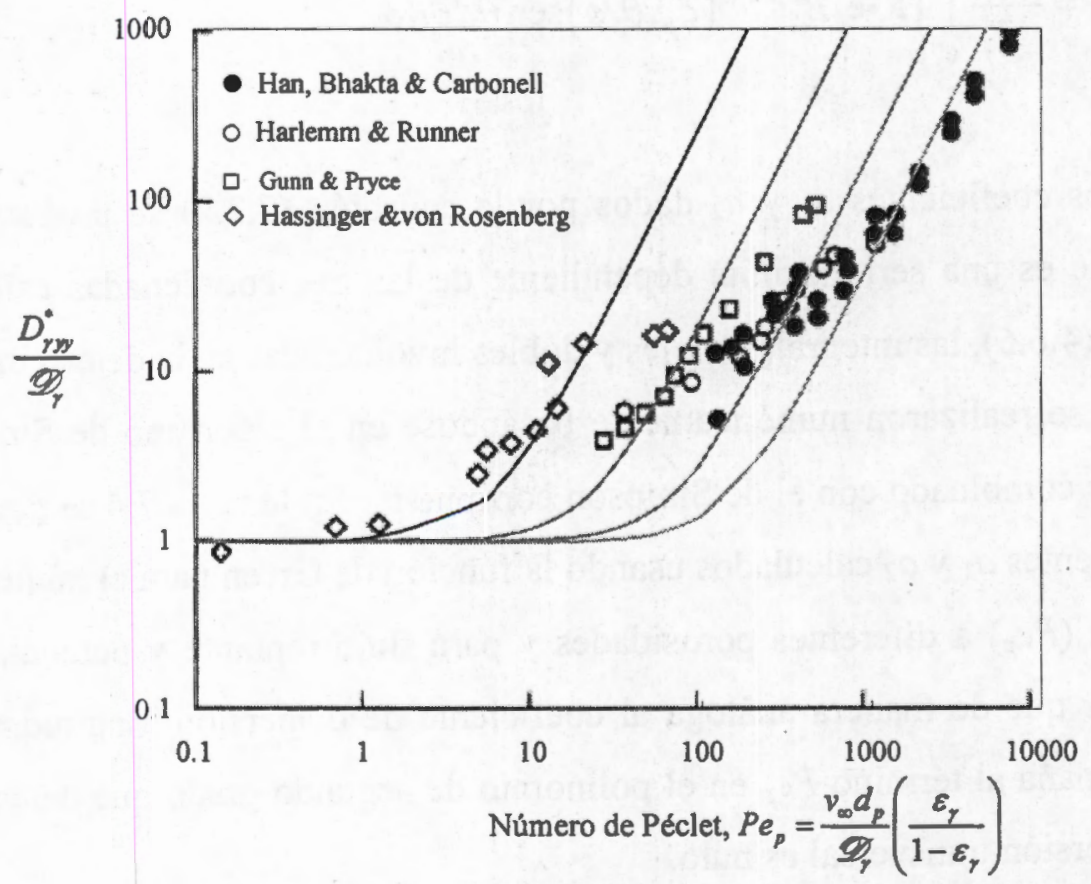

Porosidad

$\varepsilon_{\gamma}$

-0.3 reptante

0.7 reptante

-0.22 potencial

0.29 potencial

Figura 7.8: Coeficiente de dispersión transversal teórico y experimental 
Como se puede observar en la Figura 7.8, los datos experimentales de Hassinger \& von Rosenberg son ajustados por los valores calculados del coeficiente de dispersión transversal en la celda de Chang con geometría esférica por aquellos referentes a una porosidad de 0.22 y flujo potencial, para una rango aproximado de números de Péclet de 0.1 a 30. Los datos de Gunn \& Pryce son ajustados correctamente en un intervalo de números de Péclet de10 a 100 por los coeficientes transversales asociados a una porosidad de 0.29 y flujo potencial, para números de Péclet mayores que 100, los valores calculados en este trabajo se alejan de los reportados por estos investigadores. Los datos experimentales Han, Bhakta y Carbonell muestran un comportamiento diferente a los obtenidos en este trabajo, sin embargo estos muestran semejanza en el orden de magnitud.

Los resultados reportados en la literatura para el coeficiente de dispersión transversal están lejos de acercarse a los datos experimentales mostrados en la Figura 7.8 debido a su poca dependencia en el número de Péclet. No siendo este el caso para los calculados en este trabajo con la función de Green, de la ecuación (7.2.1) se puede observar que los resultados de este trabajo para el coeficiente de dispersión transversal presentan dependencia del cuadro del número de Péclet. Los coeficientes de la ecuación (7.2.1) y definidos por las expresiones (7.2.2) permiten de alguna manera filtrar las complejidades asociadas con el medio poroso. El hecho de no poder haber obtenido un solo perfil del coeficiente de dispersión longitudinal para cada uno de los flujos que pueda modelar los datos experimentales de la Figura 7.7 puede deberse a que en medios porosos reales las heterogeneidades generan más movimiento lateral. 



\section{Conclusiones}

En este trabajo, se encontró la solución analítica del problema de cerradura que permite calcular los coeficientes efectivos de transporte del proceso de dispersión pasiva en un medio poroso homogéneo usando las funciones de Green. Esta solución es válida para cualquier región elemental simétrica en el espacio que pueda describir la fase fluida del me dio poroso homogéneo. Esta solución se aplico a la celda de Chang con geometría esférica que es la celda unitaria que modela un medio poroso homogéneo espacialmente periódico, en la que fue posible determinar la expresión analítica de la función de Green usando expansiones en series de Fourier en los esféricos armónicos. Por otro lado, se resolvió numéricamente el problema de cerradura usando el método $\mathrm{ADI}$ de diferencias finitas. $\mathrm{Al}$ comparar los resultados obtenidos, se observo que para números de Péclet menores que uno, los valores del coeficiente de dispersión longitudinal numérico son menores que los obtenidos de la solución analítica del problema de cerradura. Esta diferencia en los valores recae sobre el coeficiente de difusión molecular (el coeficiente de dispersión hidrodinámica numérico y analítico tiende a cero para números de Péclet menores que uno). Para números de Péclet mayores que uno los valores del coeficiente de dispersión longitudinal analíticos y numéricos son prácticamente idénticos. Se determino que los resultados de este trabajo para flujo potencial para números de Péclet menores que 10000 son los que en general se ajustan de mejor forma a los datos experimentales y teóricos reportados en la literatura. De la comparación de estos resultados se observo que el método ADI proporciona valores del coeficiente de dispersión longitudinal más apegados a la realidad para números de Péclet menores que 1. De esta manera se puede concluir que la solución numérica y analítica del problema de cerradura en conjunto permiten tener valores correctos del coeficiente de dispersión longitudinal para números de Péclet menores que 1000. 



\section{Nomenclatura}

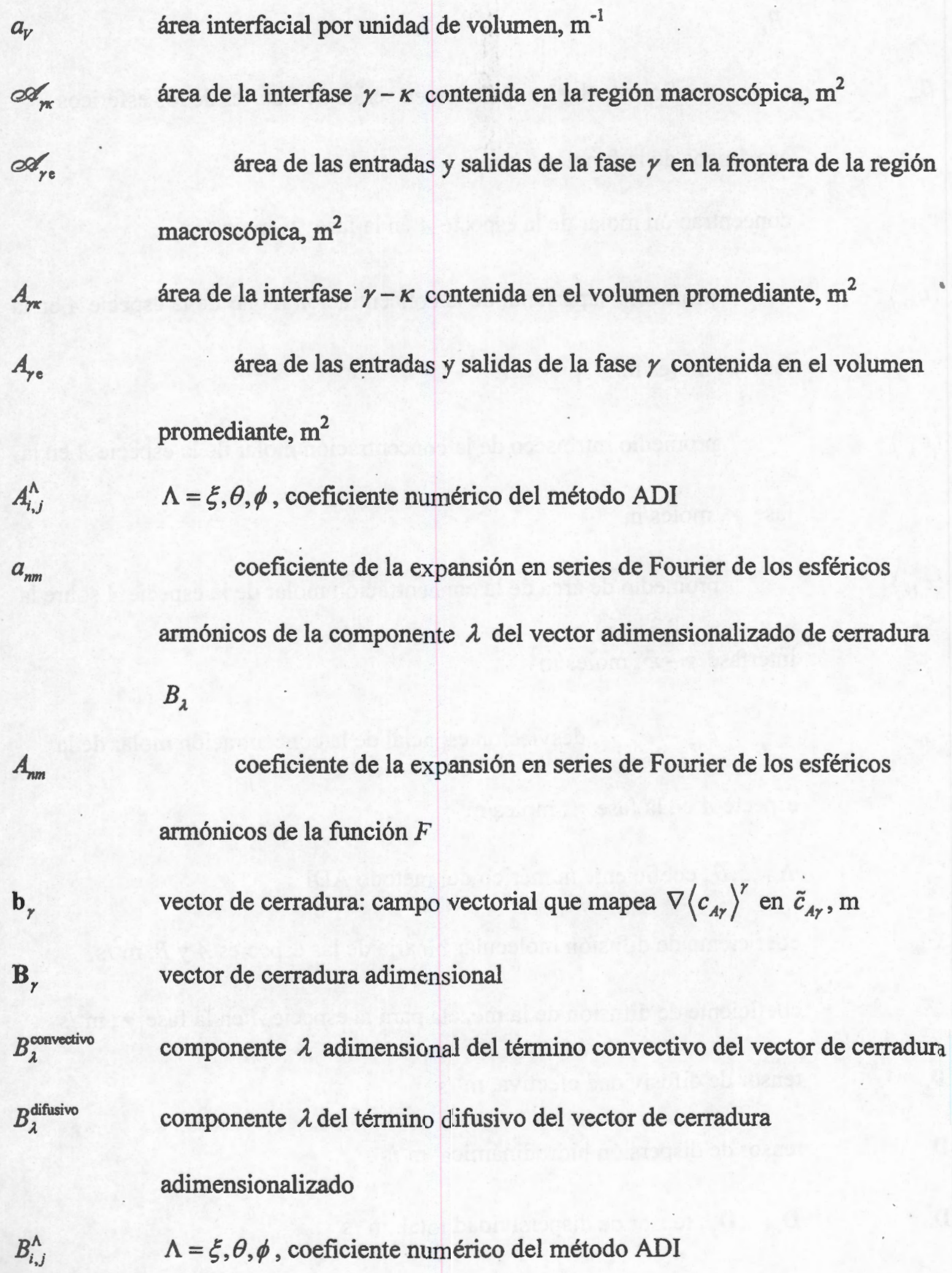


$b_{n m}$

$B_{n m}$

$c_{A y}$

$\left\langle c_{A \gamma}\right\rangle$

$\left\langle c_{A \gamma}\right\rangle^{\gamma}$

$\left\langle c_{A r}\right\rangle_{p}$

$\tilde{c}_{A \gamma}$

$C_{i, j}^{\Lambda}$

$\mathscr{P}_{A B}$

$\mathscr{D}$

$\mathbf{D}_{\text {eff }}$

$\mathbf{D}_{\gamma}$

$\mathbf{D}_{\gamma}^{*}$ coeficiente de la expansión en series de Fourier de los esféricos

armónicos de la componente $\lambda$ del vector adimensionalizado de cerradura $B_{\lambda}$

coeficiente de la expansión en series de Fourier de los esféricos armónicos de la función $F$ concentración molar de la especie $A$ en la fase $\gamma$, moles $/ \mathrm{m}^{3}$

promedio superficial de la concentración molar de la especie $A$ en la fase $\gamma$, moles $/ \mathrm{m}^{3}$

promedio intrínseco de la concentración molar de la especie $A$ en la fase $\gamma$, moles $/ \mathrm{m}^{3}$

promedio de área de la concentración molar de la especie $A$ sobre la interfase $\gamma-\kappa$, moles $/ \mathrm{m}^{3}$

$$
c_{A \gamma}-\left\langle c_{A \gamma}\right\rangle^{\gamma} \text {, desviación espacial de la concentración molar de la }
$$

especie $A$ en la fase $\gamma$, moles $/ \mathrm{m}^{3}$

$\Lambda=\xi, \theta$, coeficiente numérico del método $\mathrm{ADI}$

coeficiente de difusión molecular binaria de las especies $A$ y $B, \mathrm{~m}^{2} / \mathrm{s}$

coeficiente de difusión de la mezcla para la especie $A$ en la fase $\gamma, \mathrm{m}^{2} / \mathrm{s}$

tensor de difusividad efectiva, $\mathrm{m}^{2} / \mathrm{s}$

tensor de dispersión hidrodinámica, $\mathrm{m}^{2} / \mathrm{s}$

$\mathbf{D}_{e f f}+\mathbf{D}_{\gamma}$, tensor de dispersividad total, $\mathrm{m}^{2} / \mathrm{s}$ 


\begin{tabular}{|c|c|}
\hline$d_{p}$ & $2 r_{o}$, diámetro efectivo de la partícula, $\mathrm{m}$ \\
\hline$D_{\Lambda i, j, k}^{p}$ & $\Lambda=\xi, \theta, \phi$, coeficiente numérico del método $\mathrm{ADI}$ al el instante $p$ \\
\hline $\mathbf{e}_{\gamma}$ & vector ortonormal de la dirección radial de las coordenadas esféricas \\
\hline $\mathbf{e}_{\xi}$ & vector ortonormal de la dirección radial de las coordenadas esféricas \\
\hline e. & vector ortonormal de la dirección $\theta$ de las coordenadas esféricas \\
\hline $\mathbf{e}_{\theta}$ & vector ortonormal de la dirección $\phi$ de las coordenadas esféricas \\
\hline$F$ & $F: V_{\gamma} \subset \mathbb{R}^{3} \rightarrow \mathbb{R}$, término no homogéneo de la ecuación de Poisson, \\
\hline$f$ & $F: A_{m} \subset \mathbb{R}^{2} \rightarrow \mathbb{R}$, término no homogéneo de la condición de frontera de \\
\hline & Neumann en la interfase en la ecuación de Poisson \\
\hline g & aceleración gravitacional, $\mathrm{m}^{2} / \mathrm{s}$ \\
\hline G & función de Green del problema de cerradura en $V_{\gamma}$ \\
\hline$G^{*}$ & función de Green de la ecuación de Poisson en $V_{r}$ \\
\hline$G_{n}$ & función de Green para del problema de valores a la frontera del \\
\hline & término radial de la ecuación de Poisson en $V_{r}$ \\
\hline i & vector ortonormal de la dirección $x$ de las coordenadas cartesianas \\
\hline $\mathbf{j}$ & vector ortonormal de la dirección $y$ de las coordenadas cartesianas \\
\hline k & vector ortonormal de la dirección $z$ de las coordenadas cartesianas \\
\hline$K_{n}$ & núcleo o función generadora de los esféricos armónicos \\
\hline$l_{\gamma}$ & longitud característica de la fase $\gamma, \mathrm{m}$ \\
\hline$l_{i}$ & $i=1,2,3$, componentes del vectores de lattice, $\mathrm{m}$ \\
\hline
\end{tabular}



longitud característica, $\mathrm{m}$ operador diferencial auto adjunto de dispersión operador diferencial no-auto adjunto de dispersión longitud característica para $\nabla \varepsilon_{y}, \mathrm{~m}$ longitud característica para $\nabla\left\langle c_{A \gamma}\right\rangle^{\gamma}, \mathrm{m}$ longitud característica para $\nabla \nabla\left\langle c_{A \gamma}\right\rangle^{\gamma}, \mathrm{m}$ operador diferencial de Laplace número de nodos del intervalo disctertizado de la coordenada $\xi$ número de nodos del intervalo disctertizado de la coordenada $\theta$

n vector normal unitario dirigido de la fase $\kappa$ a la fase $\gamma$

$n_{\lambda}$ componente $\lambda$ del vector normal unitario $\mathbf{n}$ vector normal unitario dirigido de la fase $\gamma$ a la fase $\kappa$

$n_{c}$ número de especies en la fase fluida presión modificada adimensionalizada

$P_{0}$ presión característica modificada, $\mathrm{N}$ número de Péclet, $P e=\frac{v_{\infty} r_{0}}{\mathscr{D}_{\varphi}}$ número de Péclet de la partícula, $P e_{p}=\frac{\nu_{\infty} d_{p}}{\mathscr{D}_{\gamma}}\left(\frac{\varepsilon_{\gamma}}{1-\varepsilon_{\gamma}}\right)$ polinomio de Legendre de grado $\mathrm{n}$ $P_{n}^{m}$ función asociada de Legendre 


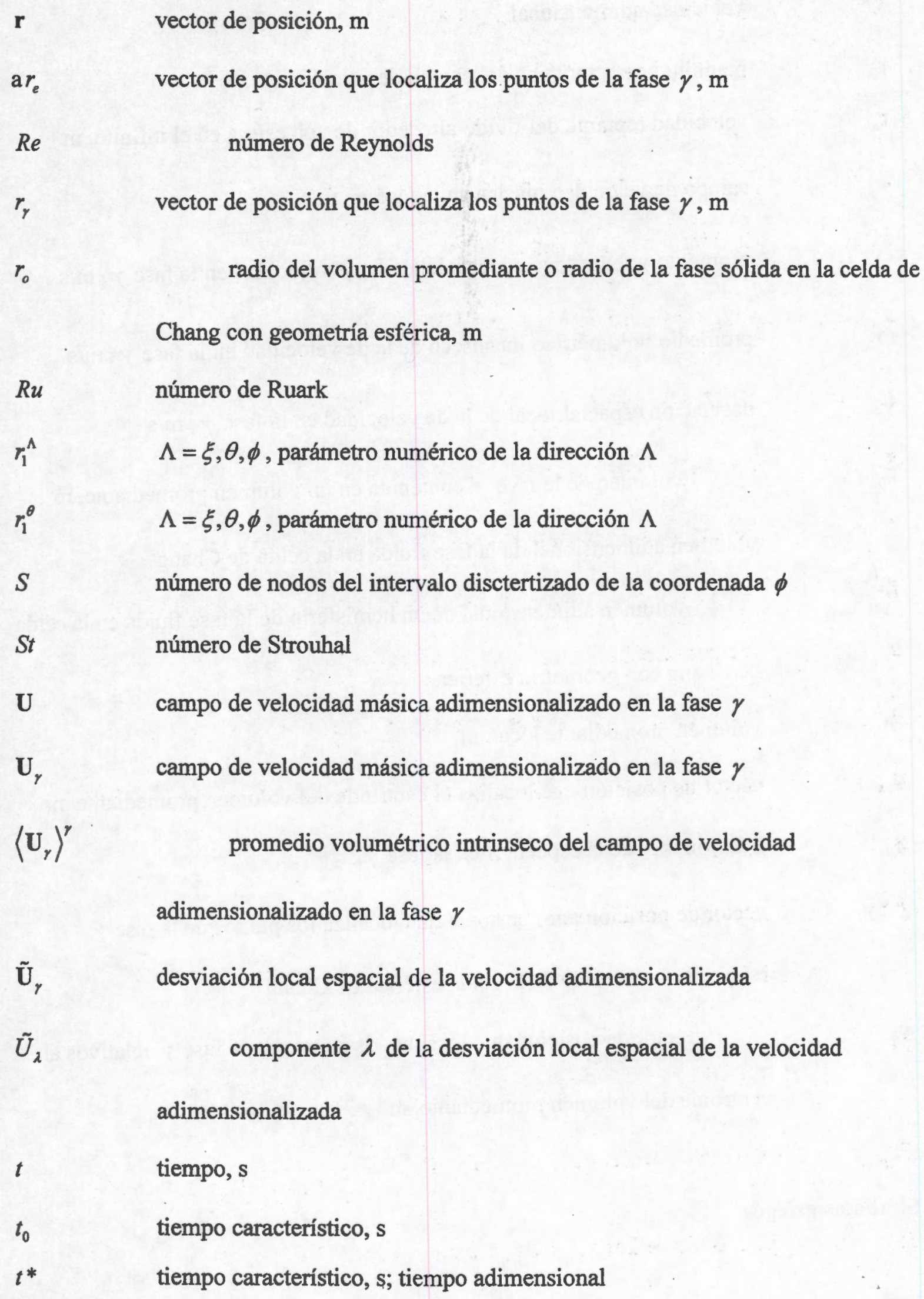




\begin{tabular}{|c|c|}
\hline $\mathbf{v}^{*}$ & velocidad adimensional \\
\hline$v_{0}$ & magnitud característica del campo de velocidad \\
\hline$v_{\infty}$ & velocidad reptante del fluido alrededor de una esfera en el infinito, $m$ \\
\hline $\mathbf{v}_{\gamma}$ & campo de velocidad másica en la fase $\gamma, \mathrm{m} / \mathrm{s}$ \\
\hline$\left\langle\mathbf{v}_{r}\right\rangle$ & promedio volumétrico superficial de la de velocidad en la fase $\gamma, \mathrm{m} / \mathrm{s}$ \\
\hline$\left\langle\mathbf{v}_{r}\right\rangle^{y}$ & promedio volumétrico intrínseco de la de velocidad en la fase $\gamma, \mathrm{m} / \mathrm{s}$ \\
\hline$\tilde{\mathbf{v}}_{\gamma}$ & desviación espacial local de la de velocidad en la fase $\gamma, \mathrm{m} / \mathrm{s}$ \\
\hline$V_{\gamma}$ & volumen de la fase $\gamma$ contenida en un volumen promediante, $\mathrm{m}^{3}$ \\
\hline$V_{r}^{*}$ & $\begin{array}{l}\text { volumen adimensional de la fase fluida en la celda de Chang } \\
\text { volumen adimensional de un hemisferio de la fase fluida en la celda }\end{array}$ \\
\hline & de Chang con geometría esférica \\
\hline $\mathscr{V}$ & volumen promediante local, $\mathrm{m}^{3}$ \\
\hline $\mathbf{x}$ & vector de posición que localiza el centroide del volumen promediante, $\mathrm{m}$ \\
\hline$x_{A y}$ & fracción mol de la especia $A$ en la fase $\gamma$ \\
\hline $\mathbf{y}, \mathbf{y}_{0}, \mathbf{y}_{1}$ & $\begin{array}{l}\text { vector de posición adimensional que localiza los puntos de la fase } \gamma \\
\text { relativos al centroide de la celda esférica de Chang }\end{array}$ \\
\hline $\mathbf{y}_{\gamma}$ & $\begin{array}{l}\text { vector de posición que localiza los puntos de la fase } \gamma \text { relativos al } \\
\text { centroide del volumen promediante, } \mathrm{m}\end{array}$ \\
\hline
\end{tabular}

\section{Símbolos griegos}




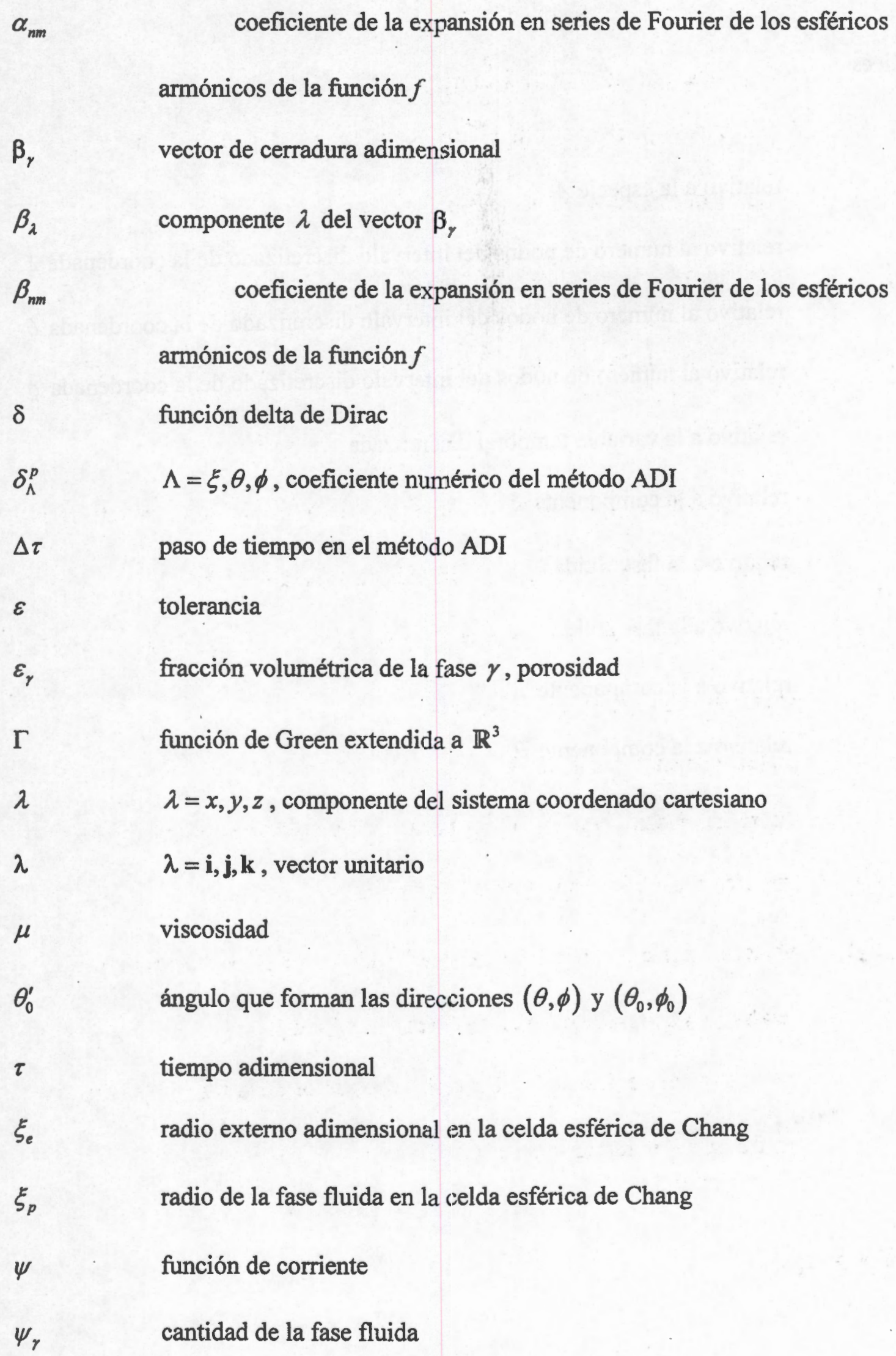


Índices

\begin{tabular}{l|l} 
A & relativo a la especie $A$ \\
\hline$i$ & relativo al número de nodos del intervalo discretizado de la coordenada $\xi$ \\
\hline$j$ & relativo al número de nodos del intervalo discretizado de la coordenada $\theta$ \\
\hline$k$ & relativo al número de nodos del intervalo discretizado de la coordenada $\phi$ \\
\hline$p$ & relativo a la variable temporal discretizada \\
$\xi \xi$ & relativo a la componente $\xi$ \\
$\gamma \gamma$ & relativo a la fase fluida \\
$\kappa$ & relativo a la fase sólida \\
$\lambda$ & relativo a la componente $\lambda$ \\
$\theta$ & relativo a la componente $\theta$
\end{tabular}




\section{Bibliografía}

Amaral Souto, H. P. and Moyne, C. 1996, Dispersion in two-dimensional periodic porous media. Part II: dispersion, Phys. Fluids 9 (8), 2253-2263.

Aris, R.: 1956, On the dispersion of solute in a fluid flow through a tube, Proc. Roy. Soc. A235, 67-77.

Bear J., Verruijt A. 1987, Modeling groundwater flow and pollution, Kluwer Acad. Publishers

Bird R. B., WE Stewart W. E., Lightfoot E. N. 2002, Transport Phenomena, John Wiley \& Sons, Inc.

Blackwell R. J., Rayne J. R., Terry W. M. 1959, Factors influencing the efficiency of miscible displacement. Petroleum Transactions AIME 216, 1-8

Brenner H. 1980, Dispersion resulting from flow through spatially periodic porous media, Phil. Trans. Roy. Soc., 297, 81-133.

Buyuktas D., Wallender W.W. 2004, Dispersion in spatially periodic porous media, Heat and Mass transfer 40, 261-270.

Carberry J. J, Bretton R. H., 1958, Axial dispersion of mass inflow through fixed beds. AIChE J 4,367-375

Chang H.C. 1982, Multi-scale analysis of effective transport in periodic heterogeneous media, Chem. Eng. Comm. 15, 83-91.

Chin, R.C.Y.; Manteuffel T.A.; De Pillis J. 1984, ADI as a preconditioning for solving the convection-diffusion equation, SIAM J. Sci. Statist. Comput. 5, 281-299

Chiu C., Walkington N. 1997, An ADI Method for Hysteretic Reaction-Diffusion Systems SIAM J. Numer. Anal. 34, 1185-1206.

Courant R., Hilbert D. 1962, Methods of mathematical physics, Vol. 1; Vol. 2, Interscience Publishers (a division of John Wiley \& Sons).

De Josselin De Jong, G., 1958, Longitudinal and transverse diffusion in granular deposits, Trans. Amer. Geophys. Union 39, 6774.

Dendy, J. E. Jr. 1977, An Alternating Direction Method for Schrödinger's Equation. SIAM J. Nume. Anal. 14, No. 6, 1028-1032 
Douglas J. Jr, Rachford H. 1960, On the numerical solution of heat conduction problems in two and three space variables, Trans. Am. Math. Soc. 82, 421-439.

Douglas J. Jr., Gunn J. 1964, A general formulation of alternating direction methods Part I. Parabolic and hyperbolic problems, Numer. Math. 6, 428-453.

Douglas J. Jr., Peaceman D. 1955 Numerical solution of two-dimensional heat flow problems, AICHE J. 1, 505-512.

Ebach EA, White RR, 1958 Mixing of fluids flowing through beds of packed solids. AIChE J 4, 161-169.

Edwards, D.A., Shapiro, M., Brenner, H. \& Shapira, M. 1991 Dispersion of inert solutes in spatially periodic, two-dimensional, model porous media. Transport in Porous Media 6, 337-358.

Edwards, M. F., and J. F. Richardson, 1968, Gas Dispersion in Packed Beds, Chem. Eng. Sci., 23, 109.

Eidsath A. B. 1981, Flow and dispersion in spatially periodic porous media : a finite element study, Master's thesis, Department of Chemical Engineering, University of California, Davis, CA.

Eidsath A. B., Carbonell R. G., Whitaker S., Herrmann L. R. 1983, Dispersion in pulsed systems III. Comparison between theory and experiments for packed beds, Chem. Eng. Sci. 38 , No. 11, 1803-1816.

Gunn, D. J., Pryce, C. 1969, Dispersion in packed beds. Trans. Instn. Chem. Engrs. 47, T341-T350.

Haberman R. 2004, Applied Partial Differential Equations: With Fourier Series and Boundary Value Problems, Pearson Prentice Hall

Haring R.E., Greenkorn R. A. 1970, A Statistical Model of a Porous Medium with Nonuniform Pores, Am. Inst. Chem. Eng. J. 16, 477-480.

Han, N-W, Bhakta, J. and Carbonell, R. G., 1985, Longitudinal and Lateral Dispersion in Packed Beds: Effects of Column Length and Particle Size Distribution, A.I.Ch.E.Journal, 31, 277-288.

Hassinger and von Rosenberg, 1968, A mathematical and experimental investigation of transverse dispersion coefficients, Soc. Petrol. Eng. J., 8, 195-204.

Horn F. J. M. 1971, Calculation of dispersion coefficients by means of moments, AICHE J. 17, No. $3,613-620$. 
Jarzebski A. B., Thullie J. W. 1896, A stable highly accurate ADI method for hyperbolic heat conduction equation, J. Comput. Phys. 63, No. 1, 236-239.

Karaa S., Zhang J. 2004, High order ADI method for solving unsteady convection-diffusion problems, J. Comput. Phys. 198, No. 1, 1 - 9.

Launder B.E., Massey T. H. 1978, The numerical predictions of viscous flow and heat transfer in tube banks, J. Heat Trans. 100, 565-571.

Marsden J. E.,Tromba A. 1998, Cálculo vectorial, México: Prentice-Hall.

Necati Ozisik M. 1994, Finite Difference Methods in Heat Transfer, CRC Press.

Neculae A., Goyeau B., Quintard M., Gobin D. 2002, Passive dispersion in dendritic structures, Materials Sci. and Eng. A323, 367-376.

Ochoa-Tapia J. A., Stroeve P., Whitaker S. 1994, Diffusive transport in two-phase media: spatially periodic models and Maxwell's theory for isotropic and anisotropic systems. Chem. Eng. Sci. 49, 709-726.

Ochoa-Tapia, J.A., Stroeve, S., Whitaker, S. 1991, Facilitated transport in porous media, Chem. Eng. Sci. 46 (2), 477-496

Peaceman, D. W. and Rachford, H.H. 1955, The Numerical Solution of Parabolic and Elliptic Differential Equations.. J. Soc.Indust. Appl. Math. 3, No. 1, 28-41.

Pfannkuch H. O. 1963, Contribution a L'Etude des Déplacements de Fluids Miscibles dans un Milieu Poreux. Rev Inst. Fr. Pétrole 18, 215-219

Plumb O. A., Whitaker S. 1988, Dispersion in heterogeneous porous media. I. Local volume averaging and large-scale averaging, Wat. Res. Research 24, No. 7, 913-926.

Quintard M., Whitaker S. 1994, Convection, Dispersion, and interfacial transport of contaminants: Homogeneous Porous media, Adv. Water Resour.. 17, 221239.

Rifai M. N. E., Kaufman W. J., Todd, D.K. 1956, Dispersion phenomena in laminar flow through porous media. University of California, Sanit Engrg Rept 3, Inst Eng Res Series 90, 1-157.

Sahraoui M., Kaviany M. 1994, Slip and no-slip temperature boundary conditions at interface of porous, plain media: Convection. International Journal of Heat and Mass Transfer 37, 1029-1044.

Sartory W. K. 1978, Adsorption on the Walls of a Cylindrical Channel, Ind. Eng. Chern. Fundarn.. 17, No. 2, 97-100.

Slattery J. C. 1999, Advanced Transport Phenomena, Cambridge University Press. 
Taylor G. 1953, Dispersion of soluble matter in solvent flowing slowly through a tube, Proc. Roy. Soc., 219, 186-203.

Wang Ting-Yuan, Lee Yu-Min, Chung-Ping Chen C. 2003, 3D Thermal-ADI- An Efficient Chip-Level Transient Thermal Simulator, Proc. Int. Symp. On Physical Design, 10-17

Weinberger H. F. 1995 ,A First Course in Partial Differential Equations with Complex Variables and Transform Methods, Dover.

Whitaker S. 1967, Diffusion and dispersion in porous media, AIChE J., 13: 420-427.

Whitaker S. 1983, Diffusion and reaction in a micropore.macropore model of a porous medium, Lat. Amer. Chem. Eng. 13, 143-183.

Whitaker S. 1999, The method of volume averaging, Kluwer Academic Publishers. 


\section{Apéndices}

\section{A.1Perfil de velocidad alrededor de una esfera de radio $R$ bajo condiciones de flujo reptante}

Equation Chapter 1 Section 1

El lugar geométrico que describe la región alrededor de la esfera es:

$$
\Omega=\{(r, \theta, \varphi): R \leq r<\infty, 0 \leq \theta<\pi, 0 \leq \varphi<2 \pi\}
$$

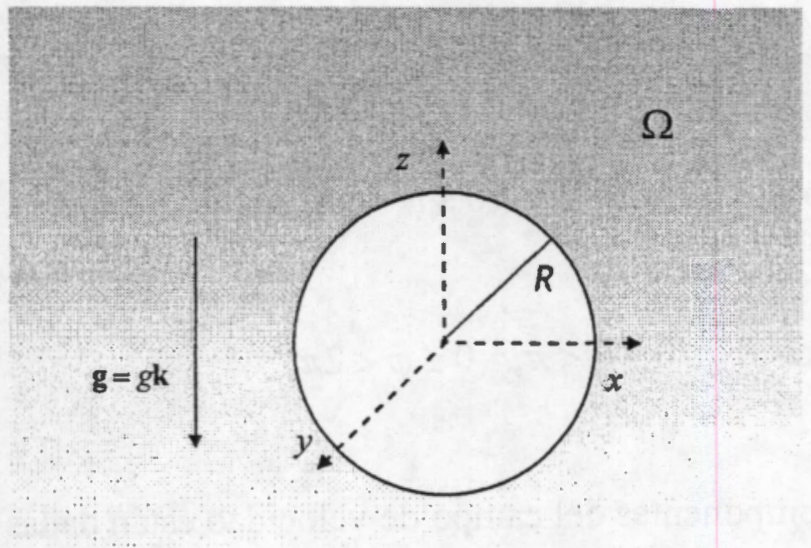

Suposiciones

3. Fluido newtoniano

4. Estado estacionario

5. Fluido incompresible

$$
\text { ( } \rho \text { constante) }
$$

6. Fluido con viscosidad constante

7. Flujo reptante bidimensional

El campo de velocidad del fluido alrededor de la esfera con flujo potencial en coordenadas esféricas es de la forma siguiente

$$
\begin{gathered}
v_{r}=v_{r}(r, \theta) \\
v_{\theta}=v_{\theta}(r, \theta) \\
v_{\varphi}=0
\end{gathered}
$$


Las condiciones de frontera en la variable radial son:

CF1: $\quad r=R, \quad \mathrm{v}=0, \quad 0 \leq \theta<\pi, \quad 0 \leq \varphi<2 \pi$

CF2: $r \rightarrow \infty, \quad \mathbf{v}=v_{\infty} \mathbf{k}, \quad 0 \leq \theta<\pi, \quad 0 \leq \varphi<2 \pi$

En términos de las componentes del campo de velocidad tenemos que

CF1a: $r=R, \quad v_{r}=0, \quad 0 \leq \theta<\pi, \quad 0 \leq \varphi<2 \pi$

CF1b: $r=R, \quad v_{\theta}=0, \quad 0 \leq \theta<\pi, \quad 0 \leq \varphi<2 \pi$

CF2a: $r \rightarrow \infty, \quad v_{r}=v_{\infty} \cos \theta, \quad 0 \leq \theta<\pi, \quad 0 \leq \varphi<2 \pi$

CF2b: $r \rightarrow \infty, \quad v_{\theta}=-\nu_{\infty} \operatorname{sen} \theta, \quad 0 \leq \theta<\pi, \quad 0 \leq \varphi<2 \pi$

En términos de la función de corriente las componentes del campo de velocidad están dadas por:

$$
\begin{aligned}
& v_{r}=\frac{1}{r^{2} \operatorname{sen} \theta} \frac{\partial \psi}{\partial \theta} \\
& v_{\theta}=-\frac{1}{r \operatorname{sen} \theta} \frac{\partial \psi}{\partial r}
\end{aligned}
$$

En estado estacionario bajo la suposición de flujo reptante, la ecuación de Navier-Stokes en términos de la función de corriente está dada por:

$$
\mathrm{E}^{4} \psi=0
$$

Donde el operador $E^{2}$ está dado por 


$$
E^{2}=\frac{\partial^{2}}{\partial r^{2}}+\frac{\operatorname{sen} \theta}{r^{2}} \frac{\partial}{\partial \theta}\left(\frac{1}{\operatorname{sen} \theta} \frac{\partial}{\partial \theta}\right)
$$

De las condiciones de frontera $\mathrm{CF} 1$ y $\mathrm{CF} 2$ tenemos que las condiciones de frontera para la función de frontera para la función de corriente son

$$
\begin{gathered}
\text { CF } 1 \mathrm{a}_{\varphi}: r=R, \quad \frac{1}{r^{2} \operatorname{sen} \theta} \frac{\partial \psi}{\partial \theta}=0, \quad 0 \leq \theta<\pi, \quad 0 \leq \varphi<2 \pi \\
\mathrm{CF} 1 \mathrm{~b}_{\varphi}: r=R, \quad \frac{1}{r \operatorname{sen} \theta} \frac{\partial \psi}{\partial r}=0, \quad 0 \leq \theta<\pi, \quad 0 \leq \varphi<2 \pi \\
\mathrm{CF} 2 \mathrm{a}_{\varphi}: r \rightarrow \infty, \quad v_{r}=\frac{1}{r^{2} \operatorname{sen} \theta} \frac{\partial \psi}{\partial \theta} \rightarrow v_{\infty} \cos \theta, \quad 0 \leq \theta<\pi, \quad 0 \leq \varphi<2 \pi
\end{gathered}
$$

De la condición de frontera tenemos que CF2a $\mathrm{a}_{\varphi}$ tenemos que

$$
r \rightarrow \infty, \psi=v_{\infty} r^{2} \int_{0}^{\theta} \operatorname{sen} \theta \cos \theta d \theta
$$

Resolviendo la integral sabiendo que la función de corriente tenemos que

$$
\begin{gathered}
r \rightarrow \infty, \theta=0, \psi \rightarrow 0 \\
r \rightarrow \infty, \psi=\frac{v_{\infty} r^{2}}{2} \operatorname{sen}^{2} \theta
\end{gathered}
$$

La condición de frontera CF2a $\mathrm{a}_{\varphi}$ sugiere que la expresión de la función de corriente esté dada por 


$$
\psi(r, \theta)=f(r) \operatorname{sen}^{2} \theta
$$

El operador $E^{2}$ está dado por

$$
\mathrm{E}^{2} \psi=\left(\frac{\partial^{2} f(r)}{\partial r^{2}}-2 \frac{f(r)}{r^{2}}\right) \operatorname{sen}^{2} \theta
$$

Aplicando dos veces el operador $\mathrm{E}^{2}$ tenemos que

$$
\mathrm{E}^{4} \psi=\left(\frac{\partial^{4} f(r)}{\partial r^{4}}-4 \frac{1}{r^{2}} \frac{\partial^{2} f(r)}{\partial r^{2}}+\frac{8}{r^{3}} \frac{\partial f(r)}{\partial r}-8 \frac{f(r)}{r^{4}}\right) \operatorname{sen}^{2} \theta
$$

De esta manera de la ecuación de Navier-Stokes para la función de corriente (A.1.6) tenemos que la ecuación diferencial ordinaria para la función $\mathrm{f}$ está dada por

$$
\frac{\partial^{4} f(r)}{\partial r^{4}}-4 \frac{1}{r^{2}} \frac{\partial^{2} f(r)}{\partial r^{2}}+\frac{8}{r^{3}} \frac{\partial f(r)}{\partial r}-8 \frac{f(r)}{r^{4}}=0
$$

De las condiciones de frontera $C F 1 a_{\varphi}, C F 1 b_{\varphi}$ y CF2 $a_{\varphi}$ tenemos que las condiciones de frontera para la función de corriente son

$$
\begin{gathered}
\text { CF1 a }: r=R, f(r)=0 \\
\text { CF1 bf: } r=R, \quad \frac{\partial f(r)}{\partial r}=0 \\
C F 2_{\mathrm{f}: ~} \rightarrow \infty, \quad \frac{f(r)}{r^{2}}=\frac{1}{2} v_{\infty}
\end{gathered}
$$


La ecuación diferencial (A.1.17) es una ecuación de Euler, por lo que se propone como solución la función siguiente

$$
f(r)=r^{\alpha}
$$

Las derivadas de al función $\mathrm{f}$ se presentan a continuación

$$
\begin{gathered}
\frac{d f(r)}{d r}=\alpha r^{\alpha-1} \\
\frac{d^{2} f(r)}{d r^{2}}=\alpha(\alpha-1) r^{\alpha-2} \\
\frac{d^{3} f(r)}{d r^{3}}=\alpha(\alpha-1)(\alpha-2) r^{\alpha-3} \\
\frac{d^{4} f(r)}{d r^{4}}=\alpha(\alpha-1)(\alpha-2)(\alpha-3) r^{\alpha-4}
\end{gathered}
$$

Sustituyendo las expresiones de las derivadas dadas por las ecuaciones (A.1.22)-(A.1.25) en la ecuación diferencial (A.1.17) se tiene que

$$
\dot{\alpha}(\alpha-1)(\alpha-2)(\alpha-3) r^{\alpha-4}-4 \alpha(\alpha-1) r^{\alpha-4}+8 \alpha r^{\alpha-4}-8 r^{\alpha-4}=0
$$

Factorizando se tiene que

$$
(\alpha-1)(\alpha-2)(\alpha-4)(\alpha+1)=0
$$

De esta manera se tiene que la solución general a la ecuación diferencial (A.1.17) está dada por 


$$
f(r)=\frac{A}{r}+B r+C r^{2}+D r^{4}
$$

La derivada de la función $f$ dada por la ecuación (A.1.28) resulta estar dada por

$$
\frac{d f(r)}{d r}=-\frac{A}{r^{2}}+B+2 C r+4 D r^{3}
$$

De la condición de frontera $\mathrm{CF} 1_{\mathrm{f}}$ se tiene que

$$
0=\frac{A}{R}+B R+C R^{2}+D R^{4}
$$

De la condición de frontera $\mathrm{CF} 1_{\mathrm{f}}$ se tiene que

$$
0=-\frac{A}{R^{2}}+B+2 C R+4 D R^{3}
$$

De la expresión de $f$ se tiene que

$$
\frac{f(r)}{r^{2}}=\frac{A}{r^{3}}+\frac{B}{r}+C+D r^{2}
$$

Como de la condición de frontera CF2a $\mathrm{a}_{\mathrm{f}}$ se debe cumplir que

$$
\lim _{r \rightarrow \infty} \frac{f(r)}{r^{2}}=\frac{v_{\infty}}{2}
$$

Entonces

$$
\begin{gathered}
D=0 \\
C=\frac{\nu_{\infty}}{2}
\end{gathered}
$$

De las ecuaciones (1.) y (1.) se tiene que 


$$
\begin{aligned}
& A=\frac{\nu_{\infty}}{4} R^{3} \\
& B=-\frac{3 v_{\infty}}{4} R
\end{aligned}
$$

La expresión de $f$ resulta estar dada por

$$
f(r)=\frac{v_{\infty}}{2}\left(\frac{R^{3}}{2 r}-\frac{3}{2} R r+r^{2}\right)
$$

La expresión de la función de corriente es

$$
\psi(r, \theta)=-\frac{v_{\infty}}{2}\left(\frac{R^{3}}{2 r}-\frac{3}{2} R r+r^{2}\right) \operatorname{sen}^{2} \theta
$$

Las derivadas parciales de la función de corriente son

$$
\begin{gathered}
\frac{\partial}{\partial r} \psi(r, \theta)=\frac{\nu_{\infty}}{2}\left(-\frac{R^{3}}{2 r^{2}}-\frac{3}{2} R+2 r\right) \operatorname{sen}^{2} \theta \\
\frac{\partial}{\partial \theta} \psi(r, \theta)=-v_{\infty}\left(\frac{R^{3}}{2 r}-\frac{3}{2} R r+r^{2}\right) \operatorname{sen} \theta \cos \theta
\end{gathered}
$$

De esta manera las componentes no nulas del campo de velocidad son

$$
v_{r}=v_{\infty}\left(1-\frac{3}{2} \frac{R}{r}+\frac{1}{2}\left(\frac{R}{r}\right)^{3}\right) \cos \theta
$$




$$
v_{\theta}=-v_{\infty}\left(1-\frac{3}{4} \frac{R}{r}-\frac{1}{4}\left(\frac{R}{r}\right)^{3}\right) \operatorname{sen} \theta
$$




\section{A.2Solución a la ecuación de Poisson con condiciones de frontera no- homogéneas usando la función de Green en la celda de Chang con geometría esférica}

Equation Chapter 1 Section 2

El problema de contorno descrito por la ecuación de Poisson (4.3.26)-(4.3.28) en coordenadas esféricas está dado por

$$
\begin{array}{r}
\frac{1}{\xi^{2}} \frac{\partial}{\partial \xi}\left(\xi^{2} \frac{\partial B_{\lambda}}{\partial \xi}\right)+\frac{1}{\xi^{2} \operatorname{sen} \theta} \frac{\partial}{\partial \theta}\left(\operatorname{sen} \theta \frac{\partial B_{\lambda}}{\partial \theta}\right)+\frac{1}{\xi^{2} \operatorname{sen}^{2} \theta} \frac{\partial^{2} B_{\lambda}}{\partial \phi^{2}}=-F(\xi, \theta, \phi) \\
(\xi, \theta, \phi) \in\left(\xi_{p}, \xi_{e}\right) \times(0, \pi) \times(0,2 \pi)
\end{array}
$$

Donde

$$
F(\xi, \theta, \phi)=P e \tilde{U}_{\lambda}(\xi, \theta, \phi)
$$

El problema de contorno está sujeto a las condiciones de frontera de tipo Neumann en la interfase y Dirichlet en el radio externo, éstas se escriben como sigue

$$
\begin{gathered}
\frac{\partial B_{\lambda}}{\partial \xi}\left(\xi_{p}, \theta, \phi\right)=f(\theta, \phi) \\
B_{\lambda}\left(\xi_{e}, \theta, \phi\right)=0
\end{gathered}
$$

Desarrollando la función $F$ en series de Fourier de $P_{n}^{m}(\cos \theta) \cos m \phi$ y $P_{n}^{m}(\cos \theta) \operatorname{sen} m \phi$ (esféricos armónicos), esto al tomar su transformada finita de Fourier:

$$
F(\xi, \theta, \phi)=\sum_{n=0}^{\infty}\left\{\frac{A_{n 0}(\xi)}{2} P_{n}(\cos \theta)+\sum_{m=1}^{n}\left[A_{n m}(\xi) \cos m \phi+B_{n m}(\xi) \operatorname{sen} m \phi\right] P_{n}^{m}(\cos \theta)\right\}
$$


Los coeficientes desconocidos se determinan a partir de la propiedad de ortogonalidad de los polinomios de Legendre $P_{n}^{m}(\cos \theta)$, y de las funciones trigonométricas $\cos m \phi$ y sen $m \phi$ y están dados por las siguientes expresiones

$$
\begin{aligned}
& A_{n m}(\xi)=\frac{(2 n+1)(n-m) !}{2 \pi(n+m) !} \int_{0}^{2 \pi} \int_{0}^{\pi} F(\xi, \theta, \phi) P_{n}^{m}(\cos \theta) \cos m \phi \operatorname{sen} \theta d \theta d \phi \\
& B_{n m}(\xi)=\frac{(2 n+1)(n-m) !}{2 \pi(n+m) !} \int_{0}^{2 \pi} \int_{0}^{\pi} F(\xi, \theta, \phi) P_{n}^{m}(\cos \theta) \operatorname{sen} m \phi \operatorname{sen} \theta d \theta d \phi
\end{aligned}
$$

Si el problema de valores a la frontera tiene solución, entonces su expansión en series de Fourier en $P_{n}^{m}(\cos \theta) \cos m \phi$ y $P_{n}^{m}(\cos \theta) \operatorname{sen} m \phi$ es

$$
B_{\lambda}(\xi, \theta, \phi)=\sum_{n=0}^{\infty}\left\{\frac{a_{n 0}(\xi)}{2} P_{n}(\cos \theta)+\sum_{m=1}^{n}\left[a_{n m}(\xi) \cos m \phi+b_{n m}(\xi) \operatorname{sen} m \phi\right] P_{n}^{m}(\cos \theta)\right\}
$$

Los coeficientes se encuentran a través de las siguientes ecuaciones

$$
\begin{aligned}
& a_{n m}(\xi)=\frac{(2 n+1)(n-m) !}{2 \pi(n+m) !} \int_{0}^{2 \pi} \int_{0}^{\pi} B_{\lambda}(\xi, \theta, \phi) P_{n}^{m}(\cos \theta) \cos m \phi \operatorname{sen} \theta d \theta d \phi \\
& b_{n m}(\xi)=\frac{(2 n+1)(n-m) !}{2 \pi(n+m) !} \int_{0}^{2 \pi} \int_{0}^{\pi} B_{\lambda}(\xi, \theta, \phi) P_{n}^{m}(\cos \theta) \operatorname{sen} m \phi \operatorname{sen} \theta d \theta d \phi
\end{aligned}
$$

De la condición de frontera en $\xi=\xi_{e}$, se tiene que

$$
\begin{aligned}
& a_{n m}\left(\xi_{e}\right)=0, n \geq 0, m \geq 0 \text { y } m, n \in \mathbb{N} \\
& b_{n m}\left(\xi_{e}\right)=0, n \geq 1, m \geq 1 \text { y } m, n \in \mathbb{N}
\end{aligned}
$$


Derivando la función $B_{\lambda}$ dada por la ecuación (A.2.7) con respecto a $\xi$ y aplicando la condición de frontera en $\xi=\xi_{p}$, se encuentra la siguiente expresión

$$
f(\theta, \phi)=\sum_{n=0}^{\infty} \frac{a_{n 0}^{\prime}\left(\xi_{p}\right)}{2} P_{n}(\cos \theta)+\sum_{n=1}^{\infty} \sum_{m=1}^{n}\left[a_{n m}^{\prime}\left(\xi_{p}\right) \cos m \phi+b_{n m}^{\prime}\left(\xi_{p}\right) \operatorname{sen} m \phi\right] P_{n}^{m}(\cos \theta)
$$

Por otro lado, se tiene que la expansión en series de Fourier en términos de los esféricos armónicos $\left(P_{n}^{m}(\cos \theta) \cos m \phi\right.$ y $\left.P_{n}^{m}(\cos \theta) \operatorname{sen} m \phi\right)$ de la función $f$ es

$$
f(\theta, \phi)=\sum_{n=0}^{\infty}\left\{\frac{\alpha_{n 0}}{2} P_{n}(\cos \theta)+\sum_{m=1}^{n}\left[\alpha_{n m} \cos m \phi+\beta_{m m} \operatorname{sen} m \phi\right] P_{n}^{m}(\cos \theta)\right\}
$$

Donde los coeficientes $\alpha_{n m}$ y $\beta_{n m}$ están dados por

$$
\begin{aligned}
& \alpha_{n m}=\frac{(2 n+1)(n-m) !}{2 \pi(n+m) !} \int_{0}^{2 \pi} \int_{0}^{\pi} f(\theta, \phi) P_{n}^{m}(\cos \theta) \cos m \phi \operatorname{sen} \theta d \theta d \phi \\
& \beta_{n m}=\frac{(2 n+1)(n-m) !}{2 \pi(n+m) !} \int_{0}^{2 \pi} \int_{0}^{\pi} f(\theta, \phi) P_{n}^{m}(\cos \theta) \operatorname{sen} m \phi \operatorname{sen} \theta d \theta d \phi
\end{aligned}
$$

Por identificación, se concluye que

$$
\begin{array}{ll}
a_{n m}^{\prime}\left(\xi_{p}\right)=\alpha_{n m}, & n \geq 0, m \geq 0 \text { y } m, n \in \mathbb{N} \\
b_{n m}^{\prime}\left(\xi_{p}\right)=\beta_{n m}, & n \geq 1, m \geq 1 \text { y } m, n \in \mathbb{N}
\end{array}
$$

Tomando la transformada finita de Fourier de la ecuación de Poisson e integrando por partes, se encuentra que los coeficientes $a_{n m}(\xi)$ y $b_{n m}(\xi)$ están dados por los siguientes problemas de valores a la frontera 


$$
\begin{aligned}
& \frac{d}{d \xi}\left(\xi^{2} \frac{d a_{n m}}{d \xi}\right)-n(n+1)(\xi) a_{n m}=-\xi^{2} A_{n m} \\
& \left.\begin{array}{l}
a_{n m}^{\prime}\left(\xi_{p}\right)=\alpha_{n m} \\
a_{n m}\left(\xi_{e}\right)=0
\end{array}\right\} n \geq 0, m \geq 0 \text { y } m, n \in \mathbb{N} \\
& \frac{d}{d \xi}\left(\xi^{2} \frac{d b_{n m}}{d \xi}\right)-n(n+1)(\xi) b_{n m}=-\xi^{2} B_{n m} \\
& \left.\begin{array}{l}
b_{n m}^{\prime}\left(\xi_{p}\right)=\beta_{n m} \\
b_{n m}\left(\xi_{e}\right)=0
\end{array}\right\} n \geq 1, m \geq 1 \text { y } m, n \in \mathbb{N}
\end{aligned}
$$

Las ecuaciones diferenciales ordinarias homogéneas asociadas para $a_{n m}$ y $b_{n m}$ son ecuaciones de Cauchy-Euler, cuyas soluciones son una combinación lineal de las funciones $v_{1, n}(\xi)=\xi^{n}$ y $v_{2, n}(\xi)=\xi^{-(n+1)}$, que son linealmente independientes.

Como ambos problemas de contorno tienen la misma ecuación diferencial ordinaria, están definidos en el mismo dominio $\left[\xi_{p}, \xi_{e}\right]$ y presentan el mismo tipo de condiciones de frontera (Neumann-Dirichlet), entonces, la función de Green asociada es la misma para dichos problemas. El desarrollo para la obtención de la solución de este problema en términos de la función de Green, se encuentra en los apéndices A.3 y A.4. Entonces, las soluciones de los problemas en términos de la función de Green son

$$
\begin{aligned}
& a_{n m}(\xi)=\int_{\xi_{p}}^{\xi} g_{n}\left(\xi, \xi_{0}\right) A_{n m}\left(\xi_{0}\right) \xi_{0}^{2} d \xi_{0}+a_{n m}^{\prime}\left(\xi_{p}\right) g\left(\xi, \xi_{p}\right) \\
& b_{n m}(\xi)=\int_{\xi_{p}}^{\xi} g_{n}\left(\xi, \xi_{0}\right) B_{n m}\left(\xi_{0}\right) \xi_{0}^{2} d \xi_{0}+b_{n m}^{\prime}\left(\xi_{p}\right) g\left(\xi, \xi_{p}\right)
\end{aligned}
$$

En el apéndice A.4 se presenta el desarrollo para la obtención de la función de Green, sujeta a las condiciones de frontera homogéneas asociadas a los problemas de contorno dados por 
las ecuaciones (A.2.14)-(A.2.15) y (A.2.16)-(A.2.17), en términos del conjunto fundamental de solucionès homogéneas de la ecuación diferencial ordinaria. Así, la función de Green para estos problemas de valores a la frontera es

$$
g_{n}\left(\xi, \xi_{0}\right)=\left\{\begin{array}{l}
\frac{1}{(2 n+1) \xi_{e}} \frac{n \xi_{p}^{2 n+1}+(n+1) \xi_{0}^{2 n+1}}{n \xi_{p}^{2 n+1}+(n+1) \xi_{e}^{2 n+1}}\left(\frac{\xi_{0}}{\xi_{e}}\right)^{n+1}\left[\left(\frac{\xi}{\xi_{e}}\right)^{n}-\left(\frac{\xi}{\xi_{e}}\right)^{-n-1}\right] \xi_{0} \leq \xi \\
\frac{1}{(2 n+1) \xi_{e}} \frac{n \xi_{p}^{2 n+1}+(n+1) \xi^{2 n+1}}{n \xi_{p}^{2 n+1}+(n+1) \xi_{e}^{2 n+1}}\left(\frac{\xi}{\xi_{e}}\right)^{n+1}\left[\left(\frac{\xi_{0}}{\xi_{e}}\right)^{n}-\left(\frac{\xi_{0}}{\xi_{e}}\right)^{-n-1}\right] \xi \leq \xi_{0}
\end{array}\right.
$$

Sustituyendo las definiciones de $A_{n m}$ y $B_{n m}$ dadas por las expresiones (A.2.6) en las ecuaciones que determinan los coeficientes $a_{n m}$ (A.2.18) y $b_{n m}$ (A.2.19), y usando el teorema de Fubini para poder intercambiar el orden de integración y cambiando las variables mudas de integración, se obtiene

$$
\begin{array}{r}
a_{n m}(\xi)=\frac{(2 n+1)(n-m) !}{2 \pi(n+m) !} \int_{0}^{2 \pi} \int_{0}^{\pi} \int_{\xi_{p}} g_{n}\left(\xi, \xi_{0}\right) F\left(\xi_{0}, \theta_{0}, \phi_{0}\right) P_{n}^{m}\left(\cos \theta_{0}\right) \cos m \phi_{0} \xi_{0}^{2} \operatorname{sen} \theta d \xi_{0} d \theta_{0} d \phi_{0} \\
+a_{m m}^{\prime}\left(\xi_{p}\right) g_{n}\left(\xi, \xi_{p}\right) \\
(\text { A.2.21) } \\
b_{n m}(\xi)=\frac{(2 n+1)(n-m) !}{2 \pi(n+m) !} \int_{0}^{2 \pi} \int_{0}^{\pi} \int_{\xi_{p}}^{\pi} g_{n}\left(\xi, \xi_{0}\right) F\left(\xi_{0}, \theta_{0}, \phi_{0}\right) P_{n}^{m}\left(\cos \theta_{0}\right) \operatorname{sen} m \phi \xi_{0}^{2} \operatorname{sen} \theta_{0} d \xi_{0} d \theta_{0} d \phi_{0} \\
+b_{n m}^{\prime}\left(\xi_{p}\right) g\left(\xi, \xi_{p}\right)
\end{array}
$$

Sustituyendo estas expresiones en la serie de Fourier que define a $B_{\lambda}$ dada por la ecuación (A.2.7), y dada la linealidad del operador integral es posible intercambiar la integración y la suma, resultando la siguiente expresión 


$$
\begin{array}{r}
B_{\lambda}(\xi, \theta, \phi)=\int_{0}^{2 \pi} \int_{0}^{\pi} \int_{\xi_{p}}^{\xi} F\left(\xi_{0}, \theta_{0}, \phi_{0}\right) \sum_{n=0}^{\infty} g_{n}\left(\xi, \xi_{0}\right) K_{n}\left(\theta, \phi ; \theta_{0}, \phi_{0}\right) \xi_{0}^{2} \operatorname{sen} \theta_{0} d \xi_{0} d \theta_{0} d \phi_{0} \\
+\sum_{n=0}^{\infty} g_{n}\left(\xi, \xi_{p}\right)\left\{\frac{a_{n 0}^{\prime}(1)}{2} P_{n}(\cos \theta)+\sum_{m=1}^{n}\left[a_{n m}^{\prime}\left(\xi_{p}\right) \cos m \phi+b_{n m}^{\prime}\left(\xi_{p}\right) \operatorname{sen} m \phi\right] P_{n}^{m}(\cos \theta)\right\}
\end{array}
$$

Donde

$$
\begin{aligned}
K_{n}\left(\theta, \phi ; \theta_{0}, \phi_{0}\right)= & \frac{(2 n+1)}{4 \pi} P_{n}(\cos \theta) P_{n}\left(\cos \theta_{0}\right) \\
& +\sum_{m=1}^{n} \frac{(2 n+1)(n-m) !}{2 \pi(n+m) !} P_{n}^{m}(\cos \theta) P_{n}^{m}\left(\cos \theta_{0}\right) \cos m\left(\phi-\phi_{0}\right)
\end{aligned}
$$

El kernel cumple también la siguiente igualdad

$$
K_{n}\left(\theta, \phi ; \theta_{0}, \phi_{0}\right)=\frac{2 n+1}{4 \pi} P_{n}\left(\cos \theta_{0}^{\prime}\right)
$$

Donde $\theta_{0}^{\prime}$ es el ángulo formado por las direcciones $(\theta, \phi)$ y $\left(\theta_{0}, \phi_{0}\right)$ y está definido por

$$
\cos \theta_{0}^{\prime}=\cos \theta \cos \theta_{0}+\operatorname{sen} \theta \operatorname{sen} \theta_{0} \cos \left(\phi-\phi_{0}\right)
$$

Sustituyendo el valor de las derivadas de los coeficientes $a_{n m}$ y $b_{n m}$ evaluadas en $\xi=\xi_{p}$, dados por las ecuaciones (A.2.13) en la expresión de la componente $\lambda$ del vector de cerradura (A.2.7), se obtiene que 


$$
\begin{array}{r}
B_{\lambda}(\xi, \theta, \phi)=\int_{0}^{2 \pi} \int_{0}^{\pi} \int_{\xi_{p}} F\left(\xi_{0}, \theta_{0}, \phi_{0}\right) \sum_{n=0}^{\infty} g_{n}\left(\xi, \xi_{0}\right) K_{n}\left(\theta, \phi ; \theta_{0}, \phi_{0}\right) \xi_{0}^{2} \operatorname{sen} \theta_{0} d \xi_{0} d \theta_{0} d \phi_{0} \\
+\int_{0}^{2 \pi} \int_{0}^{\pi} f(\theta, \phi) \sum_{n=0}^{\infty} g_{n}\left(\xi, \xi_{p}\right) K_{n}\left(\theta, \phi ; \theta_{0}, \phi_{0}\right) \operatorname{sen} \theta d \theta d \phi
\end{array}
$$

La definición de la función de Green es la siguiente

$$
G\left(\xi, \theta, \phi ; \xi_{0}, \theta_{0}, \phi_{0}\right)=\sum_{n=0}^{\infty} g_{n}\left(\xi, \xi_{0}\right) K_{n}\left(\theta, \phi ; \theta_{0}, \phi_{0}\right)
$$

O en términos del ángulo $\theta_{0}^{\prime}$ como

$$
G\left(\xi, \theta, \phi ; \xi_{0}, \theta_{0}, \phi_{0}\right)=\frac{1}{4 \pi} \sum_{n=0}^{\infty}(2 n+1) g_{n}\left(\xi, \xi_{0}\right) P_{n}\left(\cos \theta_{0}^{\prime}\right)
$$

Teniendo en cuenta la definición de la función de Green (4.6.5), la solución a la ecuación de Poisson se reduce a

$$
\begin{array}{r}
B_{\lambda}(\xi, \theta, \phi)=\int_{0}^{2 \pi} \int_{0}^{\pi} \int_{\xi_{p}}^{\xi_{0}} F\left(\xi_{0}, \theta_{0}, \phi_{0}\right) G\left(\xi, \theta, \phi ; \xi_{0}, \theta_{0}, \phi_{0}\right) \xi_{0}^{2} \operatorname{sen} \theta_{0} d \xi_{0} d \theta_{0} d \phi_{0} \\
+\int_{0}^{2 \pi} \int_{0}^{\pi} f(\theta, \phi) G\left(\xi, \theta, \phi ; \xi_{p}, \theta_{0}, \phi_{0}\right) \operatorname{sen} \theta d \theta d \phi
\end{array}
$$

Es importante mencionar la identidad siguiente que, es resultado de la función generadora de los polinomios de Legendre

$$
\frac{1}{\xi_{e}} \sum_{n=0}^{\infty}\left(\frac{\xi_{0}}{\xi_{e}}\right)^{n} P_{n}(\cos \theta)=\left(\xi_{0}^{2}+\xi_{e}^{2}-2 \xi_{0} \xi_{e} \cos \theta\right)^{-\frac{1}{2}}
$$


Finalmente, la componente $\lambda$ del vector de cerradura en términos de la función de Green $\operatorname{con} F\left(\xi_{0}, \theta_{0}, \phi_{0}\right)=P e \tilde{U}_{\lambda}\left(\xi_{0}, \theta_{0}, \phi_{0}\right)$ es

$$
\begin{array}{r}
B_{\lambda}(\xi, \theta, \phi)=P e \int_{0}^{2 \pi} \int_{0}^{\pi} \int_{\xi_{p}}^{\pi} \tilde{U}_{\lambda}\left(\xi_{0}, \theta_{0}, \phi_{0}\right) G\left(\xi, \theta, \phi ; \xi_{0}, \theta_{0}, \phi_{0}\right) \xi_{0}^{2} \operatorname{sen} \theta_{0} d \xi_{0} d \theta_{0} d \phi_{0} \\
+\int_{0}^{2 \pi} \int_{0}^{\pi} f(\theta, \phi) G\left(\xi, \theta, \phi ; \xi_{p}, \theta_{0}, \phi_{0}\right) \operatorname{sen} \theta d \theta d \phi
\end{array}
$$

\section{Independencia axial simétrica de la solución del problema de cerradura}

A continuación se demuestra que, sí el término no homogéneo del problema de cerradura es axialmente simétrico, al igual que la condición de frontera en $\xi=\xi_{p}$, es decir, que es independiente de la variable $\phi$, entonces la solución al problema de cerradura también es axialmente simétrica.

Como el término no homogéneo en la ecuación (4.6.1) sólo es función de $\xi_{0}$ y $\theta_{0}$, es decir, $F=F\left(\xi_{0}, \theta_{0}\right)$, entonces por el teorema de Fubini y por la linealidad de la integral, la integral en la variable $\phi_{0}$ de la solución del problema de cerradura puede intercambiarse de tal forma que el integrando de esta sea la función n-ésima de Green, $G_{n}\left(\xi, \theta, \phi ; \xi_{0} \theta_{0}, \phi_{0}\right)=g_{n}\left(\xi ; \xi_{0}\right) K_{n}\left(\theta, \phi ; \theta_{0}, \phi_{0}\right)$, y como dicha función puede verse como el producto de una función que únicamente depende de la variable radial por una que depende sólo de las variables angulares que corresponde al kernel $K_{n}\left(\theta, \phi ; \theta_{0}, \phi_{0}\right)$, entonces la integral en la variable azimutal tiene solamente por integrando el kernel dado por la ecuación (4.6.7). Esta integral tiene por resultado,

$$
\int_{0}^{2 \pi} K_{n}\left(\theta, \phi ; \theta_{0}, \phi_{0}\right) d \phi_{0}=\frac{2 n+1}{2} P_{n}(\cos \theta) P_{n}\left(\cos \theta_{0}\right)
$$


De esta manera, la solución al problema de cerradura dada por la ecuación (4.6.4) está dada por:

$$
\begin{array}{r}
B_{\lambda}(\xi, \theta)=\int_{0}^{\pi} \int_{\xi_{p}}^{\xi} F\left(\xi_{0}, \theta_{0}\right) \sum_{n=0}^{\infty} \frac{2 n+1}{2} g_{n}\left(\xi, \xi_{0}\right) P_{n}(\cos \theta) P_{n}\left(\cos \theta_{0}\right) \xi_{0}^{2} \operatorname{sen} \theta_{0} d \xi_{0} d \theta_{0} \\
+\sum_{n=0}^{\infty} \frac{\alpha_{n 0}}{2} g_{n}\left(\xi, \xi_{p}\right) P_{n}(\cos \theta)
\end{array}
$$

En este caso se puede definir la función de Green como

$$
G\left(\xi, \theta, \phi ; \xi_{0}, \theta_{0}, \phi_{0}\right)=\frac{1}{2} \sum_{n=0}^{\infty}(2 n+1) g_{n}\left(\xi, \xi_{0}\right) P_{n}(\cos \theta) P_{n}\left(\cos \theta_{0}\right)
$$

De acuerdo a la definición anterior (4.6.12), la solución al problema de cerradura en términos de la función de Green es

$$
\begin{array}{r}
B_{\lambda}(\xi, \theta)=P e \int_{0}^{\pi} \int_{\xi_{p}}^{\xi_{\xi_{0}}} \tilde{U}_{\lambda}\left(\xi_{0}, \theta_{0}\right) G\left(\xi, \theta, \phi ; \xi_{0}, \theta_{0}, \phi_{0}\right) \xi_{0}^{2} \operatorname{sen} \theta_{0} d \xi_{0} d \theta_{0} \\
+\int_{0}^{\pi} f\left(\xi_{p}, \theta_{0}\right) G\left(\xi, \theta_{;} \xi_{p}, \theta_{0}\right) \operatorname{sen} \theta_{0} d \theta_{0}
\end{array}
$$

Así, queda demostrado que la solución sólo es función de la variable radial y azimutal, es decir, es axialmente simétrica. 


\section{A.3Solución a la ecuación diferencial ordinaria de segundo orden con} condiciones de frontera de tercer tipo usando la función de Green

Equation Chapter 1 Section 3

Consideremos el problema de valores a la frontera dado por

$$
\frac{d}{d \xi}\left(p \frac{d w}{d \xi}\right)+q w=-f
$$

Sujeto a las siguientes condiciones de frontera

$$
\begin{aligned}
& \frac{d w(1)}{d \xi}=\alpha \\
& w\left(\xi_{e}\right)=0
\end{aligned}
$$

Sea $L$ el operador diferencial dado por

$$
L \equiv \frac{d}{d \xi}\left(p \frac{d}{d \xi}\right)+q
$$

Sean $A$ y $B$ funciones reales definidas en $\left[1, \xi_{e}\right]$, usando la fórmula de Green tenemos que

$$
\int_{1}^{\xi}[A L(B)-B L(A)] d \xi=p\left[A \frac{d B}{d \xi}-B \frac{d A}{d \xi}\right]_{1}^{\xi_{e}}
$$

Para demostrar la reciprocidad, en la fórmula de Green hacemos $A(\xi)=G\left(\xi, \xi_{0}\right)$ y $B(\xi)=G\left(\xi, \xi_{1}\right)$, las cuales satisfacen que $L(A)=\delta\left(\xi-\xi_{0}\right)$ y $L(B)=\delta\left(\xi-\xi_{1}\right)$ con condiciones asociadas de frontera homogéneas, 


$$
\int_{1}^{\xi_{\xi}}\left[G\left(\xi, \xi_{0}\right) \delta\left(\xi-\xi_{1}\right)-G\left(\xi, \xi_{1}\right) \delta\left(\xi-\xi_{0}\right)\right] d \xi=0
$$

De las propiedades de la función delta de Dirac, obtenemos la reciprocidad de la función de Green,

$$
G\left(\xi_{1}, \xi_{0}\right)=G\left(\xi_{0}, \xi_{1}\right)
$$

Para determinar la función de Green del problema de contorno, hagamos en la ecuación de la fórmula de Green, $A(\xi)=w(\xi)$ y $B(\xi)=G\left(\xi, \xi_{0}\right)$. Estas funciones satisfacen los siguientes problemas de valores a la frontera

$$
\begin{array}{lll}
L(w)=-f & \text { (A.3.8) } & L(G)=\delta\left(\xi-\xi_{0}\right) \\
\frac{d w(1)}{d \xi}=\alpha & \text { (A.3.10) } & \frac{d G(\xi, 1)}{d \xi}=0 \\
w\left(\xi_{e}\right)=0 & \text { (A.3.12) } & G\left(\xi, \xi_{e}\right)=0
\end{array}
$$

De esta manera tenemos que

$$
\int_{1}^{\xi}\left[w(\xi) \delta\left(\xi-\xi_{0}\right)+G\left(\xi, \xi_{0}\right) f(\xi)\right] d \xi=p\left[w(\xi) \frac{d G\left(\xi, \xi_{0}\right)}{d \xi}-G\left(\xi, \xi_{0}\right) \frac{d w(\xi)}{d \xi}\right]_{1}^{\xi_{e}}
$$

De las propiedades de la función delta de Dirac, obtenemos 


$$
w\left(\xi_{0}\right)=-\int_{1}^{\xi_{0}} G\left(\xi, \xi_{0}\right) f(\xi) d \xi+p\left[w(\xi) \frac{d G\left(\xi, \xi_{0}\right)}{d \xi}-G\left(\xi, \xi_{0}\right) \frac{d w(\xi)}{d \xi}\right]_{1}^{\xi_{0}}
$$

Intercambiando $\xi_{0}$ por $\xi$ y de la propiedad de simetría de la función de Green, se tiene que la solución al problema de contorno usando la fórmula de Green es

$$
w(\xi)=-\int_{1}^{\xi_{0}} G\left(\xi_{0}, \xi\right) f\left(\xi_{0}\right) d \xi_{0}+p\left[w\left(\xi_{0}\right) \frac{d G\left(\xi, \xi_{0}\right)}{d \xi_{0}}-G\left(\xi, \xi_{0}\right) \frac{d w\left(\xi_{0}\right)}{d \xi_{0}}\right]_{1}^{\xi_{2}}
$$

Reemplazando las condiciones de frontera

$$
w(\xi)=-\int_{1}^{\xi_{p}} G\left(\xi_{0}, \xi\right) f\left(\xi_{0}\right) d \xi_{0}-p \alpha G(\xi, 1)\left[\frac{d w\left(\xi_{0}\right)}{d \xi_{0}}\right]_{1}^{\xi_{c}}
$$

La función de Green puede ser determinada por el siguiente problema de valores a la frontera para cada $\xi_{0}$

$$
\frac{d}{d \xi}\left[p(\xi) \frac{d G_{n}}{d \xi}\right]+q(\xi) G=\delta\left(\xi-\xi_{0}\right)
$$

Sujeto a las siguientes condiciones

$$
\begin{aligned}
& \frac{d G(\xi, 1)}{d \xi}=0 \\
& G\left(\xi, \xi_{e}\right)=0
\end{aligned}
$$




$$
\begin{gathered}
\lim _{\xi \rightarrow \delta_{0^{+}}} G=\lim _{\xi \rightarrow \xi_{\xi^{-}}} G \\
\lim _{\xi \rightarrow \xi_{0^{+}}} \frac{d G(\xi)}{d \xi}-\lim _{\xi \rightarrow \xi_{0^{-}}} \frac{d G(\xi)}{d \xi}=-\frac{1}{p(\xi)}
\end{gathered}
$$

Es decir, la función de Green debe ser continua en $\xi=\xi_{0}$ y su derivada presenta una discontinuidad de salto en este mismo punto.

Suponiendo que la ecuación diferencial ordinaria homogénea asociada del problema de valores a la frontera no-homogéneo tiene como solución combinación lineal de las funciones $v_{1}$ y $v_{2}$ que son linealmente independientes, entonces la función de Green para dicho problema es

$$
\begin{aligned}
& G\left(\xi, \xi_{0}\right)=\left\{\begin{array}{r}
\frac{1}{K D}\left[v_{1}^{\prime}(1) v_{2}\left(\xi_{0}\right)-v_{2}^{\prime}(1) v_{1}\left(\xi_{0}\right)\right]\left[v_{1}(\xi) v_{2}\left(\xi_{e}\right)-v_{2}(\xi) v_{1}\left(\xi_{e}\right)\right] \\
\frac{1}{K D}\left[v_{1}^{\prime}(1) v_{2}(\xi)-v_{2}^{\prime}(1) v_{1}(\xi)\right]\left[v_{1}\left(\xi_{0}\right) v_{2}\left(\xi_{e}\right)-v_{2}\left(\xi_{0}\right) v_{1}\left(\xi_{e}\right)\right] \\
\xi \leq \xi_{0}
\end{array}\right. \\
& K \equiv p(\xi)\left[v_{1}^{\prime}(\xi) v_{2}(\xi)-v_{2}^{\prime}(\xi) v_{1}(\xi)\right]=c t e \\
& D=v_{1}^{\prime}(1) v_{2}\left(\xi_{e}\right)-v_{2}^{\prime}(1) v_{1}\left(\xi_{e}\right) \neq 0
\end{aligned}
$$




\section{A.4Construcción de la función de Green para la ecuación diferencial ordinaria de segundo grado con condiciones de frontera Neumann- Dirichlet}

Equation Chapter 1 Section 4

Consideremos la ecuación diferencial ordinaria de segundo orden definida en el intervalo $\left[1, \xi_{e}\right]$, escrita en su forma auto-adjunta

$$
\frac{d}{d \xi}\left(p(\xi) \frac{d a}{d \xi}\right)+q(\xi) a=-f
$$

Sujeta a las condiciones de frontera

$$
\begin{aligned}
& a^{\prime}(1)=\alpha \\
& a\left(\xi_{e}\right)=0
\end{aligned}
$$

Consideremos ahora la función

$$
w(\xi)=-v_{1}(\xi) \int_{1}^{\xi} v_{2}\left(\xi_{0}\right) f\left(\xi_{0}\right) d \xi_{0}+v_{2}(\xi) \int_{1}^{\xi} v_{1}\left(\xi_{0}\right) f\left(\xi_{0}\right) d \xi_{0}
$$

Realizando la siguiente derivación

$$
\begin{aligned}
\frac{d}{d \xi}\left[p(\xi) \frac{d w}{d \xi}\right] & =-\frac{d}{d \xi}\left[p(\xi) \frac{d v_{1}}{d \xi}\right] \int_{1}^{\xi} v_{2}\left(\xi_{0}\right) f\left(\xi_{0}\right) d \xi_{0} \\
& +\frac{d}{d \xi}\left[p(\xi) \frac{d v_{2}}{d \xi}\right] \int_{1}^{\xi} v_{1}\left(\xi_{0}\right) f\left(\xi_{0}\right) d \xi_{0} \\
& -p(\xi)\left[v_{1}^{\prime}(\xi) v_{2}(\xi)-v_{2}^{\prime}(\xi) v_{1}(\xi)\right] f(\xi)
\end{aligned}
$$

Como $v_{1}$ y $v_{2}$ son soluciones a la ecuación homogénea asociada, entonces $\frac{d}{d \xi}\left[p(\xi) \frac{d v_{i}}{d \xi}\right]=-q(\xi) v_{i}(\xi), i=1,2$, de esta manera se tiene que 


$$
\frac{d}{d \xi}\left[p(\xi) \frac{d w}{d \xi}\right]=-q(\xi) w(\xi)-p(\xi)\left[v_{1}^{\prime}(\xi) v_{2}(\xi)-v_{2}^{\prime}(\xi) v_{1}(\xi)\right] f(\xi)
$$

Por otro lado,

$$
\begin{aligned}
\frac{d}{d \xi}\left\{p\left[v_{1}^{\prime} v_{2}-v_{2}^{\prime} v_{1}\right]\right\} & =\frac{d}{d \xi}\left(p v_{1}^{\prime}\right) v_{2}-\frac{d}{d \xi}\left(p v_{2}^{\prime}\right) v_{1} \\
& +p v_{1}^{\prime} v_{2}^{\prime}-p v_{1}^{\prime} v_{2}^{\prime} \\
& =-q v_{1} v_{2}+q v_{1} v_{2} \\
& =0
\end{aligned}
$$

Del resultado anterior, se concluye que la siguiente expresión es constante, esto es

$$
p(\xi)\left[v_{1}^{\prime}(\xi) v_{2}(\xi)-v_{2}^{\prime}(\xi) v_{1}(\xi)\right] \cong K
$$

Se ha entonces demostrado que la función $w$ satisface la ecuación diferencial ordinaria

$$
\frac{d}{d \xi}\left[p(\xi) \frac{d w}{d \xi}\right]+q(\xi) w(\xi)=-K f(\xi)
$$

Dividiendo por la constante $K$,

$$
\frac{d}{d \xi}\left[p(\xi) \frac{d \frac{w}{K}}{d \xi}\right]+q(\xi) \frac{w(\xi)}{K}=-f(\xi)
$$

Así, la solución general al problema de valores a la frontera es 


$$
a(\xi)=-\int_{1}^{\xi} H\left(\xi, \xi_{0}\right) f\left(\xi_{0}\right) d \xi_{0}+c_{1} v_{1}(\xi)+c_{2} v_{2}(\xi)
$$

Donde

$$
\begin{aligned}
H\left(\xi, \xi_{0}\right) & =\frac{v_{1}(\xi) v_{2}\left(\xi_{0}\right)-v_{2}(\xi) v_{1}\left(\xi_{0}\right)}{p(\xi)\left[v_{1}^{\prime}(\xi) v_{2}(\xi)-v_{2}^{\prime}(\xi) v_{1}(\xi)\right]} \\
& =\frac{1}{K}\left[v_{1}(\xi) v_{2}\left(\xi_{0}\right)-v_{2}(\xi) v_{1}\left(\xi_{0}\right)\right]
\end{aligned}
$$

Derivando la función $H$,

$$
\frac{d}{d \xi} H\left(\xi, \xi_{0}\right)=\frac{1}{K}\left[v_{1}^{\prime}(\xi) v_{2}\left(\xi_{0}\right)-v_{2}^{\prime}(\xi) v_{1}\left(\xi_{0}\right)\right]
$$

Para poder determinar las constantes, es necesario hacer uso de las condiciones de frontera,

Usando la regla de Leibniz se tiene que la derivada de la función $a$ es

$$
a^{\prime}(\xi)=-\int_{1}^{\xi} \frac{\partial H\left(\xi, \xi_{0}\right)}{\partial \xi} f\left(\xi_{0}\right) d \xi_{0}+H\left(1, \xi_{0}\right) f\left(\xi_{0}\right) \frac{d x^{0}}{\not \partial \xi}+H(\xi, \xi) f(\xi) \frac{d \xi}{d \xi}+c_{1} v_{1}^{\prime}(\xi)+c_{2} v_{2}^{\prime}(\xi)
$$

De donde

$$
\begin{gathered}
a^{\prime}(\xi)=-\int_{1}^{\xi} \frac{\partial H\left(\xi, \xi_{0}\right)}{\partial \xi} f\left(\xi_{0}\right) d \xi_{0}+c_{1} v_{1}^{\prime}(\xi)+c_{2} v_{2}^{\prime}(\xi) \\
\alpha=a^{\prime}(1)=c_{1} v_{1}^{\prime}(1)+c_{2} v_{2}^{\prime}(1)
\end{gathered}
$$

De la condición de frontera en el radio externo,

$$
\int_{1}^{\xi_{e}} H\left(\xi_{e}, \xi_{0}\right) f\left(\xi_{0}\right) d \xi_{0}=c_{1} v_{1}\left(\xi_{e}\right)+c_{2} v_{2}\left(\xi_{e}\right)
$$


El sistema lineal de ecuaciones para determinar a $c_{1}$ y $c_{2}$ tiene solución siempre que el determinante sea distinto de cero, esto es

$$
D=v_{1}^{\prime}(1) v_{2}\left(\xi_{e}\right)-v_{2}^{\prime}(1) v_{1}\left(\xi_{e}\right) \neq 0
$$

Siguiendo el método de Cramer para la solución de sistemas de ecuaciones lineales, se tiene que

$$
\begin{aligned}
& c_{1}=\frac{1}{D}\left\{\alpha v_{2}\left(\xi_{e}\right)-v_{2}^{\prime}(1) \int_{1}^{\xi_{e}} H\left(\xi_{e}, \xi_{0}\right) f\left(\xi_{0}\right) d \xi_{0}\right\} \\
& c_{2}=\frac{1}{D}\left\{v_{1}^{\prime}(1) \int_{1}^{\xi_{e}} H\left(\xi_{e}, \xi_{0}\right) f\left(\xi_{0}\right) d \xi_{0}-\alpha v_{1}\left(\xi_{e}\right)\right\}
\end{aligned}
$$

Sustituyendo los valores de las constantes en la solución general de la ecuación diferencial ordinaria no homogénea,

$$
\begin{aligned}
a(\xi) & =-\int_{1}^{\xi}\left[H\left(\xi, \xi_{0}\right)+\frac{v_{2}^{\prime}(1) v_{1}(\xi)-v_{1}^{\prime}(1) v_{2}(\xi)}{D} H\left(\xi_{e}, \xi_{0}\right)\right] f\left(\xi_{0}\right) d \xi_{0} \\
& -\int_{\xi}^{\xi_{2}}\left[\frac{v_{2}^{\prime}(1) v_{1}(\xi)-v_{1}^{\prime}(1) v_{2}(\xi)}{D} H\left(\xi_{e}, \xi_{0}\right)\right] f\left(\xi_{0}\right) d \xi_{0} \\
& +\alpha \frac{v_{2}\left(\xi_{e}\right) v_{1}(\xi)-v_{1}\left(\xi_{e}\right) v_{2}(\xi)}{D}
\end{aligned}
$$

Después de algunos cálculos encontramos que

$$
\begin{aligned}
H\left(\xi, \xi_{0}\right)+\frac{v_{2}^{\prime}(1) v_{1}(\xi)-v_{1}^{\prime}(1) v_{2}(\xi)}{D} H\left(\xi_{e}, \xi_{0}\right)= & \frac{1}{K D}\left[v_{1}^{\prime}(1) v_{2}\left(\xi_{0}\right)-v_{2}^{\prime}(1) v_{1}\left(\xi_{0}\right)\right] \\
& \times\left[v_{1}(\xi) v_{2}\left(\xi_{e}\right)-v_{2}(\xi) v_{1}\left(\xi_{e}\right)\right]
\end{aligned}
$$




$$
\begin{aligned}
\frac{v_{2}^{\prime}(1) v_{1}(\xi)-v_{1}^{\prime}(1) v_{2}(\xi)}{D} H\left(\xi_{e}, \xi_{0}\right) & =\frac{1}{K D}\left[v_{1}^{\prime}(1) v_{2}(\xi)-v_{2}^{\prime}(1) v_{1}(\xi)\right] \\
& \times\left[v_{1}\left(\xi_{0}\right) v_{2}\left(\xi_{e}\right)-v_{2}\left(\xi_{0}\right) v_{1}\left(\xi_{e}\right)\right] \\
\frac{v_{2}\left(\xi_{e}\right) v_{1}(\xi)-v_{1}\left(\xi_{e}\right) v_{2}(\xi)}{D}= & \frac{p(1)}{K D}\left[v_{1}^{\prime}(1) v_{2}(1)-v_{2}^{\prime}(1) v_{1}(1)\right] \\
& \times\left[v_{1}(\xi) v_{2}\left(\xi_{e}\right)-v_{2}(\xi) v_{1}\left(\xi_{e}\right)\right]
\end{aligned}
$$

Sustituyendo estas dos expresiones en la función $a$,

$$
\begin{array}{r}
a(\xi)=\int_{1}^{\xi} \frac{1}{K D}\left[v_{1}^{\prime}(1) v_{2}\left(\xi_{0}\right)-v_{2}^{\prime}(1) v_{1}\left(\xi_{0}\right)\right]\left[v_{1}(\xi) v_{2}\left(\xi_{e}\right)-v_{2}(\xi) v_{1}\left(\xi_{e}\right)\right] f\left(\xi_{0}\right) d \xi_{0} \\
+\int_{\xi}^{\xi_{e}} \frac{1}{K D}\left[v_{1}^{\prime}(1) v_{2}(\xi)-v_{2}^{\prime}(1) v_{1}(\xi)\right]\left[v_{1}\left(\xi_{0}\right) v_{2}\left(\xi_{e}\right)-v_{2}\left(\xi_{0}\right) v_{1}\left(\xi_{e}\right)\right] f\left(\xi_{0}\right) d \xi_{0} \\
+\alpha \frac{p(1)}{K D}\left[v_{1}^{\prime}(1) v_{2}(1)-v_{2}^{\prime}(1) v_{1}(1)\right]\left[v_{1}(\xi) v_{2}\left(\xi_{e}\right)-v_{2}(\xi) v_{1}\left(\xi_{e}\right)\right]
\end{array}
$$

Definamos la función

$$
G\left(\xi, \xi_{0}\right)=\left\{\begin{array}{rr}
\frac{1}{K D}\left[v_{1}^{\prime}(1) v_{2}\left(\xi_{0}\right)-v_{2}^{\prime}(1) v_{1}\left(\xi_{0}\right)\right]\left[v_{1}(\xi) v_{2}\left(\xi_{e}\right)-v_{2}(\xi) v_{1}\left(\xi_{e}\right)\right] \\
\frac{1}{K D}\left[v_{1}^{\prime}(1) v_{2}(\xi)-v_{2}^{\prime}(1) v_{1}(\xi)\right]\left[v_{1}\left(\xi_{0}\right) v_{2}\left(\xi_{e}\right)-v_{2}\left(\xi_{0}\right) v_{1}\left(\xi_{e}\right)\right] \\
\xi \leq \xi_{0}
\end{array}\right.
$$


La función $G\left(\xi, \xi_{0}\right)$ es la función de Green de cada uno de los problemas de para $a$. Claramente esta función es simétrica, $G\left(\xi, \xi_{0}\right)=G\left(\xi_{0}, \xi\right)$.

Como $\xi \leq \xi_{e}$ entonces para evaluar la función de Green en $\xi=\xi_{e}$, tomamos la segunda función obteniendo

$$
G\left(\xi_{e}, \xi_{0}\right)=0
$$

De manera análoga, como $\xi_{0} \leq \xi_{e}$, entonces de la primer función de la función de Green nos permite obtener

$$
G\left(\xi, \xi_{e}\right)=0
$$

Como $1 \leq \xi$ entonces

$$
G(\xi, 1)=\frac{1}{K D}\left[v_{1}^{\prime}(1) v_{2}(1)-v_{2}^{\prime}(1) v_{1}(1)\right]\left[v_{1}(\xi) v_{2}\left(\xi_{e}\right)-v_{2}(\xi) v_{1}\left(\xi_{e}\right)\right]
$$

Las derivadas parciales de la función de Green son

$$
\frac{\partial}{\partial \xi} G\left(\xi, \xi_{0}\right)=\left\{\begin{array}{rr}
\frac{1}{K D}\left[v_{1}^{\prime}(1) v_{2}\left(\xi_{0}\right)-v_{2}^{\prime}(1) v_{1}\left(\xi_{0}\right)\right]\left[v_{1}^{\prime}(\xi) v_{2}^{\prime}\left(\xi_{e}\right)-v_{2}^{\prime}(\xi)\right. & \left.v_{1}^{\prime}\left(\xi_{e}\right)\right] \\
\frac{1}{K D}\left[v_{1}^{\prime}(1) v_{2}^{\prime}(\xi)-v_{2}^{\prime}(1) v_{1}^{\prime}(\xi)\right]\left[v_{1}\left(\xi_{0}\right) v_{2}\left(\xi_{e}\right)-v_{2}\left(\xi_{0}\right)\right. & \left.v_{1}\left(\xi_{e}\right)\right] \\
\xi \leq \xi_{0}
\end{array}\right.
$$




$$
\frac{\partial}{\partial \xi_{0}} G\left(\xi, \xi_{0}\right)=\left\{\begin{array}{rr}
\frac{1}{K D}\left[v_{1}^{\prime}(1) v_{2}^{\prime}\left(\xi_{0}\right)-v_{2}^{\prime}(1) v_{1}^{\prime}\left(\xi_{0}\right)\right]\left[v_{1}^{\prime}(\xi) v_{2}^{\prime}\left(\xi_{e}\right)-v_{2}^{\prime}(\xi)\right. & \left.v_{1}^{\prime}\left(\xi_{e}\right)\right] \\
\frac{1}{K D}\left[v_{1}^{\prime}(1) v_{2}^{\prime}(\xi)-v_{2}^{\prime}(1) v_{1}^{\prime}(\xi)\right]\left[v_{1}^{\prime}\left(\xi_{0}\right) v_{2}\left(\xi_{e}\right)-v_{2}^{\prime}\left(\xi_{0}\right)\right. & \left.v_{1}\left(\xi_{e}\right)\right] \\
& \xi \leq \xi_{0}
\end{array}\right.
$$

Como $1 \leq \xi$ entonces del primer caso de la función de Green obtenemos

$$
\frac{\partial}{\partial \xi} G(\xi, 1)=0
$$

Mientras que del segundo caso $\left(1 \leq \xi_{0}\right)$, se tiene que

$$
\frac{\partial}{\partial \xi} G\left(1, \xi_{0}\right)=0
$$

La solución en términos de la función de Green es

$$
a(\xi)=\int_{1}^{\xi} G\left(\xi, \xi_{0}\right) f\left(\xi_{0}\right) d \xi_{0}+p(1) \alpha G(\xi, 1)
$$




\section{A.5. Prueba de simetría del problema de cerradura}

\section{Simetría con respecto a la coordenada angular $\theta$}

Equation Chapter 1 Section 5

Definamos la siguiente transformación lineal

$$
\begin{gathered}
L_{\theta}:\left[\xi_{p}, \xi_{e}\right] \times\left[0, \frac{\pi}{2}\right] \times[0,2 \pi] \rightarrow\left[1, \xi_{e}\right] \times\left[\frac{\pi}{2}, \pi\right] \times[0,2 \pi] \\
L_{\theta}(\xi, \theta, \phi)=(\xi, \bar{\theta}, \phi)=(\xi, \pi-\theta, \phi)
\end{gathered}
$$

Sabemos que las funciones trigonométricas cumplen las siguientes identidades

$$
\begin{gathered}
\operatorname{sen} \bar{\theta}=\operatorname{sen}(\pi-\theta)=\operatorname{sen} \theta \\
\cos \bar{\theta}=\cos (\pi-\theta)=-\cos \theta
\end{gathered}
$$

De las identidades (A.5.2)-(A.5.3) y de las ecuaciones (5.3.1), (5.3.2) y (5.3.10) tenemos que

$$
\begin{gathered}
U_{\gamma \xi}(\xi, \theta)=-U_{\gamma \xi^{(}}(\xi, \bar{\theta}) \\
U_{\gamma \theta}(\xi, \theta)=U_{\gamma \theta}(\xi, \bar{\theta}) \\
\tilde{U}_{\gamma \lambda}(\xi, \theta, \phi)=-\tilde{U}_{\gamma \lambda}(\xi, \bar{\theta}, \phi)
\end{gathered}
$$

Aplicando la transformación de coordenadas lineal dada por la ecuación (A.5.1) al problema de valores a la frontera dado por las ecuaciones (6.2.1)-(6.2.6) se tiene que: 


$$
\begin{gathered}
\tilde{U}_{y \lambda}(\xi, \bar{\theta})-\left(U_{r \xi}(\xi, \bar{\theta}) \frac{\partial B_{y \lambda}}{\partial \xi}+U_{\bar{\theta}}(\xi, \bar{\theta}) \frac{1}{\xi} \frac{\partial B_{r \lambda}}{\partial \bar{\theta}}\right)= \\
\frac{1}{P e}\left[\frac{1}{\xi^{2}} \frac{\partial}{\partial \xi}\left(\xi^{2} \frac{\partial B_{y \lambda}}{\partial \xi}\right)+\frac{1}{\xi^{2} \operatorname{sen} \bar{\theta}} \frac{\partial}{\partial \bar{\theta}}\left(\operatorname{sen} \bar{\theta} \frac{\partial B_{y \lambda}}{\partial \bar{\theta}}\right)+\frac{1}{\xi^{2} \operatorname{sen}^{2} \bar{\theta}} \frac{\partial^{2} B_{r \lambda}}{\partial \phi^{2}}\right] \\
(\xi, \bar{\theta}, \phi) \in\left(\xi_{p}, \xi_{e}\right) \times\left(\frac{\pi}{2}, \pi\right) \times(0,2 \pi)
\end{gathered}
$$

Arreglando la ecuación (A.5.7), resulta que

$$
\begin{gathered}
\tilde{U}_{\gamma \lambda}(\xi, \bar{\theta})+U_{\gamma \xi}(\xi, \bar{\theta}) \frac{\partial\left(-B_{r i}\right)}{\partial \xi}+U_{y \bar{\theta}}(\xi, \bar{\theta}) \frac{1}{\xi} \frac{\partial\left(-B_{r \lambda}\right)}{\partial \bar{\theta}}= \\
-\frac{1}{P e}\left[\frac{1}{\xi^{2}} \frac{\partial}{\partial \xi}\left(\xi^{2} \frac{\partial\left(-B_{r \lambda}\right)}{\partial \xi}\right)+\frac{1}{\xi^{2} \operatorname{sen} \bar{\theta}} \frac{\partial}{\partial \theta}\left(\operatorname{sen} \bar{\theta} \frac{\partial\left(-B_{r \lambda}\right)}{\partial \bar{\theta}}\right)+\frac{1}{\xi^{2} \operatorname{sen}^{2} \bar{\theta}} \frac{\partial^{2}\left(-B_{y \lambda}\right)}{\partial \phi^{2}}\right] \\
(\xi, \bar{\theta}, \phi) \in\left(\xi_{p}, \xi_{e}\right) \times\left(\frac{\pi}{2}, \pi\right) \times(0,2 \pi)
\end{gathered}
$$

Por otro lado, las condiciones de frontera del problema de cerradura sujetas a la transformación lineal (A.5.1) resultan estar dadas por

C.F.1

$$
\begin{aligned}
& -\cos \bar{\theta}+\frac{\partial B_{r \lambda}}{\partial \xi}=0 \quad \text { en } \xi=\xi_{p},(\bar{\theta}, \phi) \in\left(\pi, \frac{\pi}{2}\right) \times(0,2 \pi) \\
& \cos \bar{\theta}+\frac{\partial\left(-B_{y \lambda}\right)}{\partial \xi}=0 \quad \text { en } \xi=\xi_{p},(\bar{\theta}, \phi) \in\left(\pi, \frac{\pi}{2}\right) \times(0,2 \pi)
\end{aligned}
$$

C. F. 2

$$
B_{y \lambda}=0 \quad \text { en } \xi=\xi_{e},(\bar{\theta}, \phi) \in\left(\pi, \frac{\pi}{2}\right) \times(0,2 \pi)
$$


Dada la forma de la ecuación (A.5.8) y de las condiciones de frontera (A.5.9) y (A.5.11) se puede concluir que la componente $\lambda$ del vector de cerradura no presenta simetría ni antisimetría alrededor de $\theta=\frac{\pi}{2}$.

\section{Simetría con respecto a la coordenada azimutal $\phi$}

Definamos la siguiente transformación lineal

$$
\begin{gathered}
L_{\phi}:\left[\xi_{p}, \xi_{e}\right] \times[0, \pi] \times[0, \pi] \rightarrow\left[1, \xi_{p}\right] \times[0, \pi] \times[\pi, 2 \pi] \\
L_{\phi}(\xi, \theta, \phi)=(\xi, \theta, \bar{\phi})=(\xi, \theta, 2 \pi-\phi)
\end{gathered}
$$

Las funciones trigonométricas seno y coseno satisfacen las ecuaciones siguientes

$$
\begin{gathered}
\operatorname{sen} \bar{\phi}=\operatorname{sen}(2 \pi-\phi)=-\operatorname{sen} \phi \\
\cos \bar{\phi}=\cos (2 \pi-\phi)=\cos \phi
\end{gathered}
$$

Dado que las que el campo de velocidad es axial-simétrico, y de las expresiones anteriores, se tiene que al aplicar la transformación lineal (A.5.12) a la componente $\lambda$ del campo de desviación espacial de la velocidad

$$
\tilde{U}_{\gamma \lambda}(\xi, \theta, \phi)=\tilde{U}_{p \lambda}(\xi, \theta, \bar{\phi})
$$

Aplicando la transformación de coordenadas lineal dada por la ecuación (A.5.12) al problema de valores a la frontera dado por las ecuaciones (6.2.1)-(6.2.6) se tiene que: 


$$
\begin{gathered}
\tilde{U}_{p \lambda}(\xi, \theta)+U_{r \xi}(\xi, \theta) \frac{\partial B_{r \lambda}}{\partial \xi}+U_{r \theta}(\xi, \theta) \frac{1}{\xi} \frac{\partial B_{r \lambda}}{\partial \theta}= \\
\frac{1}{P e}\left[\frac{1}{\xi^{2}} \frac{\partial}{\partial \xi}\left(\xi^{2} \frac{\partial B_{\gamma \lambda}}{\partial \xi}\right)+\frac{1}{\xi^{2} \operatorname{sen} \theta} \frac{\partial}{\partial \theta}\left(\operatorname{sen} \theta \frac{\partial B_{r \lambda}}{\partial \theta}\right)+\frac{1}{\xi^{2} \operatorname{sen}^{2} \theta} \frac{\partial^{2} B_{\gamma \lambda}}{\partial \bar{\phi}^{2}}\right] \\
(\xi, \theta, \bar{\phi}) \in\left(\xi_{p}, \xi_{e}\right) \times(0, \pi) \times(\pi, 2 \pi)
\end{gathered}
$$

C. F. 1

$$
\cos \theta+\frac{\partial B_{y z}}{\partial \xi}=0 \quad \text { en } \xi=\xi_{p}, \quad(\theta, \bar{\phi}) \in(0, \pi) \times(\pi, 2 \pi)
$$

C.F. 2

$$
B_{r \lambda}=0 \quad \text { en } \xi=\xi_{e},(\theta, \bar{\phi}) \in\left(\pi, \frac{\pi}{2}\right) \times(0,2 \pi)
$$

$\mathrm{Al}$ aplicar la transformación (A.5.12) al problema de valores a la frontera que describe al problema de cerradura se puede concluir que dicho promedio es simétrico con respecto a la componente $\phi$ de las coordenadas esféricas en $\phi=\pi$, por lo que se resolverá en un hemisferio de la esfera.

Esta condición de simetría en $\phi$ además de reducir el dominio del problema de cerradura a un hemisferio de la celda esférica de Chang induce las siguientes condiciones de frontera en $\phi$

$$
\begin{array}{ll}
\frac{\partial B_{r \lambda}}{\partial \phi}=0 & \text { en } \phi=0,(\xi, \theta) \in\left(\xi_{p}, \xi_{e}\right) \times(0, \pi) \\
\frac{\partial B_{r \lambda}}{\partial \phi}=0 & \text { en } \phi=\pi,(\xi, \theta) \in\left(\xi_{p}, \xi_{e}\right) \times(0, \pi)
\end{array}
$$




\section{A.6Ecuaciones del ADI 2-D}

Equation Chapter 1 Section 6

Las ecuaciones discretizadas que definen cada uno de los pasos del método ADI, del problema de cerradura dependiente del tiempo de marcha (6.2.2)-(6.2.6) a resolver son:

Paso I

$$
\begin{aligned}
{\left[\frac{\partial B_{y z}}{\partial \tau}\right]_{l, j}^{p+\frac{1}{2}} } & =\left[\frac{1}{\xi^{2}} \frac{\partial}{\partial \xi}\left(\xi^{2} \frac{\partial B_{y z}}{\partial \xi}\right)-P e U_{\gamma \xi} \frac{\partial B_{y z}}{\partial \xi}-P e \tilde{U}_{y z}\right]_{i, j}^{p+\frac{1}{2}}+ \\
& +\left[\frac{1}{\xi^{2} \operatorname{sen} \theta} \frac{\partial}{\partial \theta}\left(\operatorname{sen} \theta \frac{\partial B_{y z}}{\partial \theta}\right)-P e U_{\gamma \theta} \frac{1}{\xi} \frac{\partial B_{y z}}{\partial \theta}\right]_{i, j}^{p}
\end{aligned}
$$

Paso II

$$
\begin{aligned}
{\left[\frac{\partial B_{y z}}{\partial \tau}\right]_{i, j}^{p+1} } & =\left[\frac{1}{\xi^{2}} \frac{\partial}{\partial \xi}\left(\xi^{2} \frac{\partial B_{\gamma z}}{\partial \xi}\right)-P e U_{\gamma \xi} \frac{\partial B_{\gamma \xi}}{\partial \xi}-P e \tilde{U}_{y z}\right]_{i, j}^{p+\frac{1}{2}}+ \\
& +\left[\frac{1}{\xi^{2} \operatorname{sen} \theta} \frac{\partial}{\partial \theta}\left(\operatorname{sen} \theta \frac{\partial B_{\gamma z}}{\partial \theta}\right)-P e U_{\gamma \theta} \frac{1}{\xi} \frac{\partial B_{y z}}{\partial \theta}\right]_{i, j}^{p+1}
\end{aligned}
$$

De acuerdo a la aproximación en diferencias finitas para la primer derivada dada por la ecuación (6.1.1)se tiene que la discretización de las condiciones de frontera e inicial están dadas por

C.F. 1:

$$
\frac{B_{\lambda 2, j, k}^{p}-B_{\lambda 1, j, k}^{p}}{\Delta \xi}=-\cos \theta_{j} \quad j=2, \ldots, N-1, k=2, \ldots, S-1, p>1
$$

C.F. 2:

$$
\frac{B_{\lambda M, j, k}^{p}-B_{2 M-1, j, k}^{p}}{\Delta \xi}=0 \quad j=2, \ldots, N-1, k=2, \ldots, S-1, p>1
$$


C. F. 3:

$$
\frac{B_{i i, 2, k}^{p}-B_{z i, 1, k}^{p}}{\Delta \theta}=0 \quad i=2, \ldots, M-1, k=2, \ldots, S-1, p>1
$$

C.F. 4:

$$
\frac{B_{\lambda i, N, k}^{p}-B_{Z i, N-1, k}^{p}}{\Delta \theta}=0 \quad i=2, \ldots, M-1, k=2, \ldots, S-1, p>1
$$

C. 1.:

$$
B_{\lambda i, j, k}^{1}=f_{i, j} \quad i=2, \ldots, M-1, j=2, \ldots N-1, k=2, \ldots, S-1
$$

Aplicando las definiciones de las aproximaciones de la primera y segunda derivada en diferencias finitas para la variable temporal y a cada una de las componentes esféricas involucradas, dadas por las ecuaciones (6.1.1), (6.1.3), (6.1.4) y (6.1.5), a las ecuaciones que definen los pasos del método $\mathrm{ADI}-2 \mathrm{D}$ (A.6.1)-(A.6.2), se tiene que

Paso I

$$
\begin{aligned}
& \frac{B_{z i, j}^{p+\frac{1}{2}}-B_{z i, j}^{p}}{\Delta \tau / 2} \cong \frac{1}{(\Delta \xi)^{2}}\left[\left(\frac{\xi_{i+\frac{1}{2}}}{\xi_{i}}\right)^{2}\left(B_{z i+1, j}^{p+\frac{1}{2}}-B_{z i, j}^{p+\frac{1}{2}}\right)-\left(\frac{\xi_{i-\frac{1}{2}}}{\xi_{i}}\right)^{2}\left(B_{z i, j}^{p+\frac{1}{2}}-B_{z i-1, j}^{p+\frac{1}{2}}\right)\right]- \\
& -P e U_{\xi}^{i, j} \frac{\alpha_{1}^{\xi} B_{z i+1, j}^{p+\frac{1}{2}}+\alpha_{2}^{\xi} B_{z i, j}^{p+\frac{1}{2}}+\alpha_{3}^{\xi} B_{z i-1, j}^{p+\frac{1}{2}}}{\alpha_{4}^{\xi} \Delta \xi}-P e \tilde{U}_{z}^{i, j}+ \\
& +\frac{1}{\xi_{i}^{2}(\Delta \theta)^{2}}\left[\frac{\operatorname{sen} \theta_{j+\frac{1}{2}}}{\operatorname{sen} \theta_{j}}\left(B_{z i, j+1}^{p}-B_{z i, j}^{p}\right)-\frac{\operatorname{sen} \theta_{j-\frac{1}{2}}}{\operatorname{sen} \theta_{j}}\left(B_{z i, j}^{p}-B_{z i, j-1}^{p}\right)\right]- \\
& -P e U_{\theta}^{i, j} \frac{\alpha_{1}^{\theta} B_{z i, j+1}^{p}+\alpha_{2}^{\theta} B_{z i, j}^{p}+\alpha_{3}^{\theta} B_{z i, j-1}^{p}}{\xi_{i} \alpha_{4}^{\theta} \Delta \theta}
\end{aligned}
$$

Paso II 


$$
\begin{aligned}
\frac{B_{z i, j}^{p+1}-B_{z i, j}^{p+\frac{1}{2}}}{\Delta \tau / 2} & \cong \frac{1}{(\Delta \xi)^{2}}\left[\left(\frac{\xi_{i+\frac{1}{2}}}{\xi_{i}}\right)^{2}\left(B_{z i+1, j}^{p+\frac{1}{2}}-B_{z i, j}^{p+\frac{1}{2}}\right)-\left(\frac{\xi_{i-\frac{1}{2}}}{\xi_{i}}\right)^{2}\left(B_{z i, j}^{p+\frac{1}{2}}-B_{z i-1, j}^{p+\frac{1}{2}}\right)\right]- \\
& -P e U_{\xi}^{i, j} \frac{\alpha_{1}^{\xi} B_{z i+1, j}^{p+\frac{1}{2}}+\alpha_{2}^{\xi} B_{z i, j}^{p+\frac{1}{2}}+\alpha_{3}^{\xi} B_{z i-1, j}^{p+\frac{1}{2}}}{\alpha_{4}^{\xi} \Delta \xi}-P e \tilde{U}_{z}^{i, j}+ \\
& +\frac{1}{\xi_{i}^{2}(\Delta \theta)^{2}}\left[\frac{\operatorname{sen} \theta}{\operatorname{sen} \theta_{j}}\left(B_{z i, j+1}^{p+1}-B_{z i, j}^{p+1}\right)-\frac{\operatorname{sen} \theta_{j-\frac{1}{2}}}{\operatorname{sen} \theta_{j}}\left(B_{z i, j}^{p+1}-B_{Z i, j-1}^{p+1}\right)\right]- \\
& -P e U_{\theta}^{i, j} \frac{\alpha_{1}^{\theta} B_{z i, j+1}^{p+1}+\alpha_{2}^{\theta} B_{z i, j}^{p+1}+\alpha_{3}^{\theta} B_{Z i, j-1}^{p+1}}{\xi_{i} \alpha_{4}^{\theta} \Delta \theta}
\end{aligned}
$$

Definamos los siguientes parámetros numéricos

$$
\begin{gathered}
r_{1}^{\xi}=\frac{\Delta \tau}{\Delta \xi} \\
r_{2}^{\xi}=\frac{\Delta \tau}{(\Delta \xi)^{2}} \\
r_{1}^{\theta}=\frac{\Delta \tau}{\Delta \theta} \\
r_{2}^{\theta}=\frac{\Delta \tau}{(\Delta \theta)^{2}}
\end{gathered}
$$

Multiplicando las ecuaciones (A.6.8) y (A.6.9) por $\Delta \tau / 2$, factorizando e identificando los parámetros numéricos antes definidos (A.6.10)-(A.6.13), obtenemos 
Paso I

$$
\begin{aligned}
& {\left[-\frac{r_{2}^{\xi}}{2}\left(\frac{\xi_{i-\frac{1}{2}}}{\xi_{i}}\right)^{2}+\frac{r_{1}^{\xi}}{2}\left(\frac{\alpha_{3}^{\xi}}{\alpha_{4}^{\xi}}\right) U_{\xi}^{i, j}\right] B_{Z i-1, j}^{p+\frac{1}{2}}+\left\{1+\frac{r_{2}^{\xi}}{2}\left[\left(\frac{\xi_{i+\frac{1}{2}}}{\xi_{i}}\right)^{2}+\left(\frac{\xi_{i-\frac{1}{2}}}{\xi_{i}}\right)^{2}\right]+P e \frac{r_{1}^{\xi}}{2}\left(\frac{\alpha_{2}^{\xi}}{\alpha_{4}^{\xi}}\right) U_{\xi}^{i, j}\right\} B_{2 i, j}^{p+\frac{1}{2}}+} \\
& +\left[-\frac{r_{2}^{\xi}}{2}\left(\frac{\xi_{i+\frac{1}{2}}}{\xi_{i}}\right)^{2}+\frac{r_{1}^{\xi}}{2}\left(\frac{\alpha_{1}^{\xi}}{\alpha_{4}^{\xi}}\right) U_{\xi}^{i, j}\right] B_{\xi i+1, j}^{p+\frac{1}{2}}=-P e \frac{\Delta \tau}{2} \tilde{U}_{z}^{i, j}+\left[\frac{r_{2}^{\theta}}{2 \xi_{i}^{2}} \frac{\operatorname{sen} \theta_{j-\frac{1}{2}}}{\operatorname{sen} \theta_{j}}-P e \frac{r_{1}^{\theta}}{2 \xi_{i}}\left(\frac{\alpha_{3}^{\theta}}{\alpha_{4}^{\theta}}\right) U_{\theta}^{i, j}\right] B_{Z i, j-1}^{p}+ \\
& +\left\{1-\frac{r_{2}^{\theta}}{2 \xi_{i}^{2}}\left[\frac{\operatorname{sen} \theta_{j+\frac{1}{2}}}{\operatorname{sen} \theta_{j-\frac{1}{2}}} \frac{\operatorname{sen} \theta_{j}}{\operatorname{sen} \theta_{j}}\right]-P e \frac{r_{1}^{\theta}}{2 \xi_{i}}\left(\frac{\alpha_{2}^{\theta}}{\alpha_{4}^{\theta}}\right) U_{\theta}^{i, j}\right\} B_{Z i, j}^{p}+\left[\frac{r_{2}^{\theta}}{2 \xi_{i}^{2}} \frac{\operatorname{sen} \theta_{j+\frac{1}{2}}}{\operatorname{sen} \theta_{j}}-P e \frac{r_{1}^{\theta}}{2 \xi_{i}}\left(\frac{\alpha_{1}^{\theta}}{\alpha_{4}^{\theta}}\right) U_{\theta}^{i, j}\right] B_{Z i, j+1}^{p}
\end{aligned}
$$

Paso II

$$
\begin{aligned}
& {\left[-\frac{r_{2}^{\theta}}{2 \xi_{i}^{2}} \frac{\operatorname{sen} \theta_{j-\frac{1}{2}}}{\operatorname{sen} \theta_{j}}+P e \frac{r_{1}^{\theta}}{2 \xi_{i}}\left(\frac{\alpha_{3}^{\theta}}{\alpha_{4}^{\theta}}\right) U_{\theta}^{i, j}\right] B_{z i, j-1}^{p+1}+\left\{1+\frac{r_{2}^{\theta}}{2 \xi_{i}^{2}}\left(\frac{\operatorname{sen} \theta_{j+\frac{1}{2}}}{\operatorname{sen} \theta_{j}}+\frac{\operatorname{sen} \theta_{j-\frac{1}{2}}}{\operatorname{sen} \theta_{j}}\right)+P e \frac{r_{1}^{\theta}}{2 \xi_{i}}\left(\frac{\alpha_{2}^{\theta}}{\alpha_{4}^{\theta}}\right) U_{\theta}^{i, j}\right\} B_{z i, j}^{p+1}+} \\
& \left.+\left[-\frac{r_{2}^{\theta}}{2 \xi_{i}^{2}} \frac{\operatorname{sen} \theta_{j+\frac{1}{2}}}{\operatorname{sen} \theta_{j}}+P e \frac{r_{1}^{\theta}}{2 \xi_{i}}\left(\frac{\alpha_{1}^{\theta}}{\alpha_{4}^{\theta}}\right) U_{\theta}^{i, j}\right] B_{z i, j+1}^{p+1}=-P e \frac{\Delta \tau}{2} \tilde{U}_{2}^{i, j}+\left[\frac{r_{2}^{\xi}}{2} \frac{\xi_{i-\frac{1}{2}}}{\xi_{i}}\right)^{2}-P e \frac{r_{1}^{\xi}}{2}\left(\frac{\alpha_{3}^{\xi}}{\alpha_{4}^{\xi}}\right) U_{\xi}^{i, j}\right] B_{\xi i-1, j}^{p+\frac{1}{2}}+ \\
& +\left\{1-\frac{r_{2}^{\xi}}{2}\left[\left(\frac{\xi_{i+\frac{1}{2}}}{\xi_{i}}\right)^{2}+\left(\frac{\xi_{i-\frac{1}{2}}}{\xi_{i}}\right)^{2}\right]-P e \frac{r_{1}^{\xi}}{2}\left(\frac{\alpha_{2}^{\xi}}{\alpha_{4}^{\xi}}\right) U_{\xi}^{i, j}\right\} B_{z i, j}^{p+\frac{1}{2}}+\left[\frac{r_{2}^{\xi}}{2}\left(\frac{\xi_{i+\frac{1}{2}}}{\xi_{i}}\right)^{2}-P e \frac{r_{1}^{\xi}}{2}\left(\frac{\alpha_{1}^{\xi}}{\alpha_{4}^{\xi}}\right) U_{\xi}^{i, j}\right] B_{z i+1, j}^{p+\frac{1}{2}}
\end{aligned}
$$

Definamos los siguientes coeficientes 


$$
\begin{gathered}
A_{i, j}^{\xi}=r_{2}^{\xi}\left(\frac{\xi_{i-\frac{1}{2}}}{\xi_{i}}\right)^{2}-r_{1}^{\xi} P e\left(\frac{\alpha_{3}^{\xi}}{\alpha_{4}^{\xi}}\right) U_{\xi}^{i, j} \\
B_{i, j}^{\xi}=r_{2}^{\xi}\left[\left(\frac{\xi_{i+\frac{1}{2}}}{\xi_{i}}\right)^{2}+\left(\frac{\xi_{i-\frac{1}{2}}}{\xi_{i}}\right)^{2}\right]+r_{1}^{\xi} P e\left(\frac{\alpha_{2}^{\xi}}{\alpha_{4}^{\xi}}\right) U_{\xi}^{i, j} \\
C_{i, j}^{\xi}=r_{2}^{\xi}\left(\frac{\xi_{i+\frac{1}{2}}}{\xi_{i}}\right)^{2}-r_{1}^{\xi} P e\left(\frac{\alpha_{1}^{\xi}}{\alpha_{4}^{\xi}}\right) U_{\xi}^{i, j} \\
A_{i, j}^{\theta}=\frac{r_{2}^{\theta}}{\xi_{i}^{2}}\left(\frac{\operatorname{sen} \theta}{\operatorname{sen} \theta_{j}}\right)-\frac{r_{1}^{\theta}}{\xi_{i}} P e\left(\frac{\alpha_{3}^{\theta}}{\alpha_{4}^{\theta}}\right) U_{\theta}^{i, j} \\
B_{i, j}^{\theta}=\frac{r_{2}^{\theta}}{\xi_{i}^{2}}\left[\frac{\operatorname{sen} \theta}{\operatorname{sen} \theta_{j}}+\frac{\operatorname{sen} \theta}{\operatorname{sen} \theta_{j}}\right]+\frac{r_{1}^{\theta}}{\xi_{i}} P e\left(\frac{\alpha_{2}^{\theta}}{\alpha_{4}^{\theta}}\right) U_{\theta}^{i, j} \\
C_{i, j}^{\theta}=\frac{r_{2}^{\theta}}{\xi_{i}^{2}} \frac{\operatorname{sen} \theta}{\operatorname{sen} \theta_{j}}-\frac{r_{1}^{\theta}}{\xi_{i}} P e\left(\frac{\alpha_{1}^{\theta}}{\alpha_{4}^{\theta}}\right) U_{\theta}^{i, j}
\end{gathered}
$$

Identificando los coeficientes (A.6.16)-(A.6.21) en las ecuaciones (A.6.14) y (A.6.15), tenemos que las ecuaciones discretizadas son

Paso I

$$
\begin{array}{r}
-A_{i, j}^{\xi} B_{z i-1, j}^{p+\frac{1}{2}}+\left(1+B b_{i, j}^{\xi}\right) B_{z i, j}^{p+\frac{1}{2}}-C_{i, j}^{\xi} B_{z i+1, j}^{p+\frac{1}{2}}=-P e \frac{\Delta \tau}{2} \tilde{U}_{z}^{i, j}+A_{i, j}^{\theta} B_{z i, j-1}^{p}+\left(P e-B b_{i, j}^{\theta}\right) B_{z i, j}^{p} \\
+C_{i, j}^{\theta} B_{z i, j+1}^{p}
\end{array}
$$

Paso II 


$$
\begin{array}{r}
-A_{i, j}^{\theta} B_{z i, j-1}^{p+1}+\left(1+B b_{i, j}^{\theta}\right) B_{z i, j}^{p+1}-C_{i, j}^{\theta} B_{z i, j+1}^{p+1}=-P e \frac{\Delta \tau}{2} \tilde{U}_{z}^{i, j}+A_{i, j}^{\xi} B_{z i-1, j}^{p+\frac{1}{2}}+\left(1-B b_{i, j}^{\xi}\right) B_{z i, j}^{p+\frac{1}{2}} \\
+C_{i, j}^{\xi} B_{2 i+1, j}^{p+\frac{1}{2}}
\end{array}
$$

Paso I

Para cada valor de $j$ y $p$, la ecuación del paso I (A.6.22) representa un sistema tridiagonal de ecuaciones lineales para los valores de $B_{z i-1, j}^{p+\frac{1}{2}}, B_{z i, j}^{p+\frac{1}{2}}$ y $B_{z i+1, j}^{p+\frac{1}{2}}$.

Las ecuaciones del paso I para cada nodo son las siguientes:

Para el nodo $i=2, j=1,2, \ldots, M$ y $p=1,2, \ldots$ y de la condición de frontera (6.2.9.), tenemos

$$
\begin{array}{r}
\left(1-A_{2, j}^{\xi}+B b_{2, j}^{\xi}\right) B_{22, j}^{p+\frac{1}{2}}-C_{i, j}^{\xi} B_{z 3, j}^{p+\frac{1}{2}}=-P e \frac{\Delta \tau}{2} \tilde{U}_{\xi}^{2, j}+A_{2, j}^{\theta} B_{z 2, j-1}^{p}+\left(1-B b_{2, j}^{\theta}\right) B_{z 2, j}^{p} \\
+C_{i, j}^{\theta} B_{22, j+1}^{p}+A_{2, j}^{\xi} \Delta \xi \cos \theta_{j}
\end{array}
$$

Para los nodos $i=3,4, \ldots, N-2, j=1,2, \ldots, M$ y $p=1,2, \ldots$

$$
\begin{array}{r}
-A_{i, j}^{\xi} B_{z i-1, j}^{p+\frac{1}{2}}+\left(1+B b_{i, j}^{\xi}\right) B_{z i, j}^{p+\frac{1}{2}}-C_{i, j}^{\xi} B_{z i+1, j}^{p+\frac{1}{2}}=-P e \frac{\Delta \tau}{2} \tilde{U}_{z}^{i, j}+A_{i, j}^{\theta} B_{z i, j-1}^{p}+\left(1-B b_{i, j}^{\theta}\right) B_{z i, j}^{p} \\
+C_{i, j}^{\theta} B_{z i, j+1}^{p}
\end{array}
$$

Para el nodo $i=N-1, j=1,2, \ldots, M$ y $p=1,2, \ldots$ y de la condición de frontera (6.2.10) obtenemos que 


$$
\begin{array}{r}
-A_{N-1, j}^{\xi} B_{Z N-2, j}^{p+\frac{1}{2}}+\left(1+B b_{N-1, j}^{\xi}-C_{N-1, j}^{\xi}\right) B_{Z N-1, j}^{p+\frac{1}{2}}=-P e \frac{\Delta \tau}{2} \tilde{U}_{z-1, j}^{N-j}+A_{N-1, j}^{\theta} B_{Z N-1, j-1}^{p}+\left(1-B b_{N-1, j}^{\theta}\right) B_{Z N-1, j}^{p} \\
+C_{N-1, j}^{\theta} B_{Z N-1, j+1}^{p}
\end{array}
$$

Paso II

Para cada valor de $i$ y $p$, la ecuación del paso II (A.6.23) representa un sistema tridiagonal de ecuaciones lineales para los valores de $B_{z i, j-1}^{p+1}, B_{z i, j}^{p+1}$ y $B_{z i, j+1}^{p+1}$.

Las ecuaciones del paso II para cada nodo son las siguientes:

Para el nodo $j=2, i=1,2, \ldots, N$ y $p=1,2, \ldots$ y de la condición de frontera (6.2.11) se tiene que

$$
\begin{array}{r}
\left(1+B b_{i, 2}^{\theta}-A_{i, 2}^{\theta}\right) B_{z i, 2}^{p+1}-C_{i, 2}^{\theta} B_{Z i, 3}^{p+1}=-P e \frac{\Delta \tau}{2} \tilde{U}_{2}^{i, 2}+A_{i, 2}^{\xi} B_{Z i-1,2}^{p+\frac{1}{2}}+\left(1-B b_{i, j}^{\xi}\right) B_{Z i, 2}^{p+\frac{1}{2}} \\
+C_{i, j}^{\xi} B_{z i+1,2}^{p+\frac{1}{2}}
\end{array}
$$

Para los nodos $i=1,2, \ldots, N-2, j=3,4, \ldots, M$ y $p=1,2, \ldots$

$$
\begin{array}{r}
-A_{i, j}^{\theta} B_{z i, j-1}^{p+1}+\left(1+B b_{i, j}^{\theta}\right) B_{z i, j}^{p+1}-C_{i, j}^{\theta} B_{z i, j+1}^{p+1}=-P e \frac{\Delta \tau}{2} \tilde{U}_{z}^{i, j}+A_{i, j}^{\xi} B_{z i-1, j}^{p+\frac{1}{2}}+\left(1-B b_{i, j}^{\xi}\right) B_{z i, j}^{p+\frac{1}{2}} \\
+C_{i, j}^{\xi} B_{z i+1, j}^{p+\frac{1}{2}}
\end{array}
$$

Para el nodo $j=M-1, i=1,2, \ldots, N$ y $p=1,2, \ldots$ y de la condición de frontera (6.2.12) obtenemos que

$$
\begin{aligned}
&-A_{i, M-1}^{\theta} B_{z i, M-2}^{p+1}+\left(1+B b_{i, M-1}^{\theta}-C_{i, M-1}^{\theta}\right) B_{Z i, M-1}^{p+1}=-P e \frac{\Delta \tau}{2} \tilde{U}_{Z}^{i, M-1}+A_{i, M-1}^{\xi} B_{z i-1, M-1}^{p+\frac{1}{2}} \\
&+\left(1-B b_{i, j}^{\xi}\right) B_{z i, M-1}^{p+\frac{1}{2}}+C_{i, M-1}^{\xi} B_{z i+1, M-1}^{p+\frac{1}{2}}
\end{aligned}
$$




\section{A.7Ecuaciones del ADI 3-D}

Para determinar numéricamente la componente adimensional del vector de cerradura en la dirección $\lambda\left(B_{\lambda}\right)$ en estado estacionario, usaremos el método ADI (Alternating Direction Implicit Method). Este es un método muy conocido para resolver ecuaciones diferenciales parciales, que para el caso de un problema en tres dimensiones resuelve una sucesión de tres problemas unidimensionales, como se muestra en la figura. Este método particiona el tiempo de marcha de $p$ a $p+1$ en tres pasos: de $p$ a $p+\frac{1}{3}$, de $p+\frac{1}{3}$ a $p+\frac{2}{3}$ y de $p+\frac{2}{3}$ a $p+1$. En el paso I, la dirección $\xi$ es implícita basándose en el método de Crank-Nicholson, mientras que las direcciones $\theta$ y $\phi$ son explicitas. Para cada fila de puntos $(\theta, \phi)$ de la malla, hay $N-2$ ecuaciones correspondientes al punto $(\xi, \theta, \phi)$, las otras ecuaciones correspondientes al punto $(\theta, \phi)$ se obtienen de las condiciones de frontera. Como cada punto en la ecuación (A.7.1) está relacionado con tres incógnitas $B_{\lambda i-1, j, k}^{p+\frac{1}{3}}, B_{\lambda i, j, k}^{p+\frac{1}{3}}$ y $B_{\lambda i+1, j, k}^{p+\frac{1}{3}}$, para cada valor de $(\theta, \phi)$ se tiene un sistema lineal de ecuaciones tridiagonal. Para una malla de tamaño $N \times M \times S$ con un número de nodos de $\Lambda=N M S$, la matriz tridiagonal para cada $(\theta, \phi)$ se resuelve mediante el algoritmo de Thomas. Esto mismo aplica para los pasos II y III. De esta manera, el número de operaciones en el método ADI es del orden de \#iteraciones $\times 3 \times \Lambda$.

Siguiendo el método ADI, la ecuación diferencial parcial a discretizar en cada uno de los pasos es

$$
\begin{array}{r}
\frac{\partial B_{\gamma \lambda}}{\partial \tau}=\frac{1}{P e \xi^{2}} \frac{\partial}{\partial \xi}\left(\xi^{2} \frac{\partial B_{\gamma \lambda}}{\partial \xi}\right)-U_{\gamma \xi} \frac{\partial B_{\gamma \lambda}}{\partial \xi}+\frac{1}{P e \xi^{2} \operatorname{sen} \theta} \frac{\partial}{\partial \theta}\left(\operatorname{sen} \theta \frac{\partial B_{\gamma \lambda}}{\partial \theta}\right) \\
-U_{\gamma \theta}(\xi, \theta) \frac{1}{\xi} \frac{\partial B_{\gamma \lambda}}{\partial \theta}(\xi, \theta)+\frac{1}{P e \xi^{2} \operatorname{sen}^{2} \theta} \frac{\partial^{2} B_{\gamma \lambda}}{\partial \phi^{2}}-\tilde{U}_{\gamma \lambda}(\xi, \theta)
\end{array}
$$



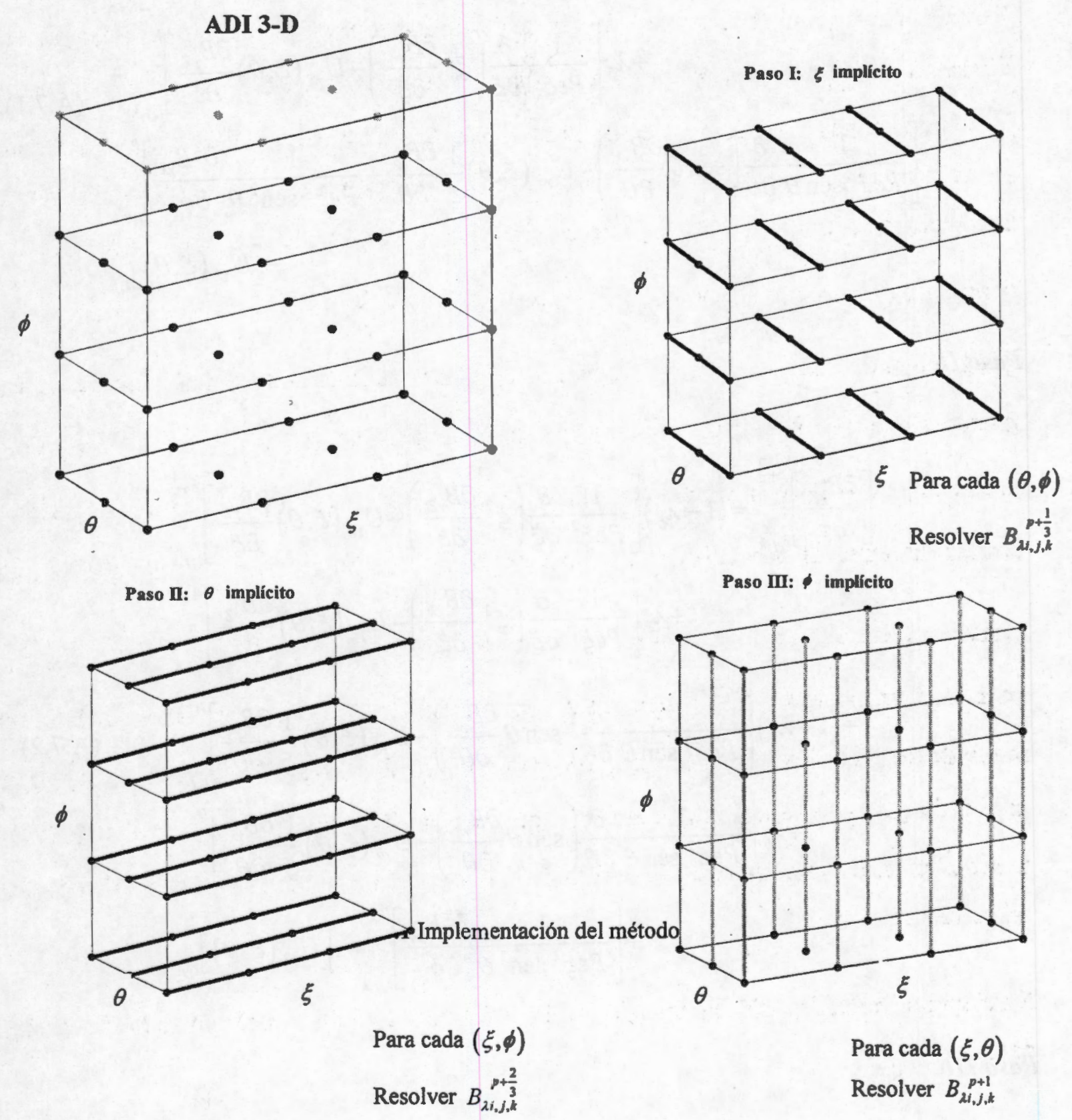

Las ecuaciones discretizadas para cada uno de los pasos son:

Equation Chapter 1 Section 7

Paso I 


$$
\begin{array}{r}
{\left[\frac{\partial B_{\gamma \lambda}}{\partial \tau}\right]_{i, j, k}^{p+\frac{1}{3}}=\left(1-\lambda_{\xi}\right)\left[\frac{1}{P e \xi^{2}} \frac{\partial}{\partial \xi}\left(\xi^{2} \frac{\partial B_{\gamma \lambda}}{\partial \xi}\right)-U_{r \xi}(\xi, \theta) \frac{\partial B_{r \lambda}}{\partial \xi}\right]_{i, j, k}^{p+\frac{1}{3}}} \\
+\lambda_{\xi}\left[\frac{1}{P e \xi^{2}} \frac{\partial}{\partial \xi}\left(\xi^{2} \frac{\partial B_{\gamma z}}{\partial \xi}\right)-U_{r \xi}(\xi, \theta) \frac{\partial B_{r z}}{\partial \xi}\right]_{i, j, k}^{l}+ \\
{\left[\frac{1}{P e \xi^{2} \operatorname{sen} \theta} \frac{\partial}{\partial \theta}\left(\operatorname{sen} \theta \frac{\partial B_{r \lambda}}{\partial \theta}\right)-U_{r \theta}(\xi, \theta) \frac{1}{\xi} \frac{\partial B_{r \lambda}}{\partial \theta}+\frac{1}{P e \xi^{2} \operatorname{sen}^{2} \theta} \frac{\partial^{2} B_{r \lambda}}{\partial \phi^{2}}\right]_{l, j, k}^{p}} \\
-\left[\tilde{U}_{r \lambda}(\xi, \theta)\right]_{i, j}
\end{array}
$$

\section{Paso II}

$$
\begin{array}{r}
{\left[\frac{\partial B_{\gamma z}}{\partial \tau}\right]_{i, j, k}^{p+\frac{2}{3}}=\left(1-\lambda_{\xi}\right)\left[\frac{1}{P e \xi^{2}} \frac{\partial}{\partial \xi}\left(\xi^{2} \frac{\partial B_{\gamma z}}{\partial \xi}\right)-U_{r \xi}(\xi, \theta) \frac{\partial B_{y z}}{\partial \xi}\right]_{i, j, k}^{p+\frac{1}{3}}} \\
+\lambda_{\xi}\left[\frac{1}{P e \xi^{2}} \frac{\partial}{\partial \xi}\left(\xi^{2} \frac{\partial B_{\gamma \lambda}}{\partial \xi}\right)-U_{\gamma \xi}(\xi, \theta) \frac{\partial B_{\gamma z}}{\partial \xi}\right]_{i, j, k}^{p} \\
+\left(1-\lambda_{\theta}\right)\left[\frac{1}{P e \xi^{2} \operatorname{sen} \theta} \frac{\partial}{\partial \theta}\left(\operatorname{sen} \theta \frac{\partial B_{r \lambda}}{\partial \theta}\right)-U_{\gamma \theta}(\xi, \theta) \frac{1}{\xi} \frac{\partial B_{\gamma \lambda}}{\partial \theta}\right]_{i, j, k}^{p+\frac{2}{3}} \\
+\lambda_{\theta}\left[\frac{1}{P e \xi^{2} \operatorname{sen} \theta} \frac{\partial}{\partial \theta}\left(\operatorname{sen} \theta \frac{\partial B_{\gamma \lambda}}{\partial \theta}\right)-U_{\gamma \theta}(\xi, \theta) \frac{1}{\xi} \frac{\partial B_{\gamma \lambda}}{\partial \theta}\right]_{i, j, k}^{p} \\
+\left[\frac{1}{P e \xi^{2} \operatorname{sen}^{2} \theta} \frac{\partial^{2} B_{r \lambda}}{\partial \phi^{2}}\right]_{i, j, k}^{p}-\left[\tilde{U}_{y \lambda}(\xi, \theta)\right]_{i, j}
\end{array}
$$

\section{Paso III}




$$
\begin{aligned}
& {\left[\frac{\partial B_{\gamma \lambda}}{\partial \tau}\right]_{i_{j, j, k}}^{p+1}=\left(1-\lambda_{\xi}\right)\left[\frac{1}{P e \xi^{2}} \frac{\partial}{\partial \xi}\left(\xi^{2} \frac{\partial B_{\gamma \lambda}}{\partial \xi}\right)-U_{r \xi}(\xi, \theta) \frac{\partial B_{\gamma \lambda}}{\partial \xi}\right]_{i_{j}, j, k}^{p+\frac{1}{3}}} \\
& +\lambda_{\xi}\left[\frac{1}{P e \xi^{2}} \frac{\partial}{\partial \xi}\left(\xi^{2} \frac{\partial B_{\gamma \lambda}}{\partial \xi}\right)-U_{\gamma \xi}(\xi, \theta) \frac{\partial B_{\gamma \lambda}}{\partial \xi}\right]_{i, j, k}^{p} \\
& +\left(1-\lambda_{\theta}\right)\left[\frac{1}{P e \xi^{2} \operatorname{sen} \theta} \frac{\partial}{\partial \theta}\left(\operatorname{sen} \theta \frac{\partial B_{\gamma \lambda}}{\partial \theta}\right)-U_{\gamma \theta}(\xi, \theta) \frac{1}{\xi} \frac{\partial B_{\gamma \lambda}}{\partial \theta}\right]_{i, j, k}^{p+\frac{2}{3}} \\
& +\lambda_{\theta}\left[\frac{1}{P e \xi^{2} \operatorname{sen} \theta} \frac{\partial}{\partial \theta}\left(\operatorname{sen} \theta \frac{\partial B_{\gamma \lambda}}{\partial \theta}\right)-U_{\gamma \theta}(\xi, \theta) \frac{1}{\xi} \frac{\partial B_{\gamma \lambda}}{\partial \theta}\right]_{i, j, k}^{p} \\
& +\left(1-\lambda_{\phi}\right)\left[\frac{1}{P e \xi^{2} \operatorname{sen}^{2} \theta} \frac{\partial^{2} B_{y \lambda}}{\partial \phi^{2}}\right]_{i, j, k}^{p+1}+\lambda_{\phi}\left[\frac{1}{P e \xi^{2} \operatorname{sen}^{2} \theta} \frac{\partial^{2} B_{\gamma \lambda}}{\partial \phi^{2}}\right]_{i, j, k}^{p}-\left[\tilde{U}_{p \lambda}(\xi, \theta)\right]_{i, j}
\end{aligned}
$$

Discretización de las condiciones de frontera

De acuerdo a la aproximación en diferencias finitas para la primer derivada dada por la ecuación (6.1.1)se tiene que la discretización de las condiciones de frontera e inicial están dadas por

C.F. 1

$$
\frac{B_{\lambda 2, j, k}^{p}-B_{\lambda 1, j, k}^{p}}{\Delta \xi}=-\cos \theta_{j} \quad j=2, \ldots, N-1, k=2, \ldots, S-1, p>1
$$

C.F. 2

$$
\frac{B_{2 M, j, k}^{p}-B_{2 M-1, j, k}^{p}}{\Delta \xi}=0 \quad j=2, \ldots, N-1, k=2, \ldots, S-1, p>1
$$

C.F. 3

$$
\frac{B_{\lambda i, 2, k}^{p}-B_{z i, 1, k}^{p}}{\Delta \theta}=0 \quad i=2, \ldots, M-1, k=2, \ldots, S-1, p>1
$$


C. F. 4

$$
\frac{B_{\lambda i, N, k}^{p}-B_{z i, N-1, k}^{p}}{\Delta \theta}=0 \quad i=2, \ldots, M-1, k=2, \ldots, S-1, p>1
$$

C. F. 5

$$
\frac{B_{\lambda i, j, 2}^{p}-B_{\lambda i, j, 1}^{p}}{\Delta \phi}=0 \quad i=2, \ldots, M-1, j=2, \ldots, N-1, p>1
$$

C. F. 6

$$
\frac{B_{\lambda i, j, S}^{p}-B_{\lambda i, j, S-1}^{p}}{\Delta \phi}=0 \quad i=2, \ldots, M-1, j=2, \ldots, N-1, p>1
$$

C. I.

$$
B_{\lambda i, j, k}^{1}=f(\xi, \theta, \phi) \quad i=2, \ldots, M-1, j=2, \ldots N-1, k=2, \ldots, S-1
$$

Definamos los siguientes parámetros numéricos

$$
\begin{gathered}
r_{1}^{\xi}=\frac{\Delta \tau}{\Delta \xi} \\
r_{2}^{\xi}=\frac{\Delta \tau}{(\Delta \xi)^{2}} \\
r_{1}^{\theta}=\frac{\Delta \tau}{\Delta \theta} \\
r_{2}^{\theta}=\frac{\Delta \tau}{(\Delta \theta)^{2}} \\
r_{2}^{\phi}=\frac{\Delta \tau}{(\Delta \phi)^{2}}
\end{gathered}
$$

Definamos los siguientes coeficientes 


$$
\begin{gathered}
A_{i, j}^{\xi}=\frac{r_{2}^{\xi}}{P e}\left(\frac{\xi_{i-\frac{1}{2}}}{\xi_{i}}\right)^{2}-r_{1}^{\xi}\left(\frac{\alpha_{3}^{\xi}}{\alpha_{4}^{\xi}}\right) U_{\xi}^{i, j} \\
B_{i, j}^{\xi}=\frac{r_{2}^{\xi}}{P e}\left[\left(\frac{\xi_{i+\frac{1}{2}}}{\xi_{i}}\right)^{2}+\left(\frac{\xi_{i-\frac{1}{2}}}{\xi_{i}}\right)^{2}\right]+r_{1}^{\xi}\left(\frac{\alpha_{2}^{\xi}}{\alpha_{4}^{\xi}}\right) U_{\xi}^{i, j} \\
C_{i, j}^{\xi}=\frac{r_{2}^{\xi}}{P e}\left(\frac{\xi_{i+\frac{1}{2}}}{\xi_{i}}\right)^{2}-r_{1}^{\xi}\left(\frac{\alpha_{1}^{\xi}}{\alpha_{4}^{\xi}}\right) U_{\xi}^{i, j} \\
A_{i, j}^{\theta}=\frac{r_{2}^{\theta}}{P e \xi_{i}^{2}}\left(\frac{\operatorname{sen} \theta_{j-\frac{1}{2}}}{\operatorname{sen} \theta_{j}}\right)_{-\frac{r_{1}^{\theta}}{\xi_{i}}}\left(\frac{\alpha_{3}^{\theta}}{\alpha_{4}^{\theta}}\right) U_{\theta}^{i, j} \\
B_{i, j}^{\theta}=\frac{r_{2}^{\theta}}{P e \xi_{i}^{2}}\left[\frac{\operatorname{sen} \theta}{\operatorname{sen} \theta_{j}}+\frac{\operatorname{sen} \theta_{j-\frac{1}{2}}}{\operatorname{sen} \theta_{j}}\right]+\frac{r_{1}^{\theta}}{\xi_{i}}\left(\frac{\alpha_{2}^{\theta}}{\alpha_{4}^{\theta}}\right) U_{\theta}^{i, j} \\
C_{i, j}^{\theta}=\frac{r_{2}^{\theta}}{P e \xi_{i}^{2}} \frac{\operatorname{sen} \theta_{j+\frac{1}{2}}}{\operatorname{sen} \theta_{j}}-\frac{r_{1}^{\theta}}{\xi_{i}}\left(\frac{\alpha_{1}^{\theta}}{\alpha_{4}^{\theta}}\right) U_{\theta}^{i, j} \\
\operatorname{sen}{ }^{2} \theta_{j}
\end{gathered}
$$

De la aproximación en diferencias finitas de la segunda derivada y de la primera en la dirección $\xi$ dadas por las ecuaciones (6.1.2) y (6.1.4), y de la definición de los coeficientes dados por la ecuaciones (6.2.19)-(6.2.21), definamos

$$
\delta_{\xi}^{p} \equiv\left[\frac{1}{\xi^{2}} \frac{\partial}{\partial \xi}\left(\xi^{2} \frac{\partial B_{\lambda}}{\partial \xi}\right)-P e U_{r \xi}(\xi, \theta) \frac{\partial B_{\lambda}}{\partial \xi}\right]_{i, j, k}^{p}=C_{i, j}^{\xi} B_{\lambda i+1, j, k}^{p}-B_{i, j}^{\xi} B_{\lambda i, j, k}^{p}+A_{i, j}^{\xi} B_{\lambda i-1, j, k}^{p}
$$


Para la dirección en $\theta$, de las expresiones de las derivadas en diferencias finitas para esta dirección, ecuaciones (6.1.2) y (6.1.5), y de las expresiones de los coeficientes obtenidos de la discretización de la ecuación diferencial parcial del problema de cerradura para dicha dirección (6.2.22)-(6.2.24), se puede definir

$$
\delta_{\theta}^{p} \equiv\left[\frac{1}{\xi^{2} \operatorname{sen} \theta} \frac{\partial}{\partial \theta}\left(\operatorname{sen} \theta \frac{\partial B_{\lambda}}{\partial \theta}\right)-P e U_{\gamma \theta}(\xi, \theta) \frac{1}{\xi} \frac{\partial B_{\lambda}}{\partial \theta}\right]_{l, j, k}^{p}=C_{i, j}^{\theta} B_{\lambda i, j+1, k}^{p}-B_{i, j}^{\theta} B_{\lambda i, j, k}^{p}+A_{i, j}^{\theta} B_{\lambda i, j-1, k}^{p}
$$

De la aproximación de la segunda derivada en diferencias finitas para la componente $\phi$ dada por la ecuación (6.1.6) y de la definición del coeficiente dado por la ecuación (6.2.25) se define que

$$
\delta_{\phi}^{p} \equiv\left[\frac{1}{\xi^{2} \operatorname{sen}^{2} \theta} \frac{\partial^{2} B_{\lambda}}{\partial \phi^{2}}\right]_{i, j, k}^{p}=A_{i, j}^{\phi} B_{\lambda i, j, k+1}^{p}-2 A_{i, j}^{\phi} B_{\lambda i, j, k}^{p}+A_{i, j}^{\phi} B_{\lambda i, j, k-1}^{p}
$$

\section{Paso I}

De la condición de frontera dada por la ecuación (6.2.9), se tienè que el paso I para $i=2$

$$
\begin{array}{r}
\left(1+\frac{1-\lambda_{\xi}}{3}\left(B_{2, j}^{\xi}-A_{2, j}^{\xi}\right)\right) B_{\lambda 2, j, k}^{p+\frac{1}{3}}-\frac{1-\lambda_{\xi}}{3} C_{2, j}^{\xi} B_{\lambda 3, j, k}^{p+\frac{1}{3}}=D_{\xi 2, j, k}^{p}+\frac{1-\lambda_{\xi}}{3} A_{2, j}^{\xi} \Delta \xi \cos \theta_{j} \\
j=2, \ldots, N-1, \quad k=2, \ldots, S-1, \quad p>1
\end{array}
$$

$$
\begin{array}{r}
-\frac{1-\lambda_{\xi}}{3} A_{i, j}^{\xi} B_{\lambda i-1, j, k}^{p+\frac{1}{3}}+\left(1+\frac{1-\lambda_{\xi}}{3} B_{i, j}^{\xi}\right) B_{\lambda i, j, k}^{p+\frac{1}{3}}-\frac{1-\lambda_{\xi}}{3} C_{i, j}^{\xi} B_{\lambda i+1, j, k}^{p+\frac{1}{3}}=D_{\xi i, j, k}^{p} \\
i=3, \ldots, M-2, \quad j=2, \ldots, N-1, \quad k=2, \ldots, S-1, \quad p>1
\end{array}
$$


De la condición de frontera dada por la ecuación (6.2.10) resulta que la ecuación del paso I para el nodo $i=M-1$ es

$$
\begin{array}{r}
-\frac{1-\lambda_{\xi}}{3} A_{M-1, j}^{\xi} B_{\lambda M-2, j, k}^{p+\frac{1}{3}}+\left(1+\frac{1-\lambda_{\xi}}{3}\left(B_{M-1, j}^{\xi}-C_{M-1, j}^{\xi}\right)\right) B_{\lambda M-1, j, k}^{p+\frac{1}{3}}=D_{\xi M-1, j, k}^{p} \\
j=2, \ldots, N-1, \quad k=2, \ldots, S-1, \quad p>1
\end{array}
$$

Donde

$$
D_{\xi i, j, k}^{p}=\frac{\lambda_{\xi}}{3} \delta_{\xi}{ }^{p}+B_{\lambda i, j, k}{ }^{p}+\frac{1}{3} \delta_{\theta}{ }^{p}+\frac{1}{3} \delta_{i}{ }^{p}-\frac{\Delta \tau}{3} P e \tilde{U}_{i, j}
$$

El sistema de ecuaciones dado por las expresiones (A.7.26)-(A.7.28) toma la siguiente representación matricial

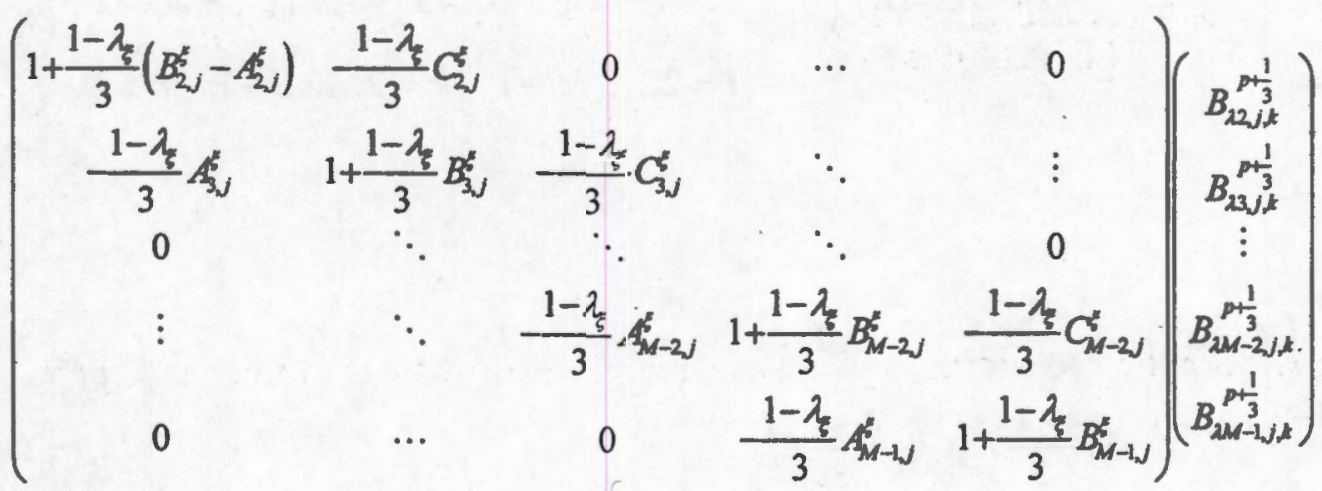

$$
\begin{aligned}
& \left(\begin{array}{c}
D_{\xi 2, j, k}^{p}+\frac{1-\lambda_{\xi}}{3} A_{\Sigma, j}^{p} \Delta \xi \cos \theta_{j} \\
D_{\xi 3, j, k}^{p} \\
\vdots \\
D_{\xi M-2, j, k}^{p} \\
D_{\xi M-1, j,}^{p}
\end{array}\right)
\end{aligned}
$$




\section{Paso II}

$$
\begin{gathered}
\left(1+\frac{1-\lambda_{\theta}}{3}\left(B_{i, 2}^{\theta}-A_{i, 2}^{\theta}\right)\right) B_{\lambda i, 2, k}^{p+\frac{2}{3}}-\frac{1-\lambda_{\theta}}{3} C_{i, 2}^{\theta} B_{\lambda i, 3, k}^{p+\frac{2}{3}}=D_{\theta i, 2, k}^{p+\frac{1}{3}} \\
i=2, \ldots, M-1, \quad k=2, \ldots, S-1, \quad p>1 \\
-\frac{\left(1-\lambda_{\theta}\right)}{3} A_{i, j}^{\theta} B_{2 i, j-1, k}^{1+\frac{2}{3}}+\left(1+\frac{1-\lambda_{\theta}}{3} B_{i, j}^{\theta}\right) B_{\lambda i, j, k}^{p+\frac{2}{3}}-\frac{1-\lambda_{\theta}}{3} C_{i, j}^{\theta} B_{\lambda i, j+1, k}^{p+\frac{2}{3}}=D_{\theta i, j, k}^{p+\frac{1}{3}} \\
i=2, \ldots, M-1, \quad j=3, \ldots, N-2, \quad k=2, \ldots, S-1, \quad p>1
\end{gathered}
$$

Donde

$$
\begin{array}{r}
D_{\theta i, j, k}^{p+\frac{1}{3}}=\frac{1-\lambda_{\xi}}{3} \delta_{\xi}^{p+\frac{1}{3}}+\frac{\lambda_{\xi}}{3} \delta_{\xi}{ }^{p}+\frac{\lambda_{\theta}}{3} \delta_{\theta}{ }^{p}+B_{\lambda i, j, k}^{p}+\frac{1}{3} \delta_{\theta}{ }^{p}-\frac{\Delta \tau}{3} P e \tilde{U}_{i, j} \\
-\frac{\left(1-\lambda_{\theta}\right)}{3} A_{i, N-1}^{\theta} B_{\lambda i, N-2, k}^{p+\frac{2}{3}}+\left(1+\frac{1-\lambda_{\theta}}{3}\left(B_{i, N-1}^{\theta}-C_{i, N-1}^{\theta}\right)\right) B_{\lambda i, N-1, k}^{p+\frac{2}{3}}=D_{\theta i, N-1, k}^{p+\frac{1}{3}} \\
i=2, \ldots, M-1, \quad k=2, \ldots, S-1, \quad p>1
\end{array}
$$

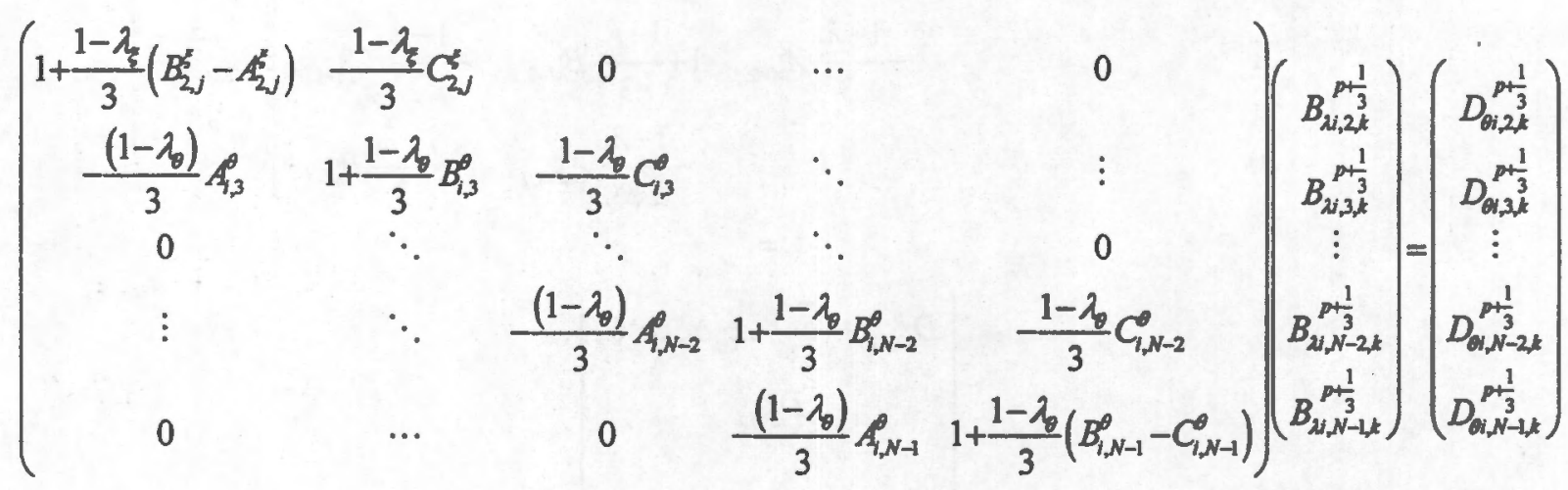

Paso III 


$$
\begin{gathered}
{\left[1+\frac{1}{3}\left(1-\lambda_{\phi}\right) A_{i, j}^{\phi}\right] B_{\lambda i, j, 2}^{p+1}-\frac{1-\lambda_{\phi}}{3} A_{i, j}^{\phi} B_{\lambda i, j, 3}^{p+1}=D_{\phi i, j, 2}^{p+\frac{1}{3}}} \\
i=2, \ldots, M-1, \quad j=2, \ldots, N-1, \quad p>1 \\
-\frac{1-\lambda_{\phi}}{3} A_{i, j}^{\phi} B_{\lambda i, j, k-1}^{p+1}+\left[1+\frac{2}{3}\left(1-\lambda_{\phi}\right) A_{i, j}^{\phi}\right] B_{\lambda i, j, k}^{p+1}-\frac{1-\lambda_{\phi}}{3} A_{i, j}^{\phi} B_{\lambda i, j, k+1}^{p+1}=D_{\phi i, j, k}^{p+\frac{1}{3}} \\
i=2, \ldots, M-1, j=3, \ldots, N-1, \quad k=2, \ldots, S-2, \quad p>1 \\
-\frac{1-\lambda_{\phi}}{3} A_{i, j}^{\phi} B_{\lambda i, j, S-2}^{p+1}+\left[1+\frac{1}{3}\left(1-\lambda_{\phi}\right) A_{i, j}^{\phi}\right] B_{\lambda i, j, S-1}^{p+1}=D_{\phi i, j, S-1}^{p+\frac{1}{3}} \\
i=2, \ldots, M-1, \quad j=2, \ldots, N-1, \quad p>1
\end{gathered}
$$

Donde

$$
D_{\phi i, j, k}^{p+\frac{2}{3}}=\frac{1-\lambda_{\xi}}{3} \delta_{\xi}^{p+\frac{1}{3}}+\frac{\lambda_{\xi}}{3} \delta_{\xi}{ }^{p}+\frac{1-\lambda_{\theta}}{3} \delta_{\theta}^{p+\frac{2}{3}}+\frac{\lambda_{\theta}}{3} \delta_{\theta}{ }^{p}+\frac{\lambda_{\phi}}{3} \delta_{\phi}{ }^{p}+B_{\lambda i, j, k}{ }^{p}-\frac{\Delta \tau}{3} P e \tilde{U}_{i, j}
$$

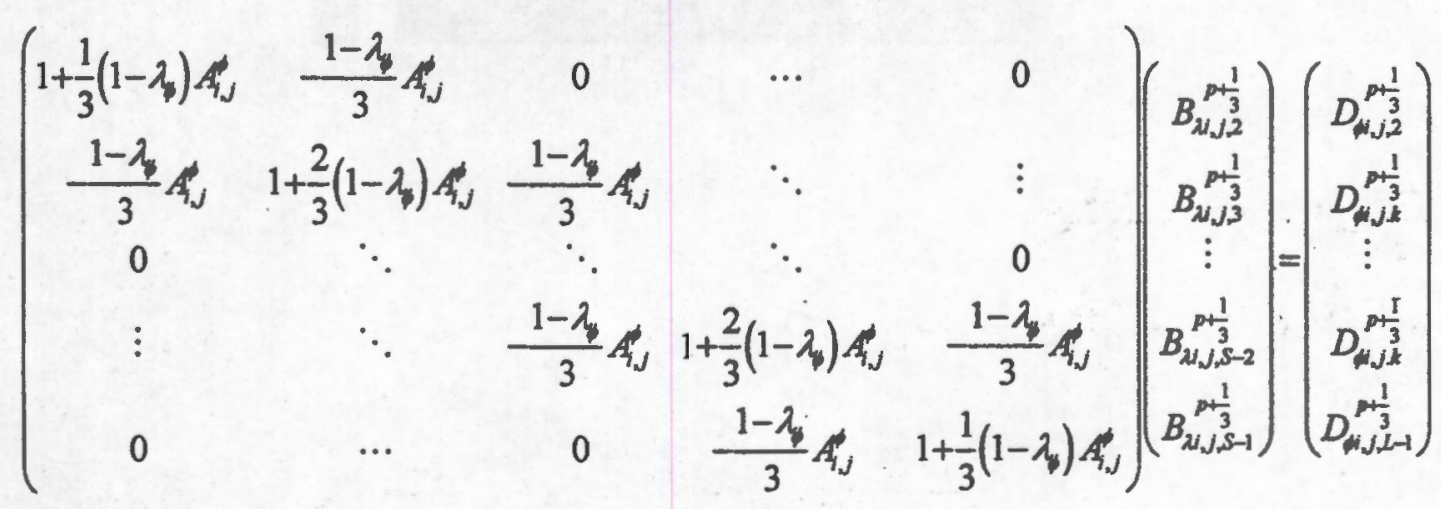




\section{A.8Gráficos de la variable de cerradura}
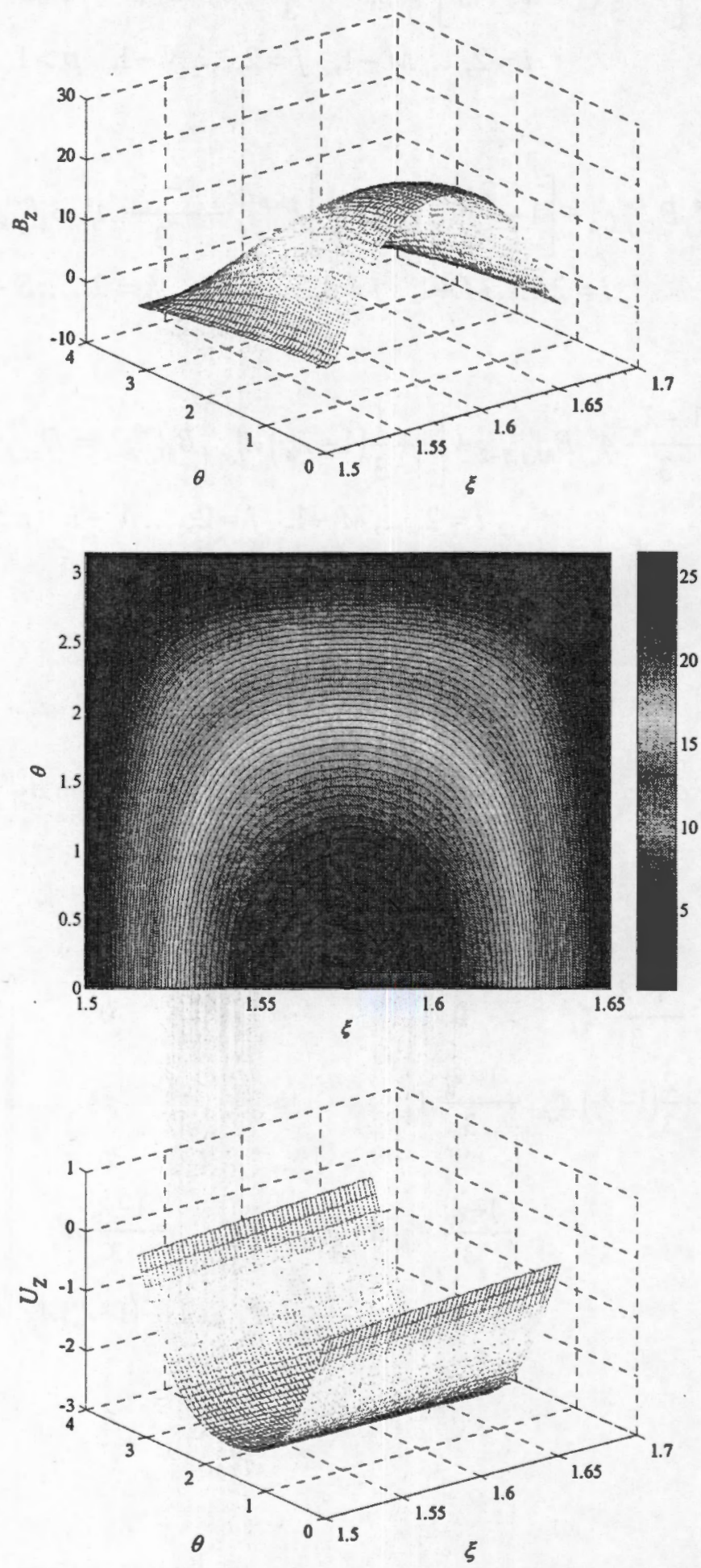

Figura A-7.1: Gráfica de la componente $Z$ del vector de cerradura $\mathbf{B}_{r}$ axialmente simétrico simétrico y de la desviación espacial de la velocidad $\tilde{U}_{z}$; para flujo potencial y porosidad del medio poroso de $\varepsilon_{y}=0.25$ 

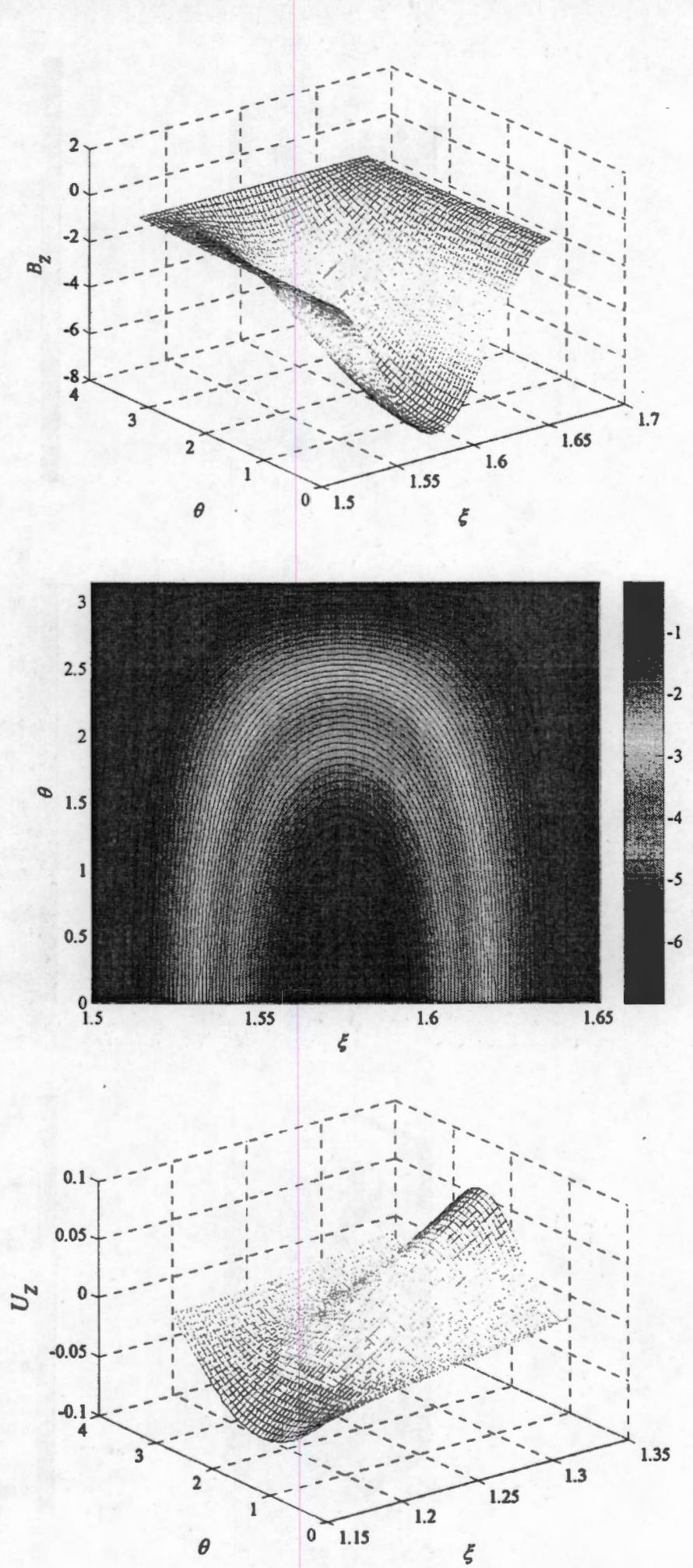

Figura A-7.2: Gráfica de la componente $Z$ del vector de cerradura $\mathbf{B}_{\boldsymbol{y}}$ axialmente simétrico y de la desviación espacial de la velocidad $\tilde{U}_{z}$, para flujo reptante y porosidad del medio poroso de $\varepsilon_{\gamma}=0.30$ 

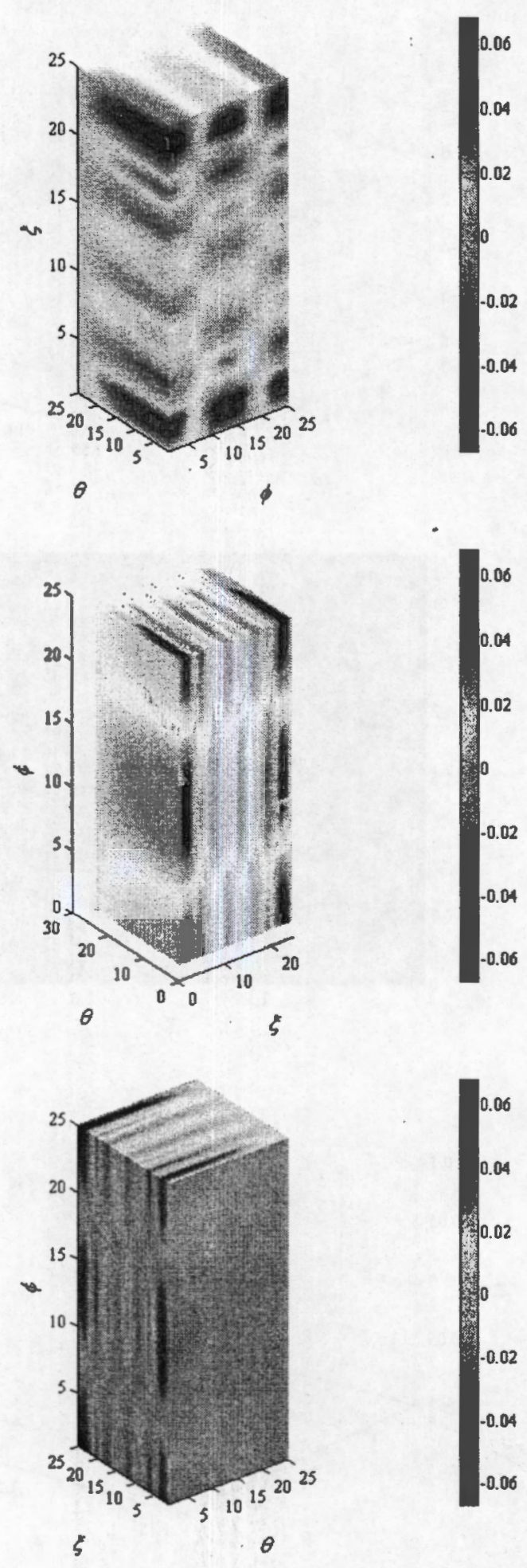

Figura A-7.3: Gráfica de la componente $X$ del vector de cerradura $\mathbf{B}_{r}$ para flujo potencial y porosidad del medio poroso de $\varepsilon_{r}=0.22$ 

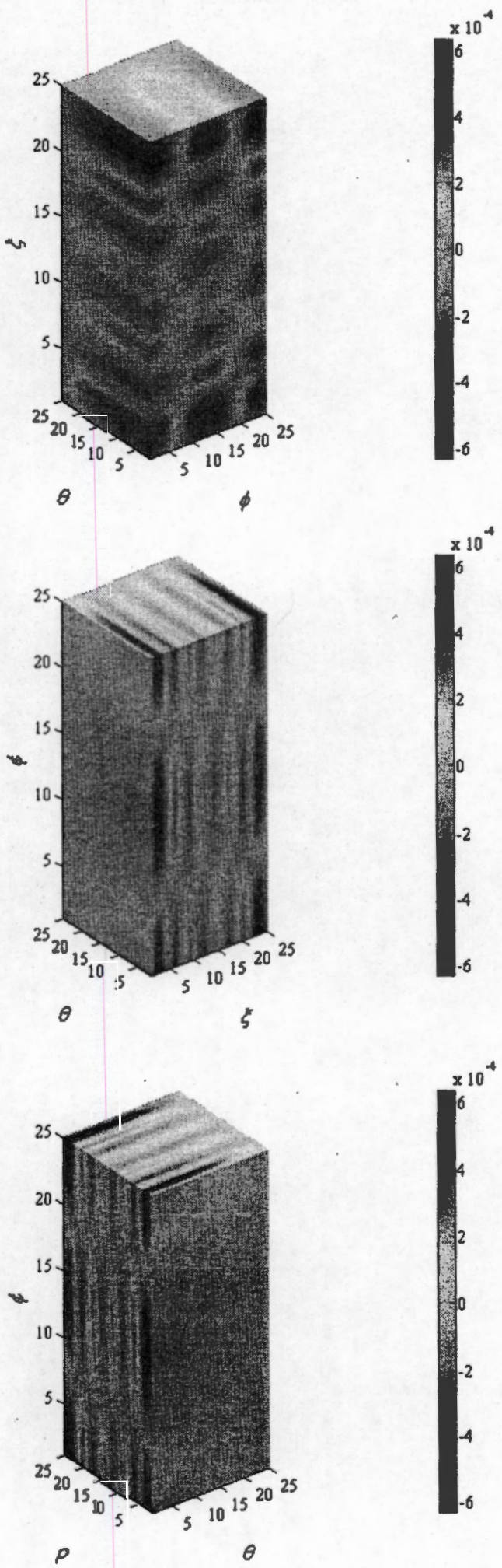

Figura A-7.4: Gráfica de la componente $X$ del vector de cerradura $\mathbf{B}_{y}$ para flujo reptante y porosidad del medio poroso de $\varepsilon_{y}=0.30$ 
